Supporting information

\title{
Discrete Organofluoroaluminate Anions: Synthetic, Structural and Spectroscopic Aspects
}

\author{
Evelin Gruden, ${ }^{\mathrm{a}, \mathrm{b}}$ Melita Tramšek ${ }^{\mathrm{a}}$ and Gašper Tavčar*a,b \\ a Department of Inorganic Chemistry and Technology, Jožef Stefan Institute, Jamova 39, 1000 \\ Ljubljana, Slovenia \\ b Jožef Stefan International Postgraduate School, Jamova 39, 1000 Ljubljana, Slovenia
}

\section{Table of content}

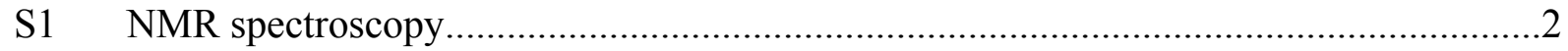

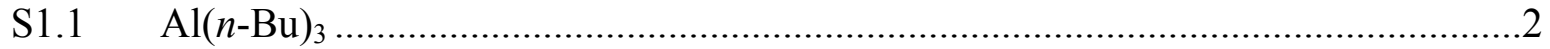

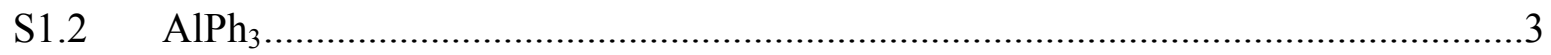

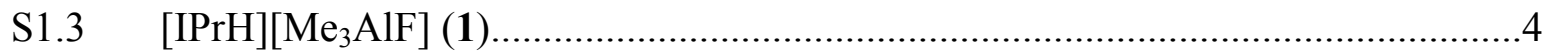

S1.4 Decomposition of $[\mathrm{IPrH}]\left[\mathrm{Me}_{3} \mathrm{AlF}\right](\mathbf{1})$ in acetonitrile.......................................

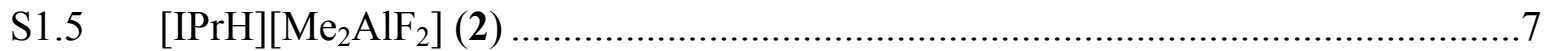

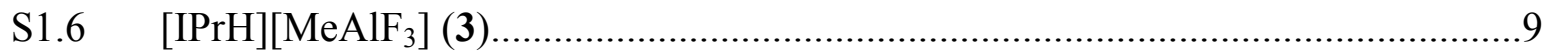

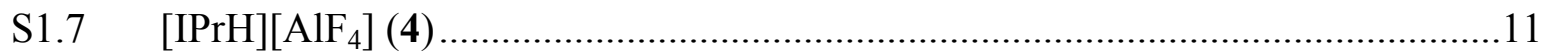

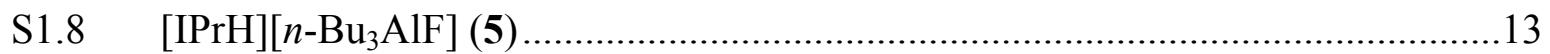

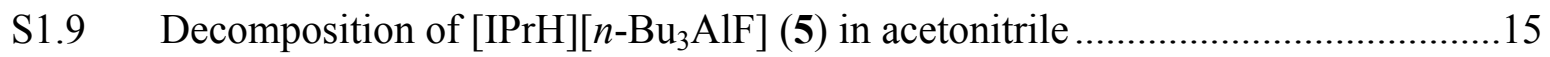

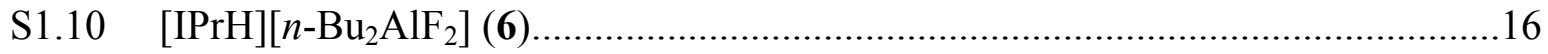

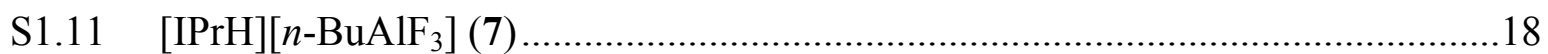

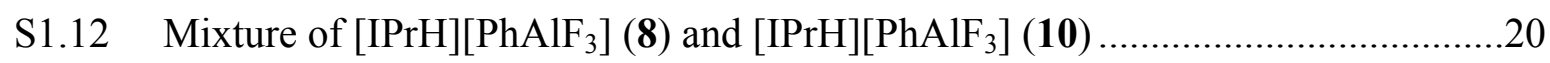

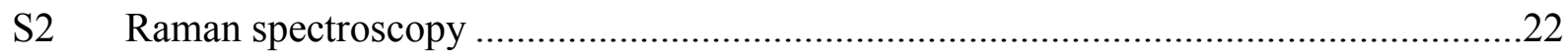

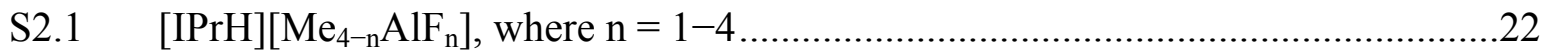

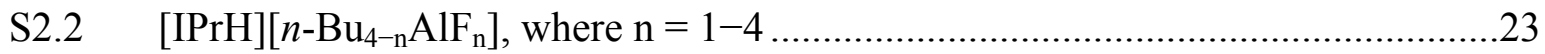

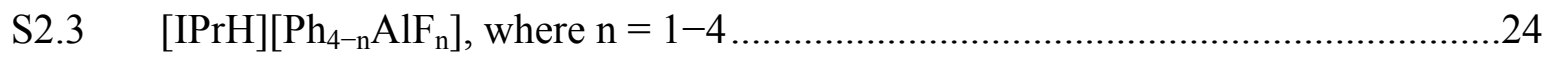

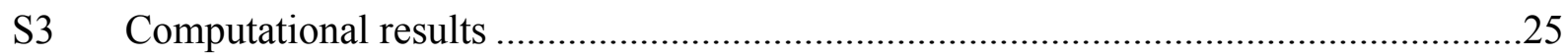

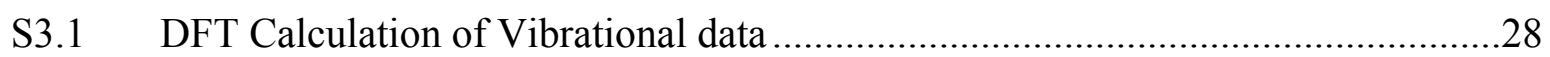

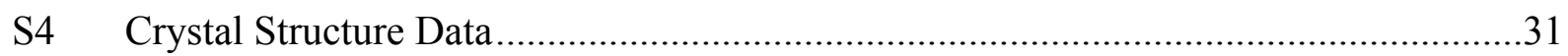

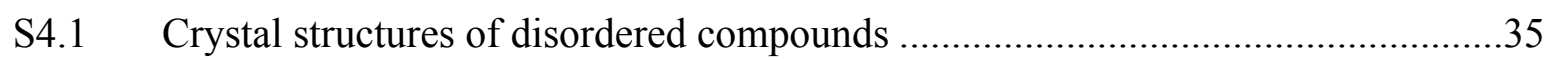




\section{S1 NMR spectroscopy}

\section{$S 1.1 A l(\mathrm{n}-\mathrm{Bu})_{3}$}

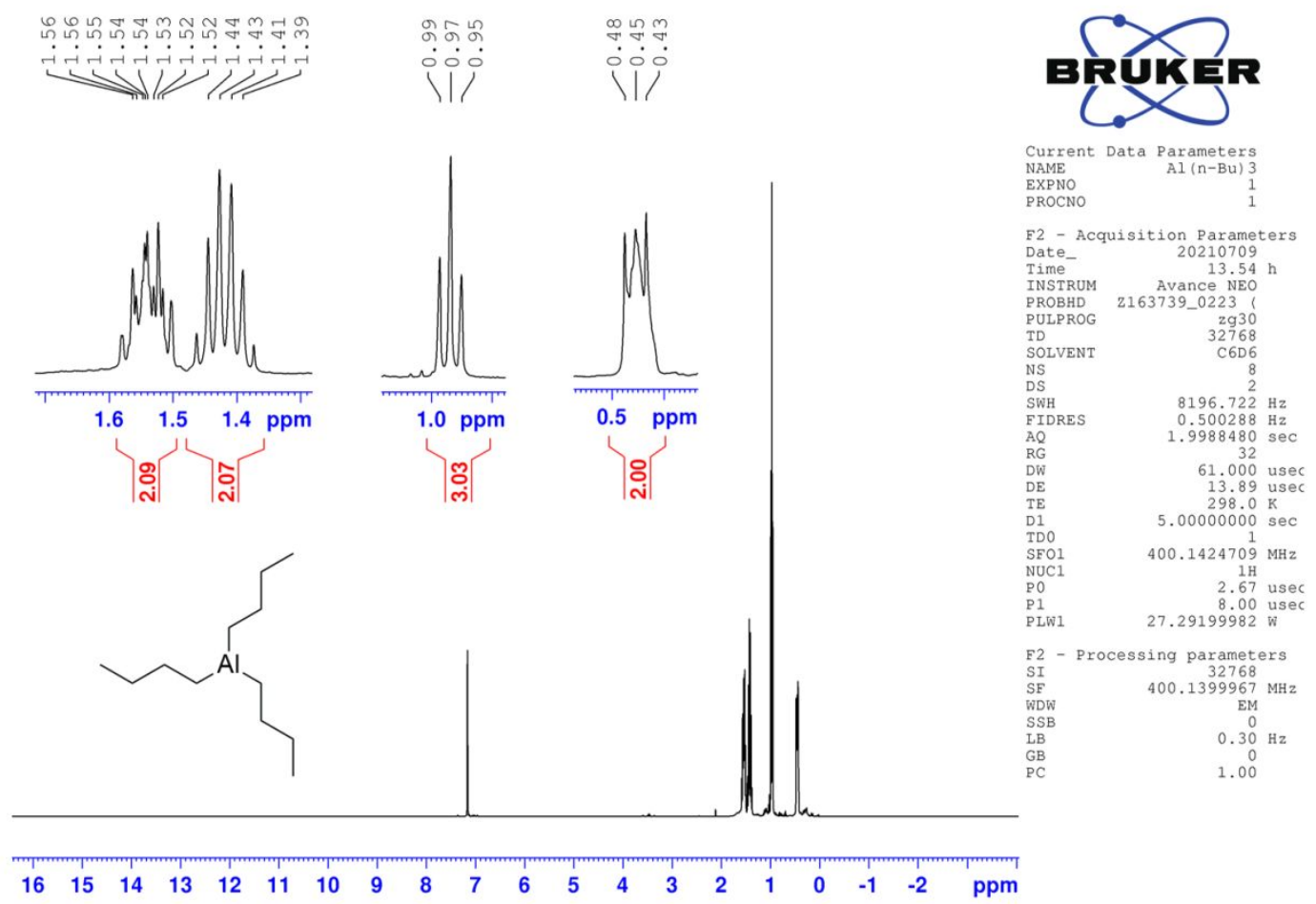

Figure S1. ${ }^{1} \mathrm{H}$ NMR spectrum of $\mathrm{Al}(n-\mathrm{Bu})_{3}$ in $\mathrm{C}_{6} \mathrm{D}_{6}$ solution.

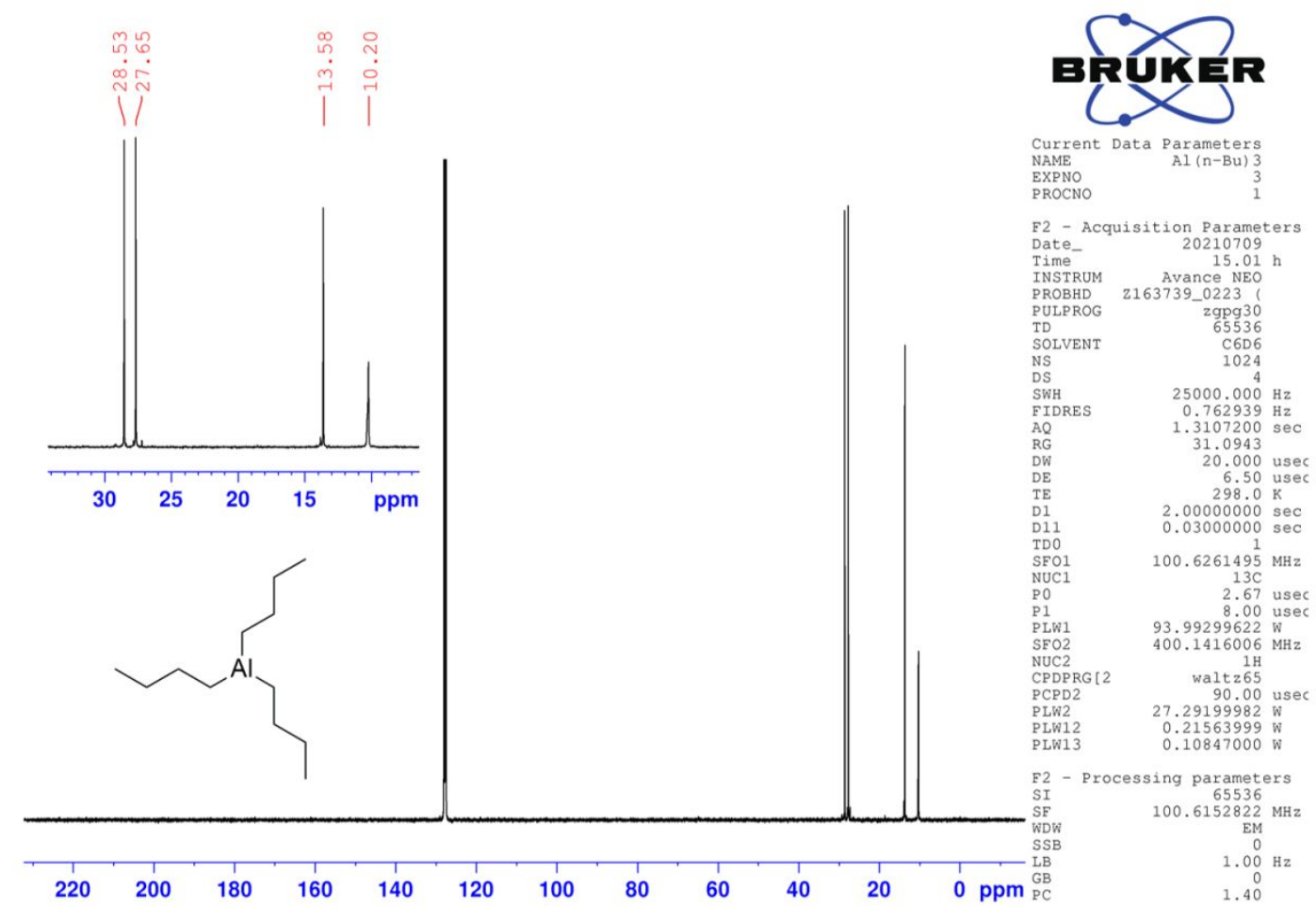

Figure S2. ${ }^{13} \mathrm{C}$ NMR spectrum of $\mathrm{Al}(n-\mathrm{Bu})_{3}$ in $\mathrm{C}_{6} \mathrm{D}_{6}$ solution. 


\section{$\mathrm{S1.2} \mathrm{AlPh}_{3}$}
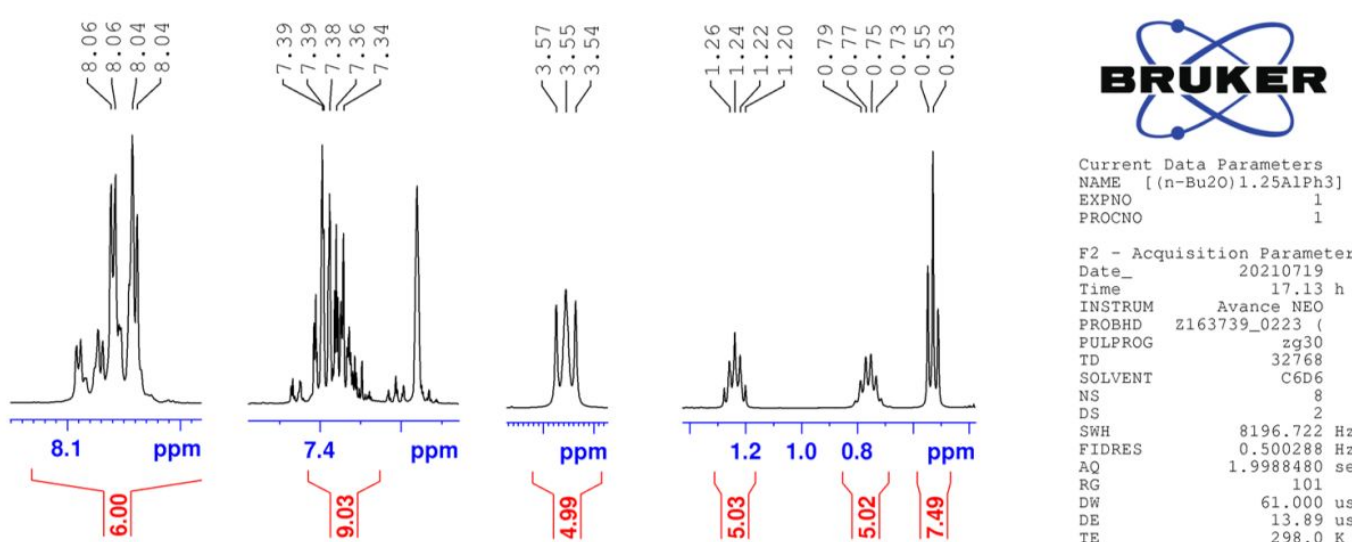

NAME
EXPNO
PROCNO
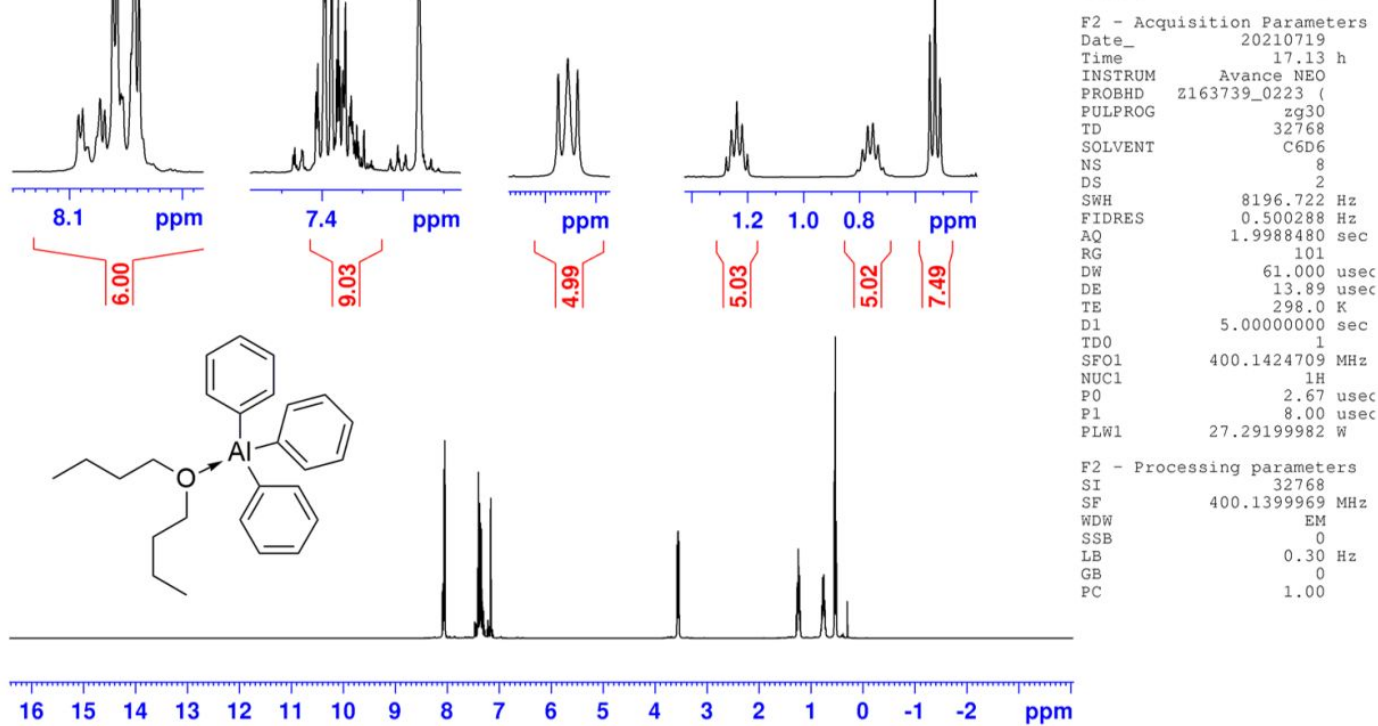

Figure S3. ${ }^{1} \mathrm{H}$ NMR spectrum of $\left[\left(n-\mathrm{Bu}_{2} \mathrm{O}\right)_{1.25} \mathrm{AlPh}_{3}\right]$ in $\mathrm{C}_{6} \mathrm{D}_{6}$ solution.

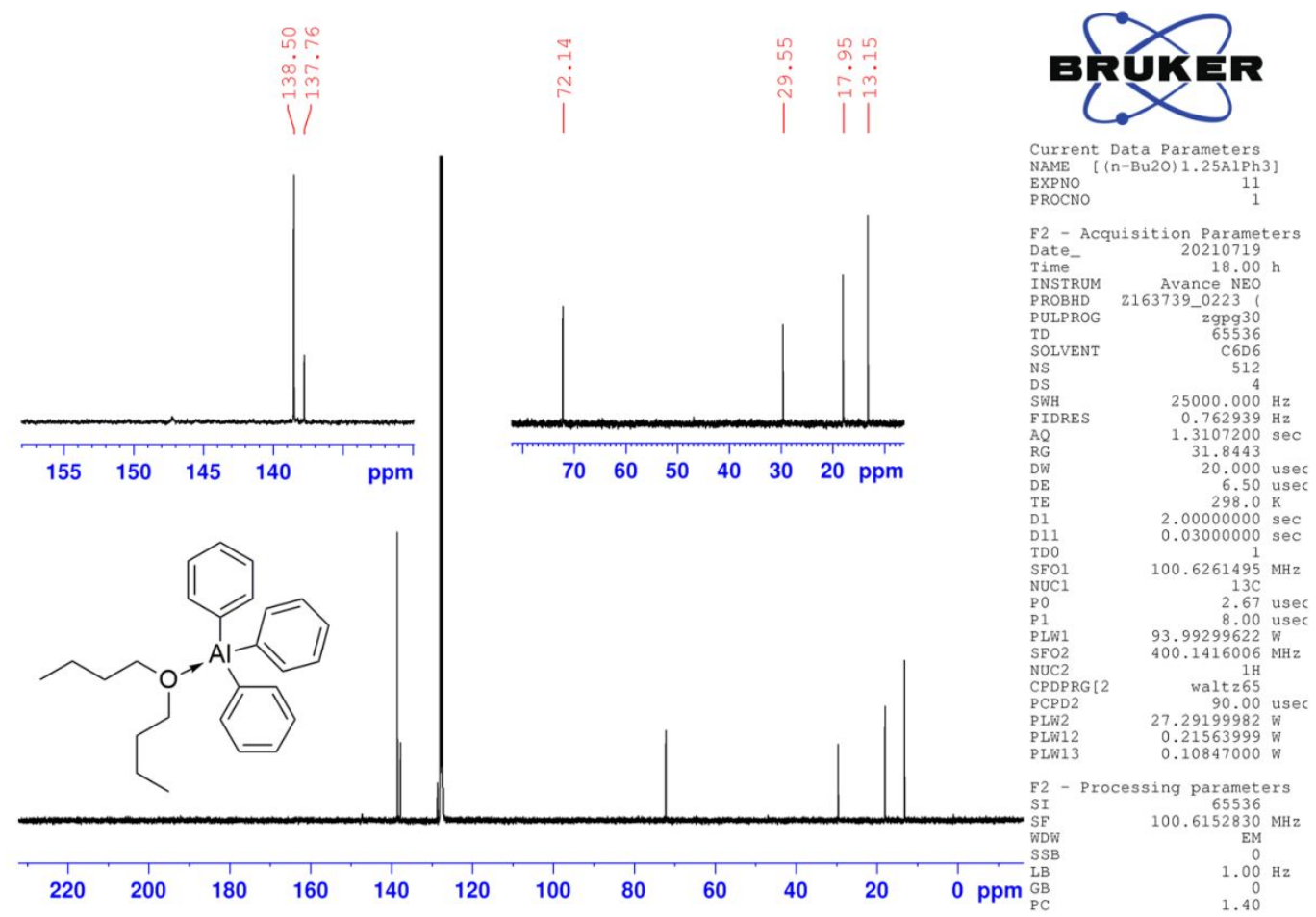

Figure S4. ${ }^{13} \mathrm{C}$ NMR spectrum of $\left[\left(n-\mathrm{Bu}_{2} \mathrm{O}\right)_{1.25} \mathrm{AlPh}_{3}\right]$ in $\mathrm{C}_{6} \mathrm{D}_{6}$ solution. 
S1.3 $[\mathrm{IPrH}]\left[\mathrm{Me}_{3} \mathrm{AlF}\right](1)$

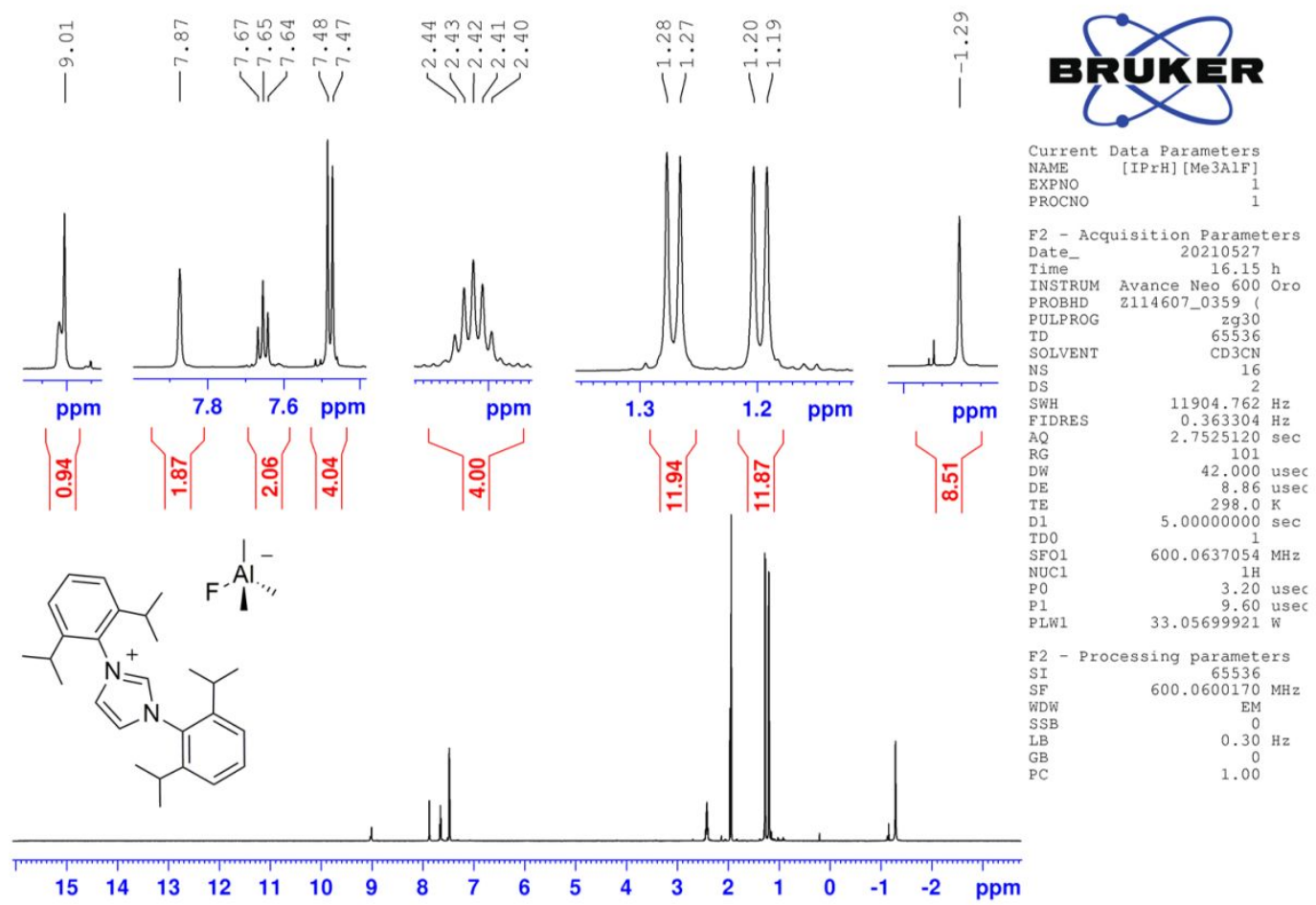

Figure S5. ${ }^{1} \mathrm{H}$ NMR spectrum of $[\mathrm{IPrH}]\left[\mathrm{Me}_{3} \mathrm{AlF}\right](\mathbf{1})$ in acetonitrile solution.

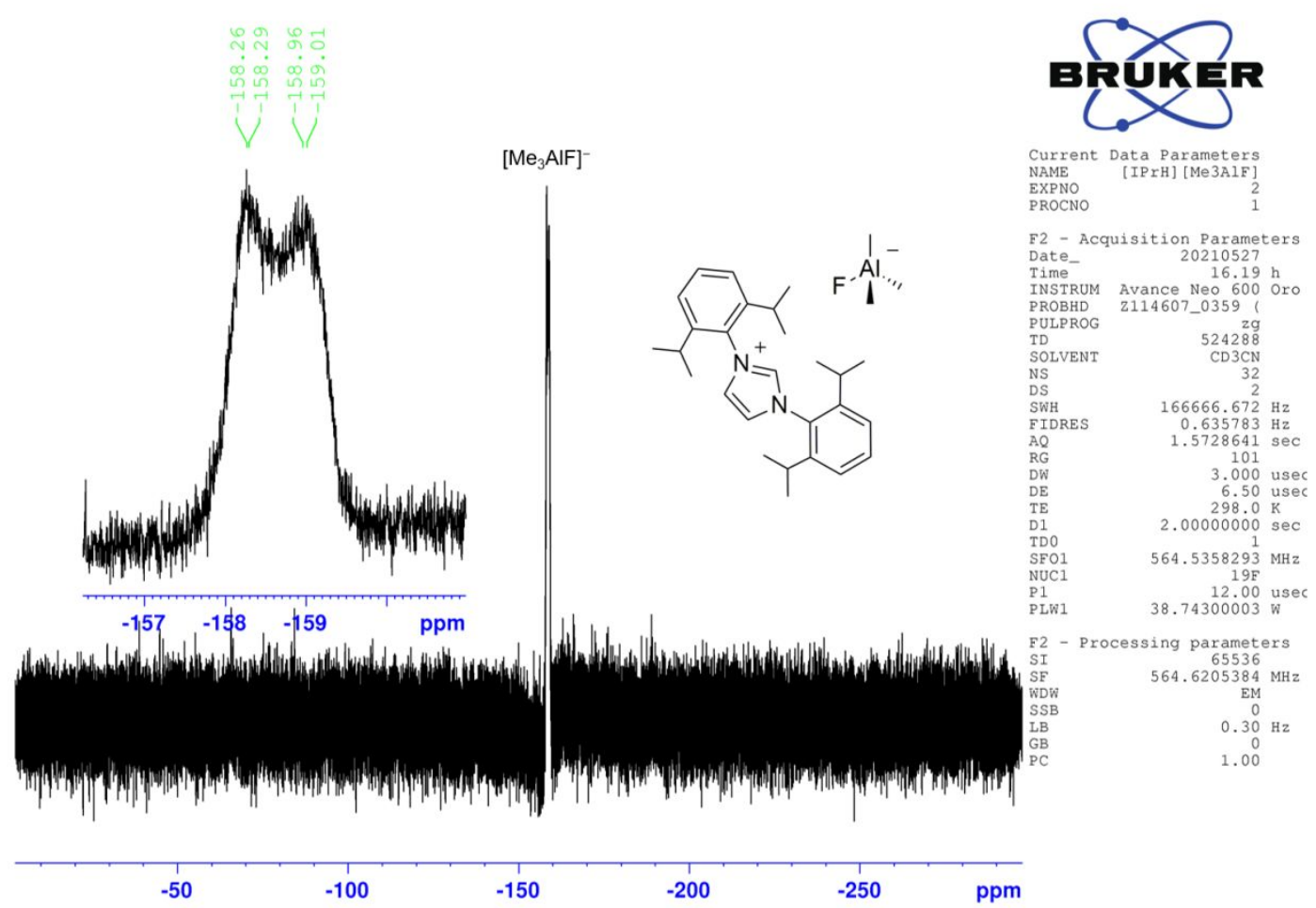

Figure S6. ${ }^{19} \mathrm{~F}$ NMR spectrum of $[\mathrm{IPrH}]\left[\mathrm{Me}_{3} \mathrm{AlF}\right](\mathbf{1})$ in acetonitrile solution. 


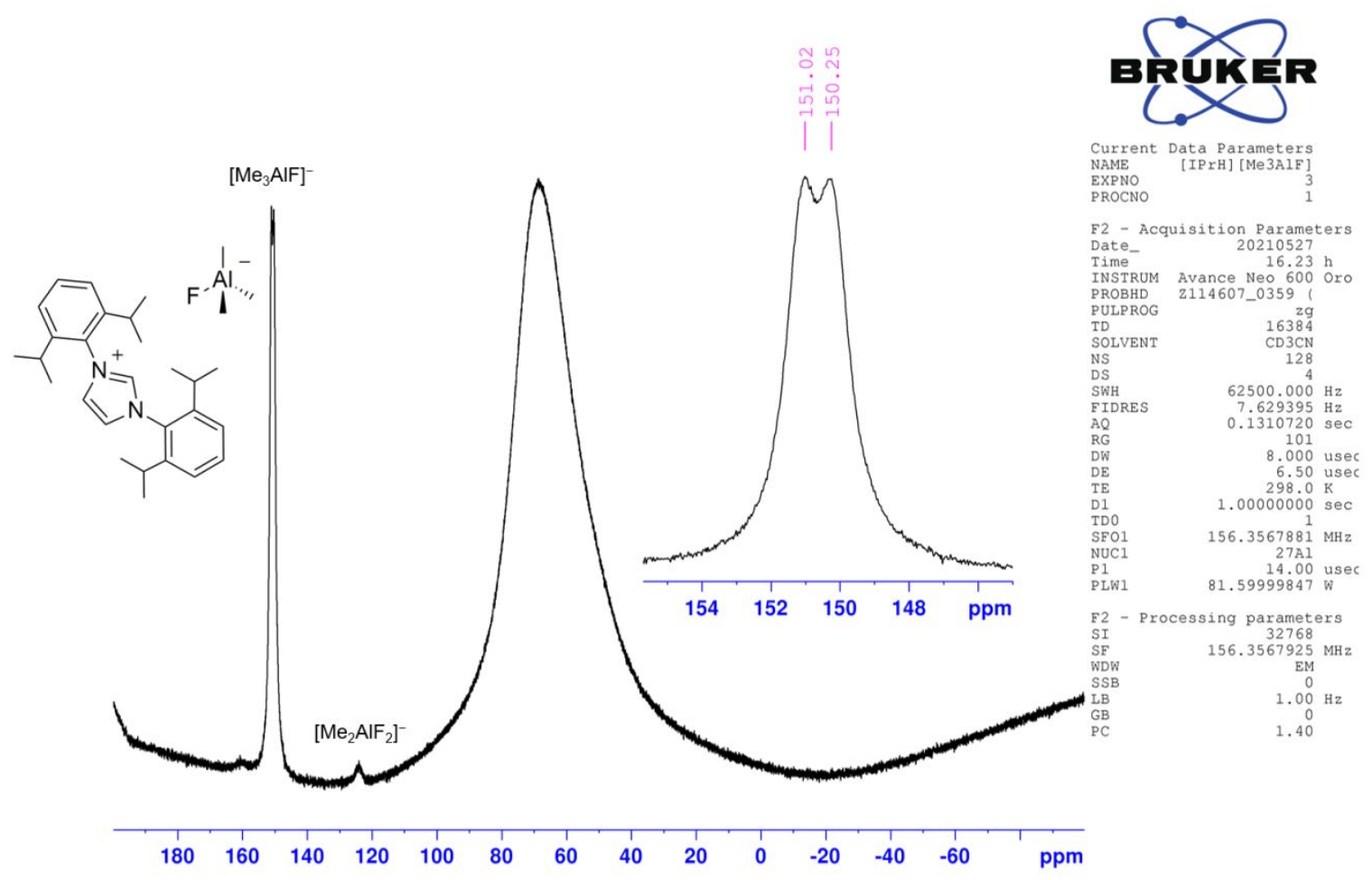

Figure S7. ${ }^{27} \mathrm{Al} \mathrm{NMR}$ spectrum of $[\mathrm{IPrH}]\left[\mathrm{Me}_{3} \mathrm{AlF}\right](\mathbf{1})$ in acetonitrile solution.

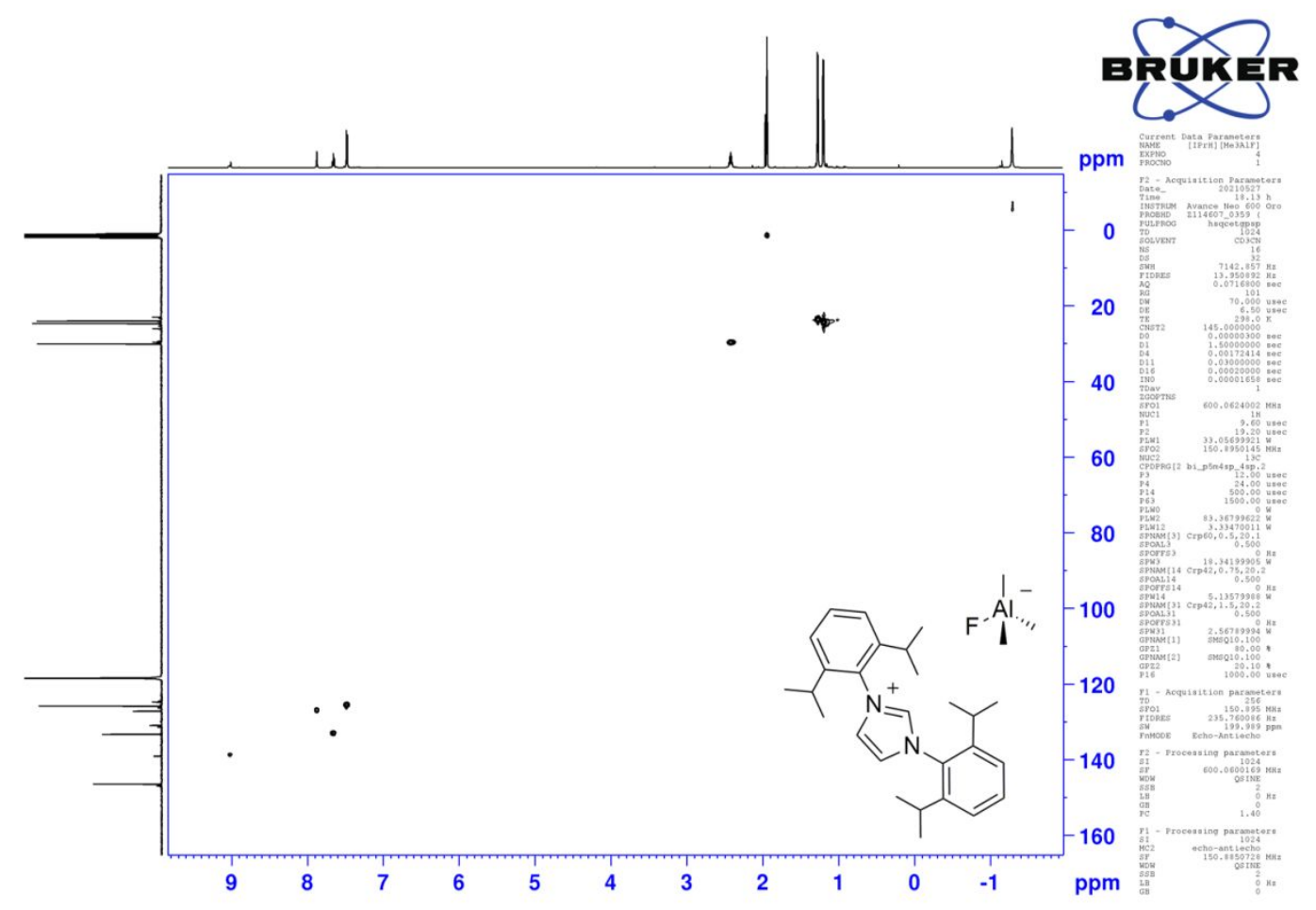

Figure S8. ${ }^{1} \mathrm{H}^{-13} \mathrm{C}$ HSQC NMR spectrum of $[\mathrm{IPrH}]\left[\mathrm{Me}_{3} \mathrm{AlF}\right](\mathbf{1})$ in acetonitrile solution. 


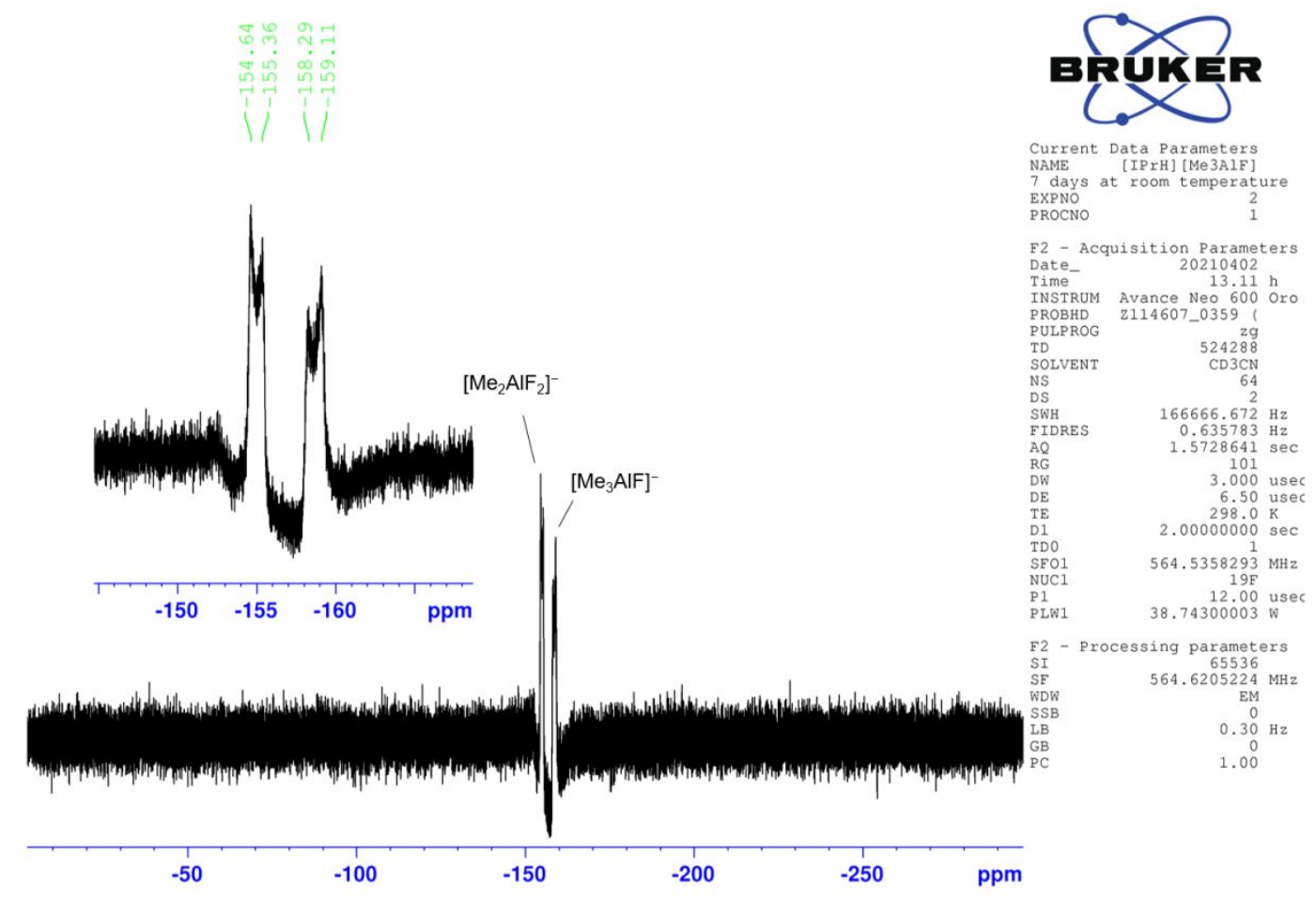

Figure S9. ${ }^{19} \mathrm{~F}$ NMR spectrum of $[\mathrm{IPrH}]\left[\mathrm{Me}_{3} \mathrm{AlF}\right]$ (1) stored for 7 days in acetonitrile solution at room temperature inside a glovebox.

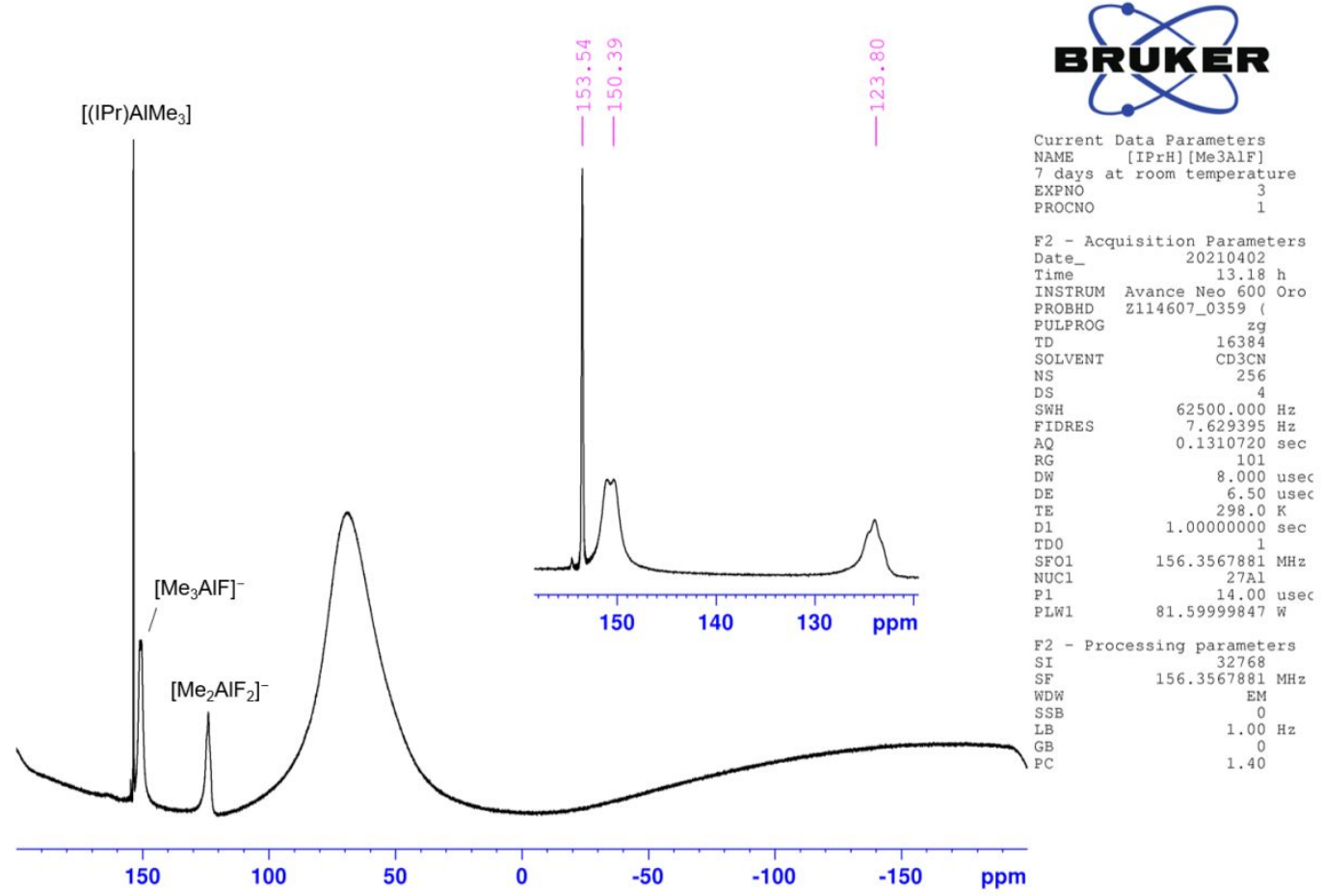

Figure S10. ${ }^{27} \mathrm{Al}$ NMR spectrum of [IPrH] $\left[\mathrm{Me}_{3} \mathrm{AlF}\right]$ (1) stored for 7 days in acetonitrile solution at room temperature inside a glovebox. 
$\mathrm{S1.5}[\mathrm{IPrH}]\left[\mathrm{Me}_{2} \mathrm{AlF}_{2}\right](2)$

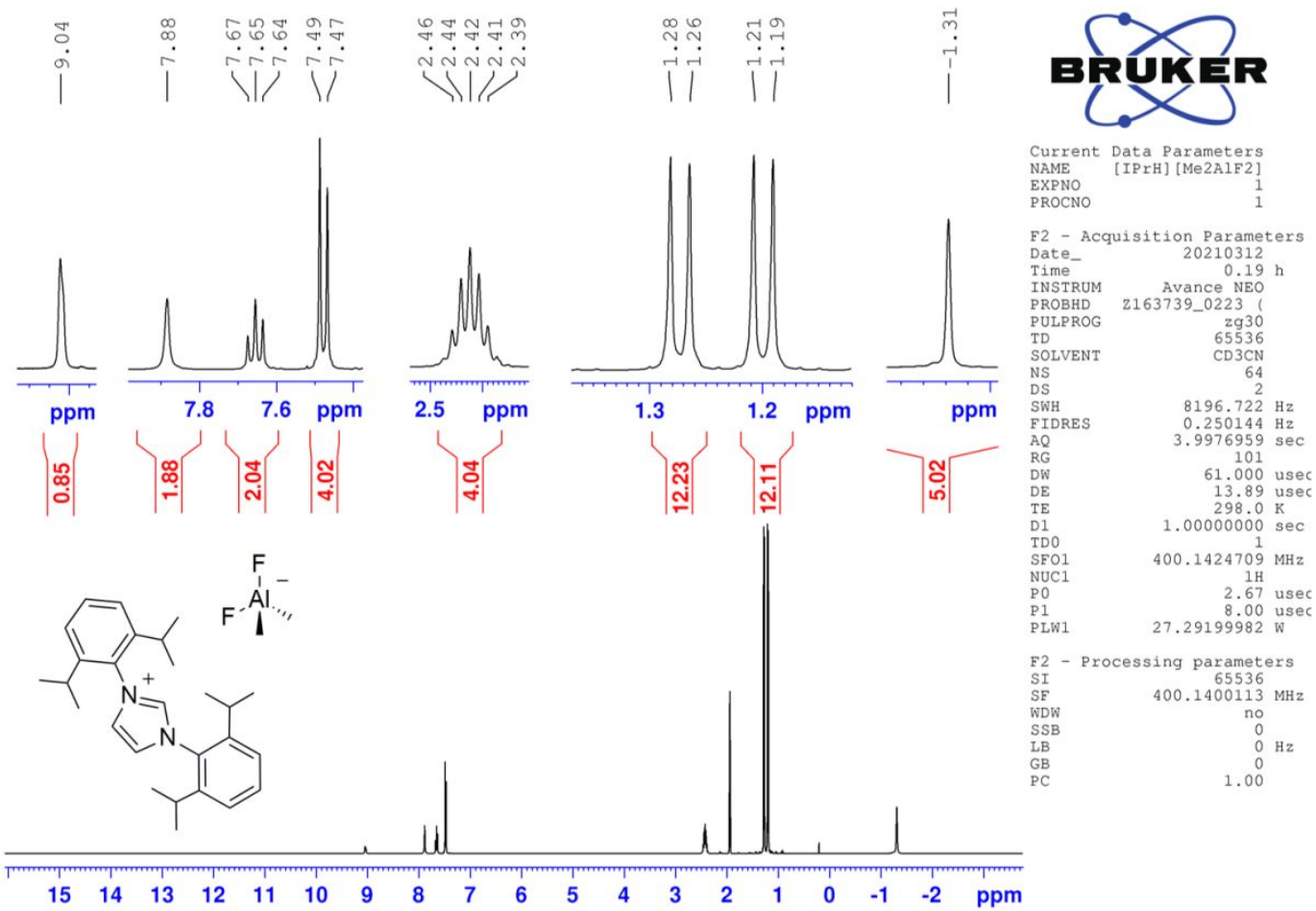

Figure S11. ${ }^{1} \mathrm{H}$ NMR spectrum of $[\mathrm{IPrH}]\left[\mathrm{Me}_{2} \mathrm{AlF}_{2}\right](2)$ in acetonitrile solution.

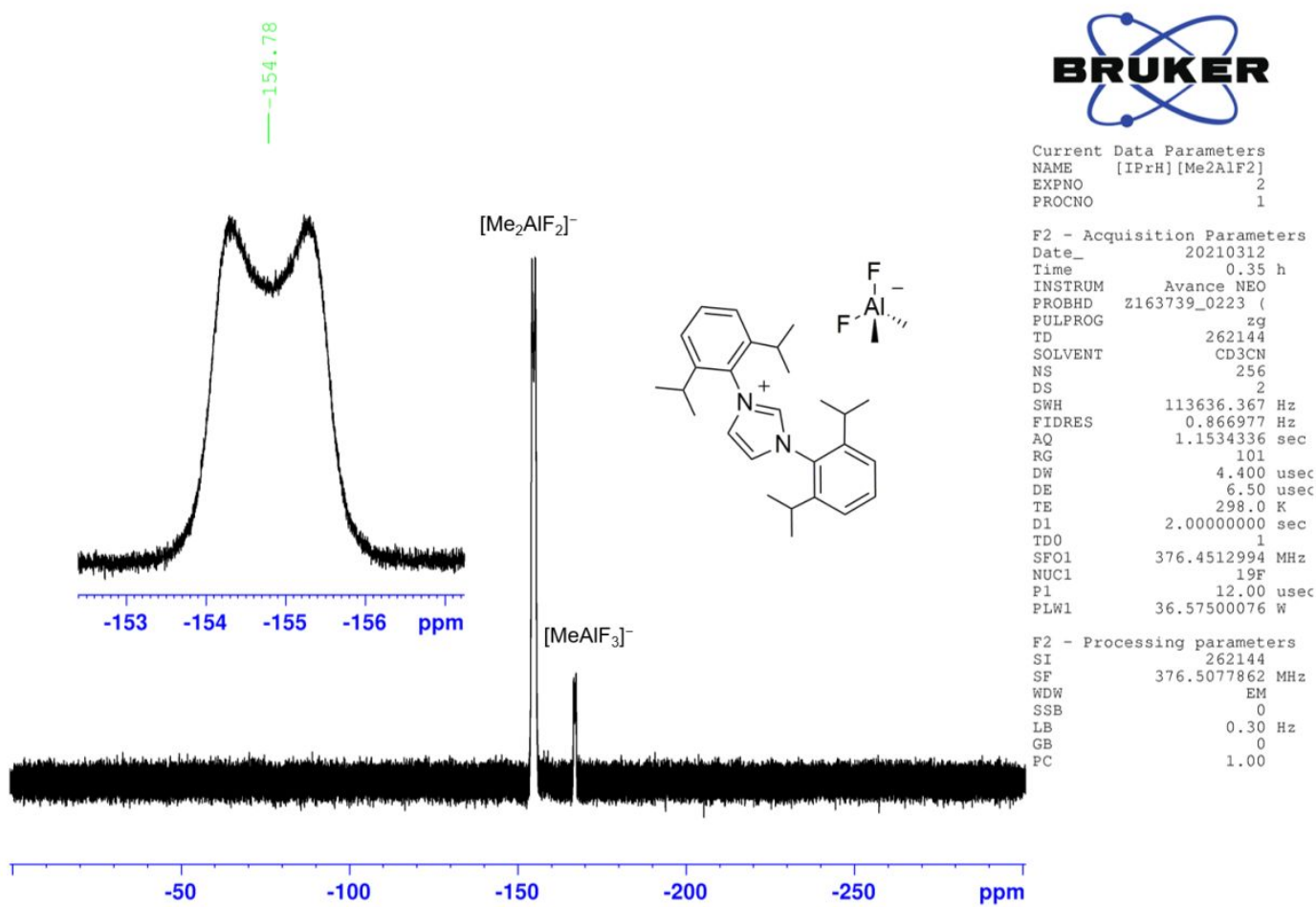

Figure S12. ${ }^{19} \mathrm{~F}$ NMR spectrum of $[\mathrm{IPrH}]\left[\mathrm{Me}_{2} \mathrm{AlF}_{2}\right](\mathbf{2})$ in acetonitrile solution. 


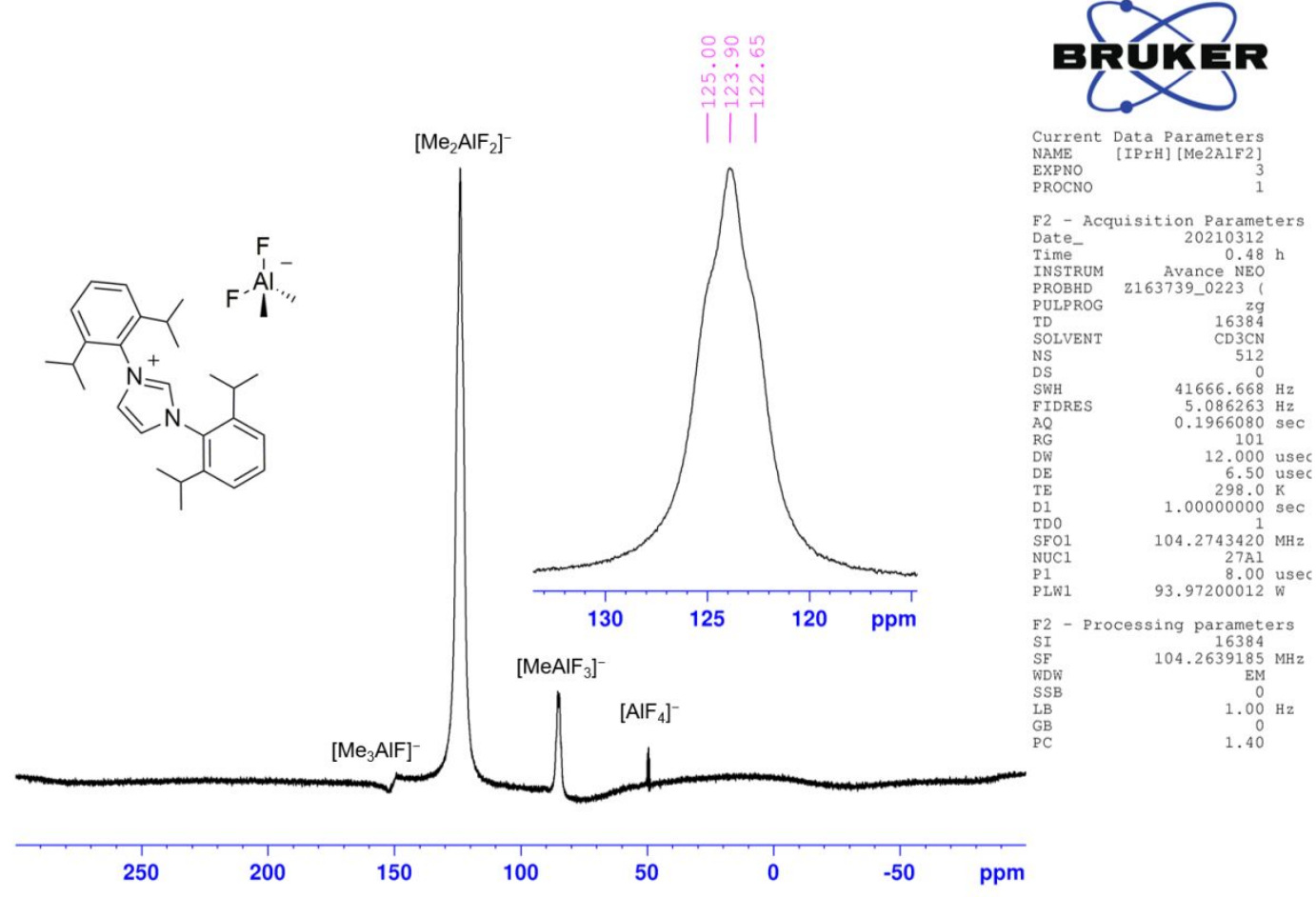

Figure S13. ${ }^{27} \mathrm{Al}$ NMR spectrum of $[\mathrm{IPrH}]\left[\mathrm{Me}_{2} \mathrm{AlF}_{2}\right](\mathbf{2})$ in acetonitrile solution.

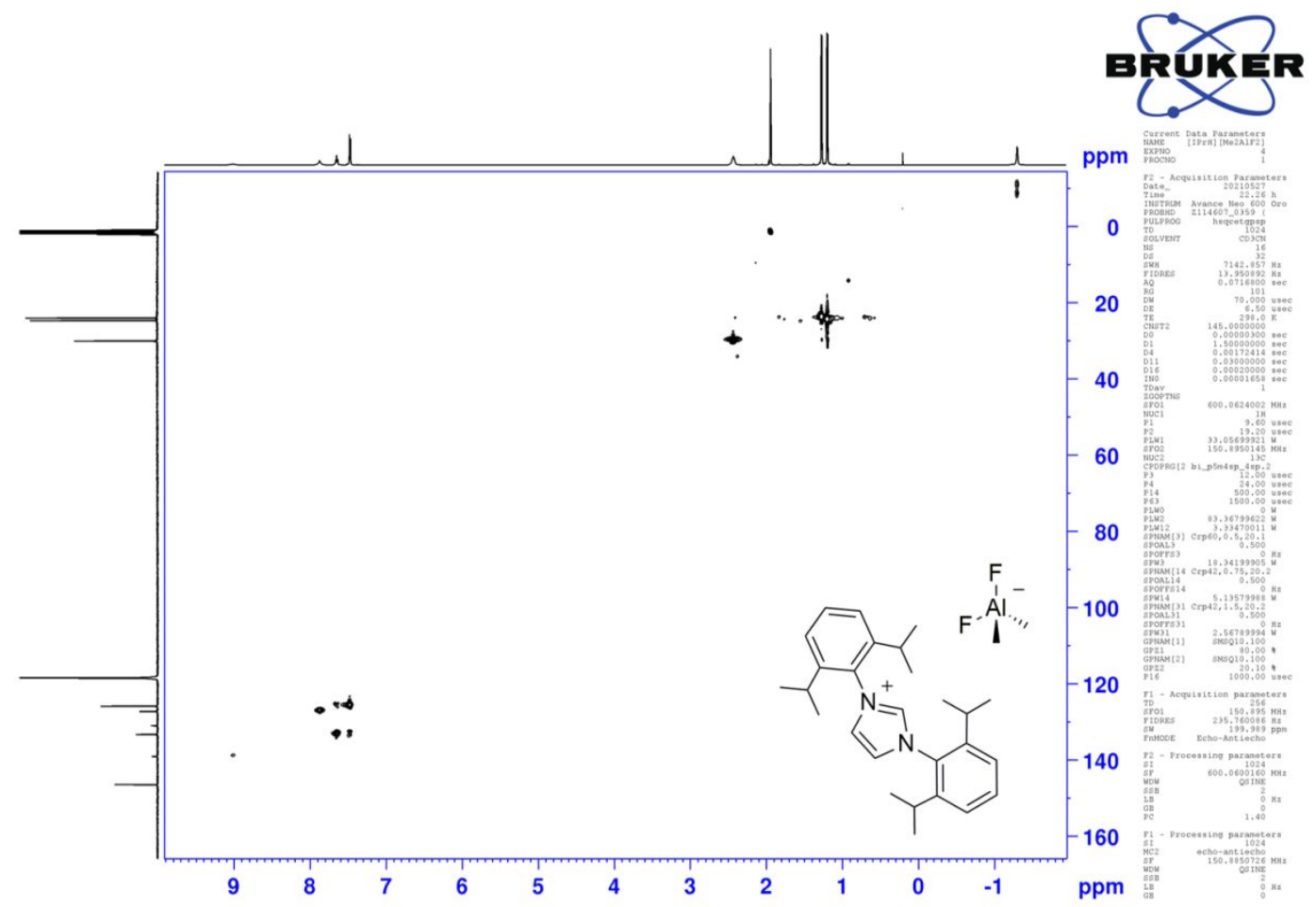

Figure S14. ${ }^{1} \mathrm{H}^{-13} \mathrm{C}$ HSQC NMR spectrum of $[\mathrm{IPrH}]\left[\mathrm{Me}_{2} \mathrm{AlF}_{2}\right](2)$ in acetonitrile solution. 
S1.6 [IPrH][MeAlF $]$ (3)

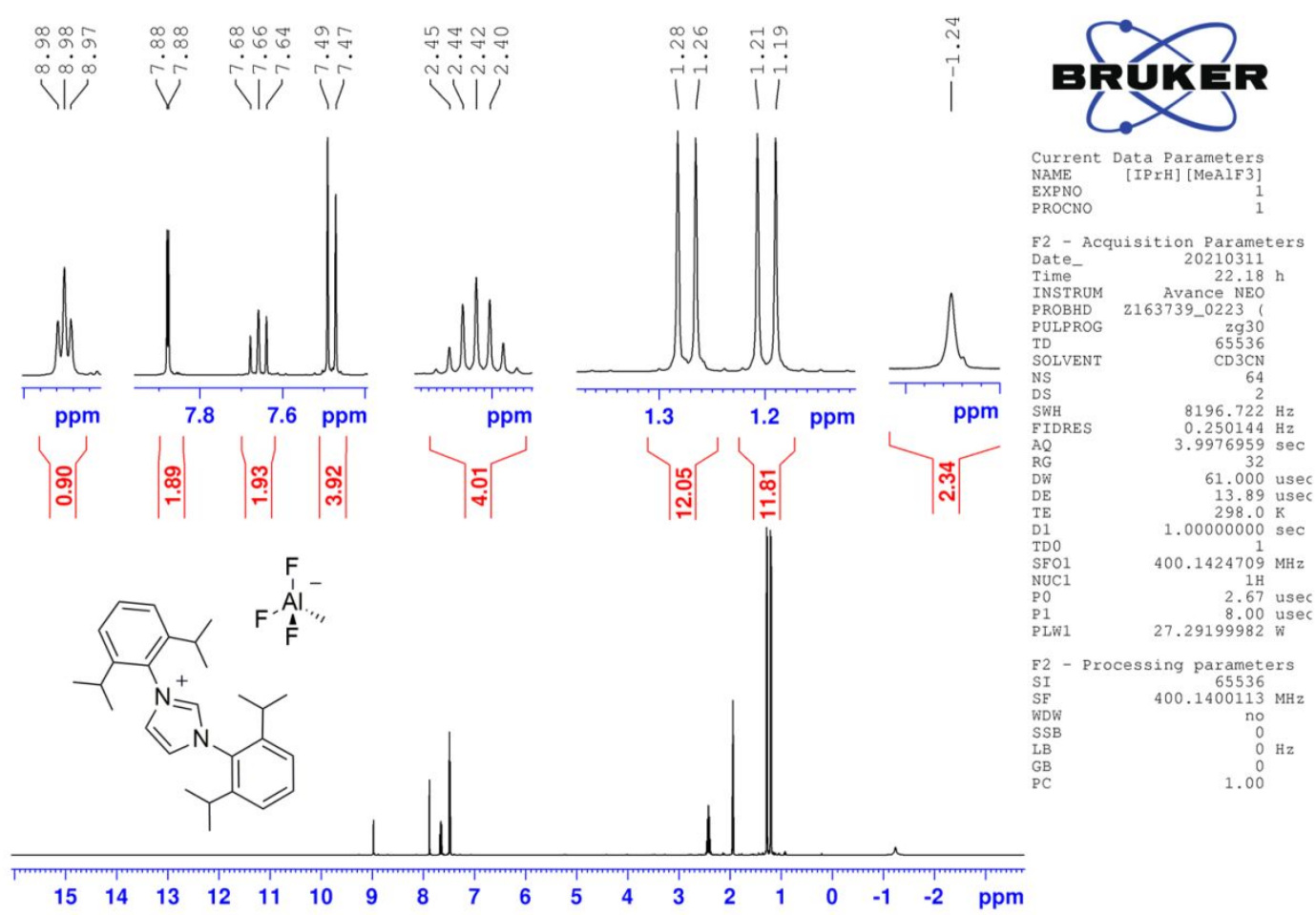

Figure S15. ${ }^{1} \mathrm{H}$ NMR spectrum of $[\mathrm{IPrH}]\left[\mathrm{MeAlF}_{3}\right](\mathbf{3})$ in acetonitrile solution.

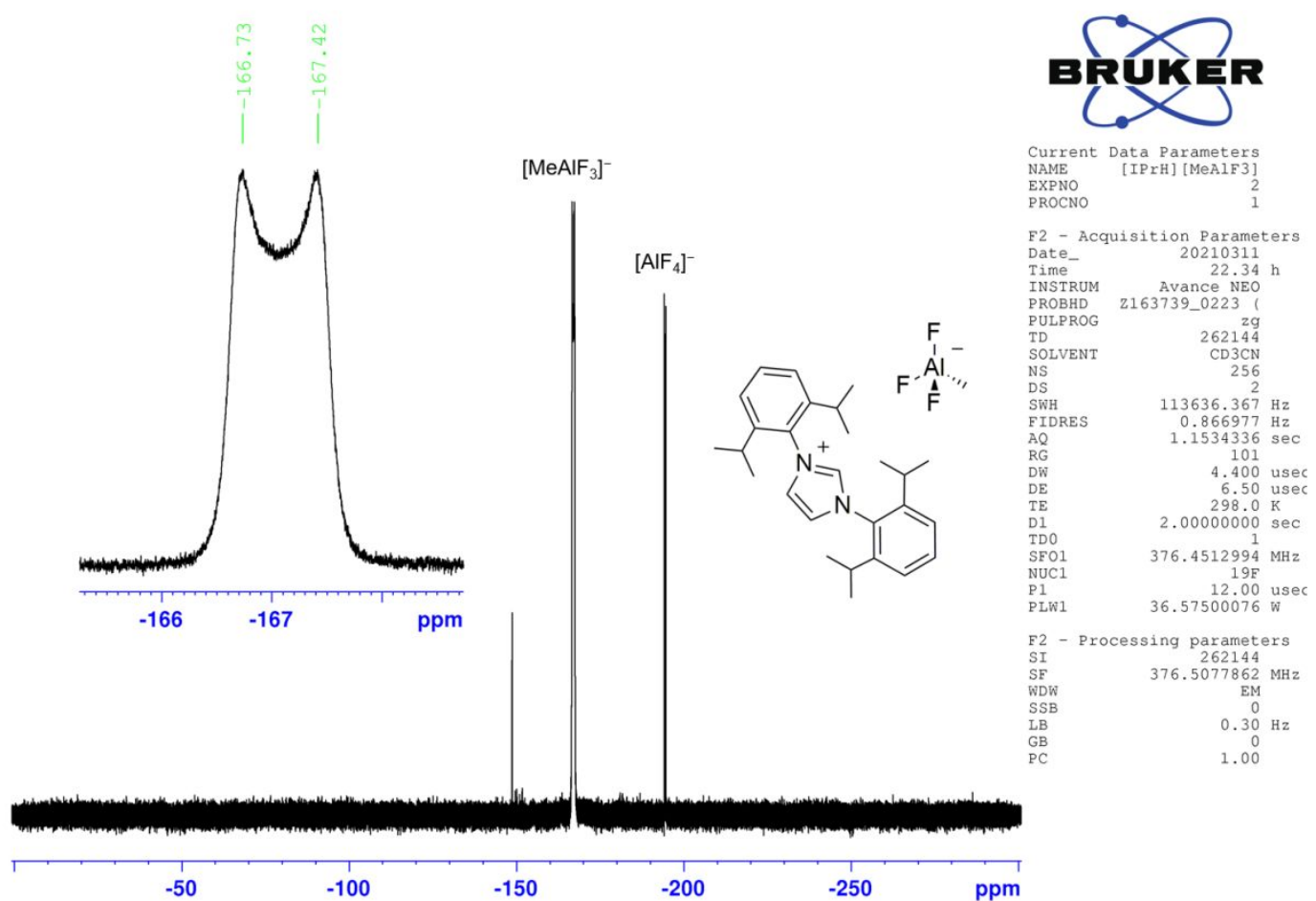

Figure S16. ${ }^{19} \mathrm{~F}$ NMR spectrum of $[\mathrm{IPrH}]\left[\mathrm{MeAlF}_{3}\right](3)$ in acetonitrile solution. 


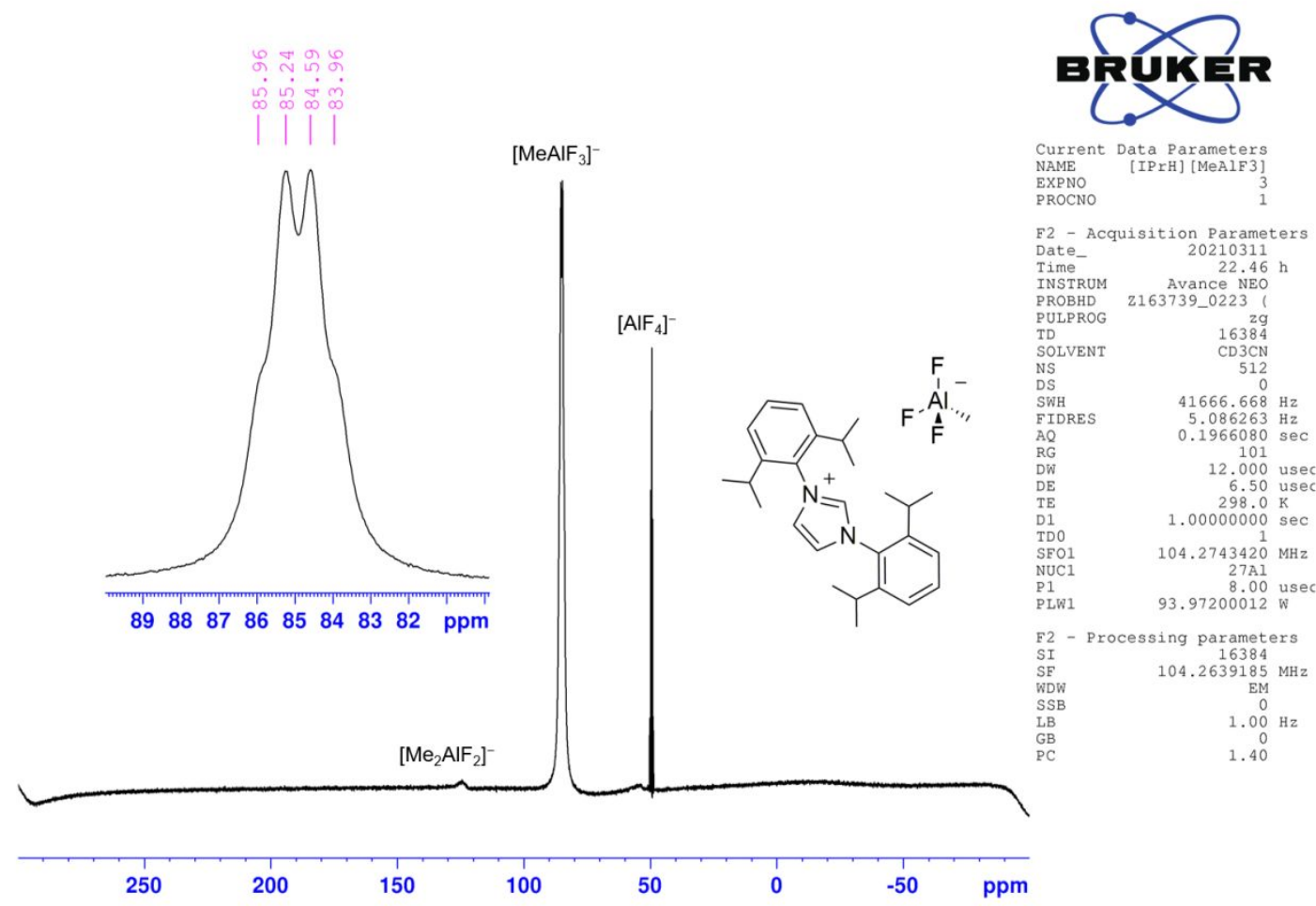

Figure S17. ${ }^{27} \mathrm{Al}$ NMR spectrum of $[\mathrm{IPrH}]\left[\mathrm{MeAlF}_{3}\right](\mathbf{3})$ in acetonitrile solution.

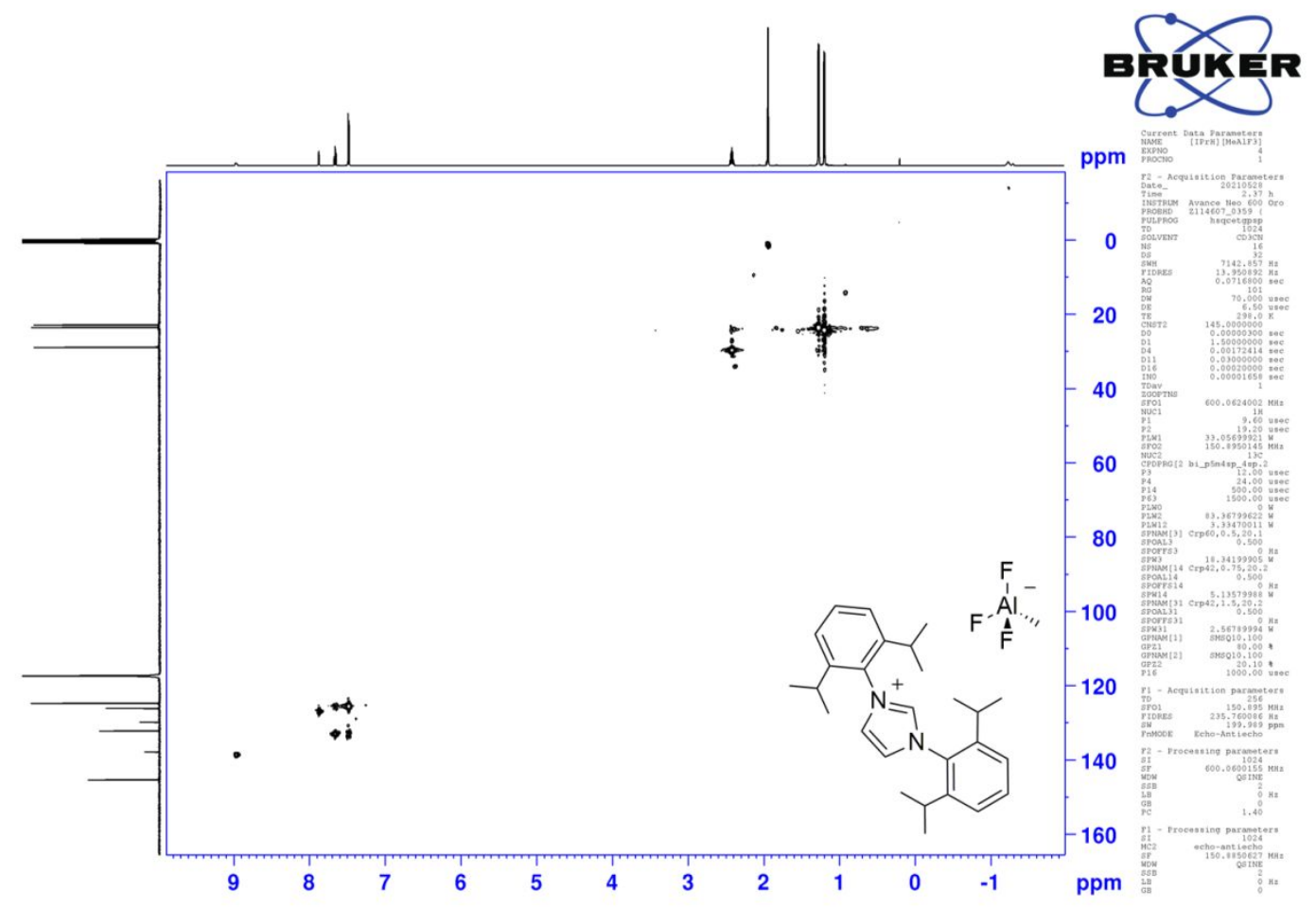

Figure S18. ${ }^{1} \mathrm{H}^{-13} \mathrm{C}$ HSQC NMR spectrum of $[\mathrm{IPrH}]\left[\mathrm{MeAlF}_{3}\right](3)$ in acetonitrile solution. 


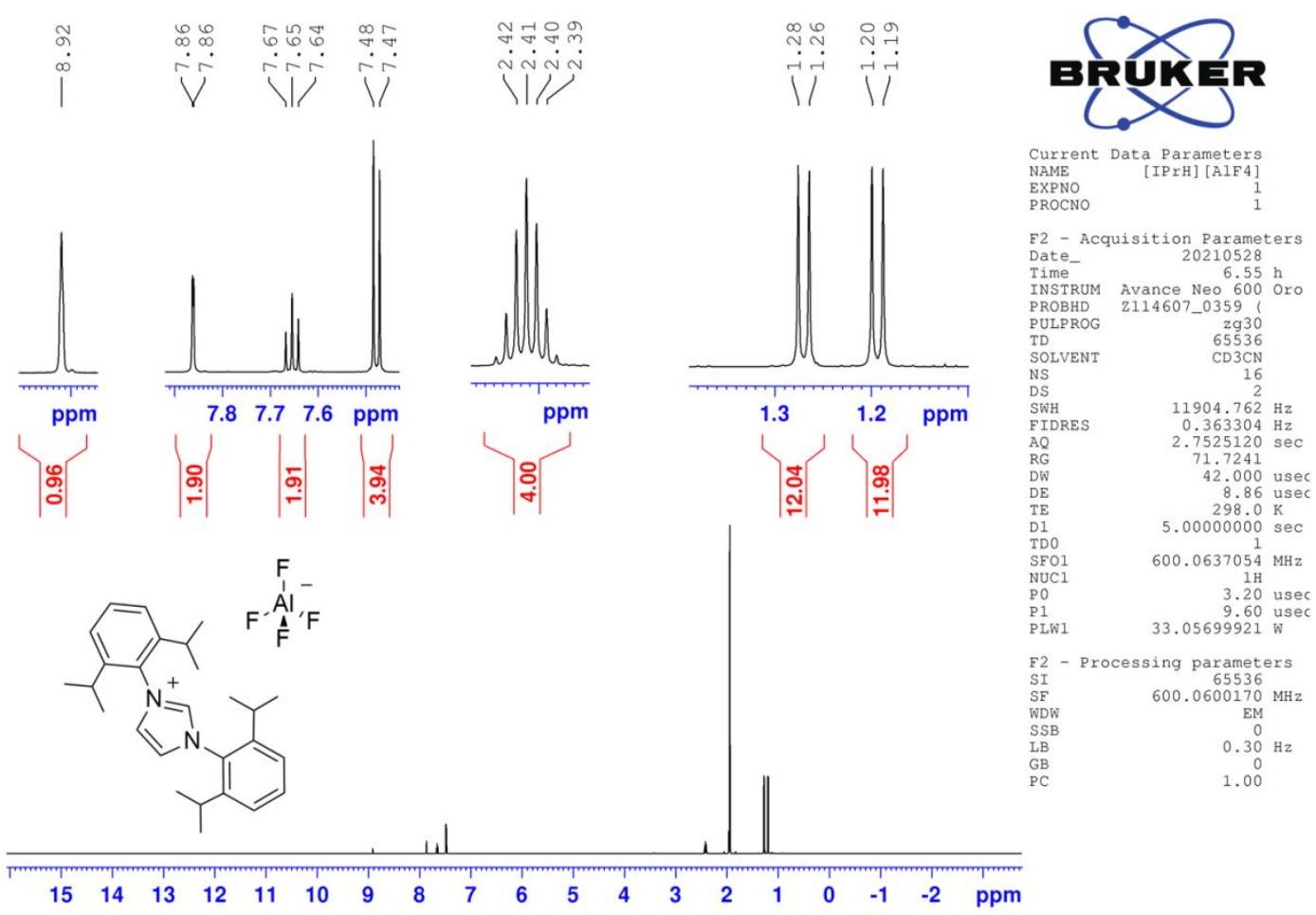

Figure S19. ${ }^{1} \mathrm{H}$ NMR spectrum of $[\mathrm{IPrH}]\left[\mathrm{AlF}_{4}\right](4)$ in acetonitrile solution.

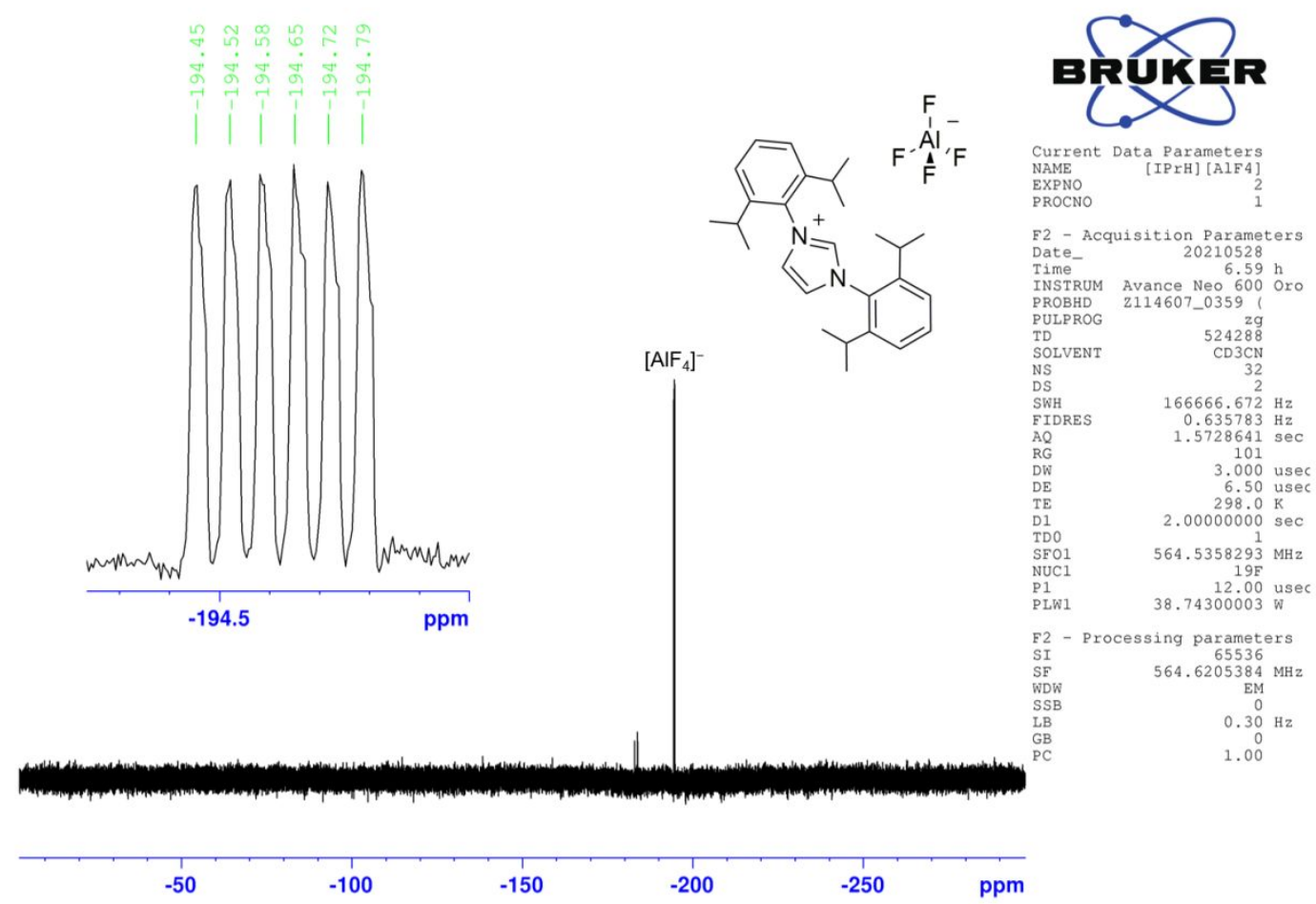

Figure S20. ${ }^{19} \mathrm{~F}$ NMR spectrum of $[\mathrm{IPrH}]\left[\mathrm{AlF}_{4}\right](4)$ in acetonitrile solution. 


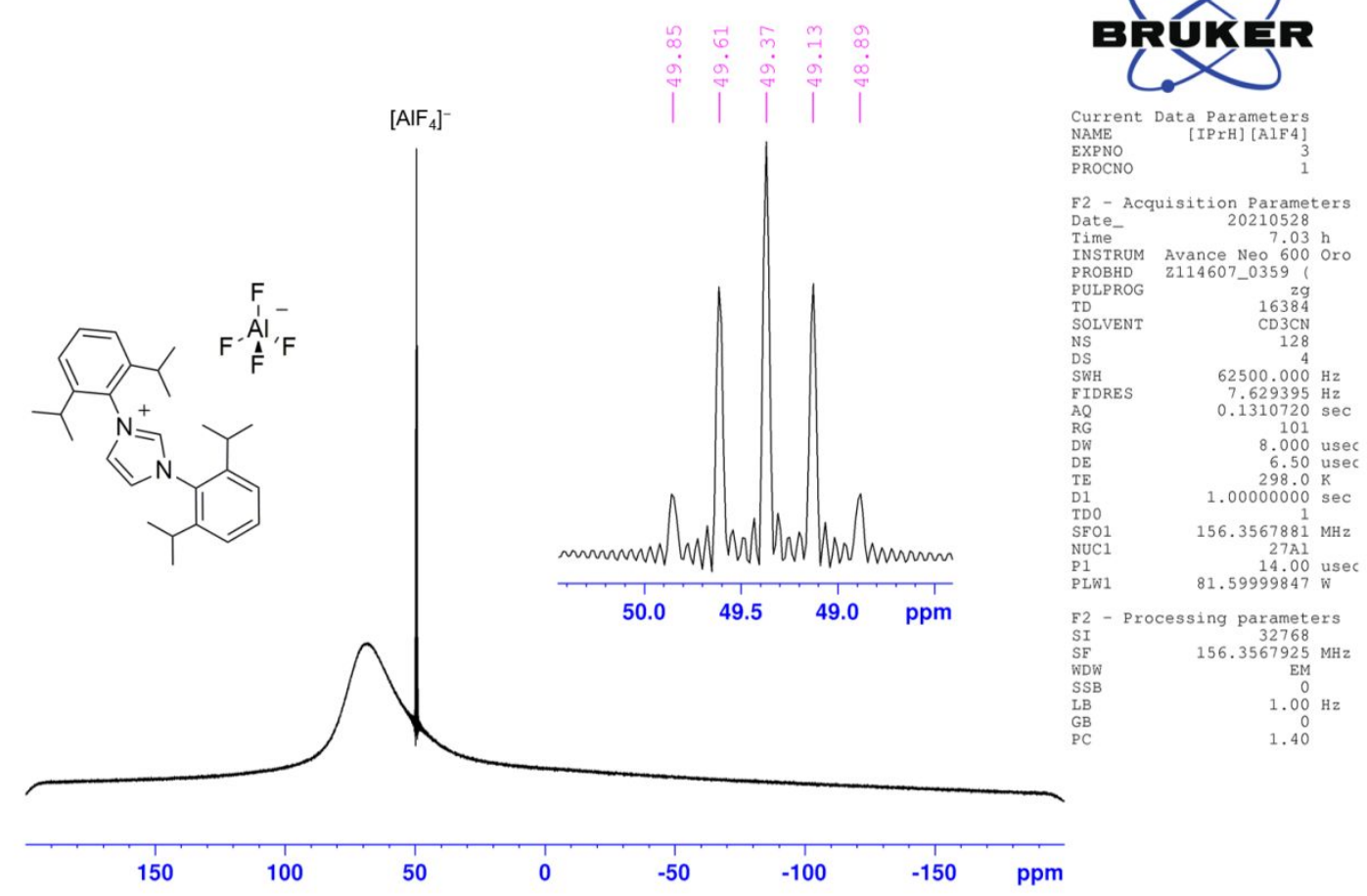

Figure S21. ${ }^{27} \mathrm{Al}$ NMR spectrum of $[\mathrm{IPrH}]\left[\mathrm{AlF}_{4}\right](\mathbf{4})$ in acetonitrile solution.

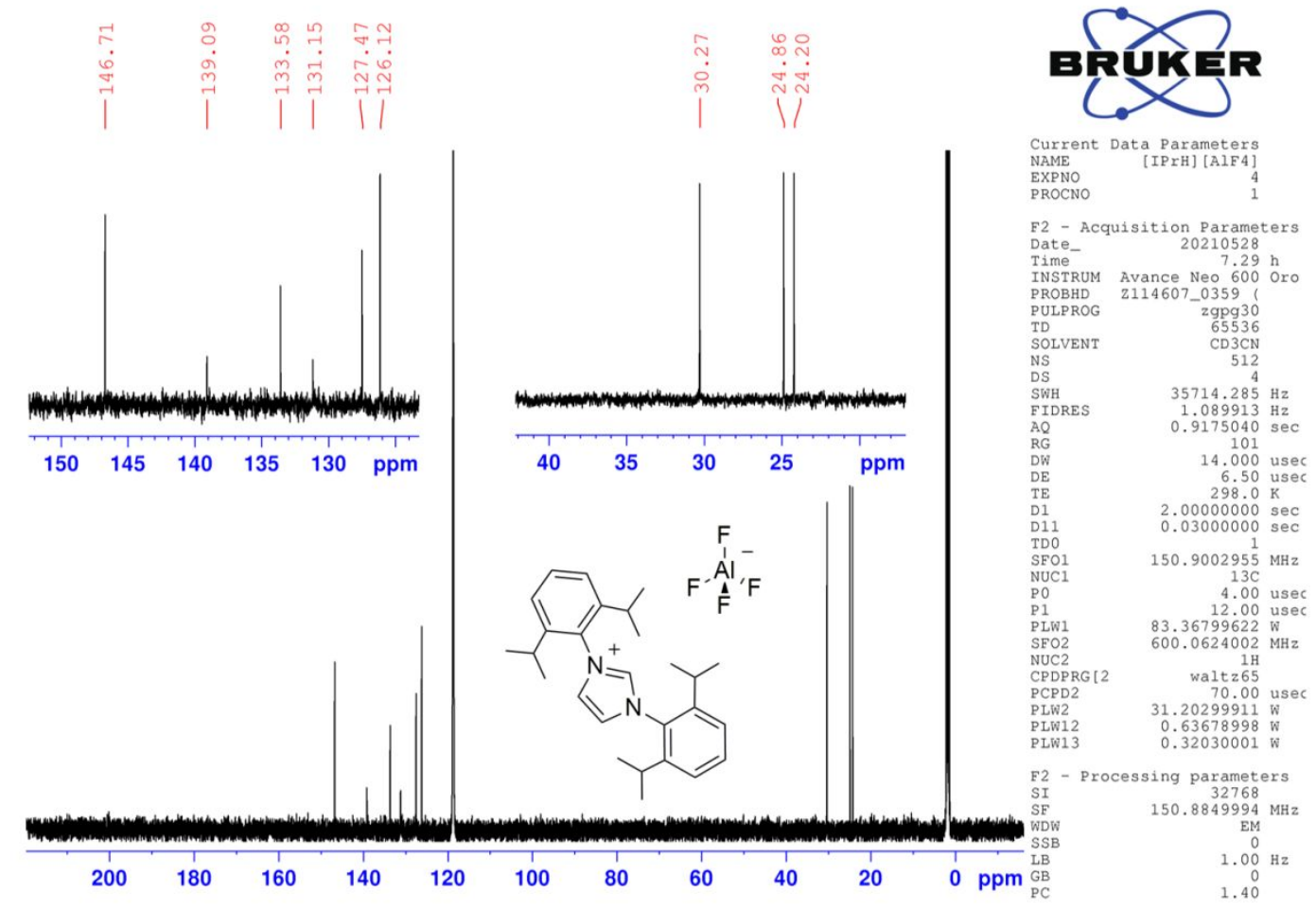

Figure S22. ${ }^{13} \mathrm{C}$ NMR spectrum of $[\mathrm{IPrH}]\left[\mathrm{AlF}_{4}\right](4)$ in acetonitrile solution. 
S1.8 $[I P r H]\left[\mathrm{n}-\mathrm{Bu}_{3} \mathrm{AlF}\right](5)$

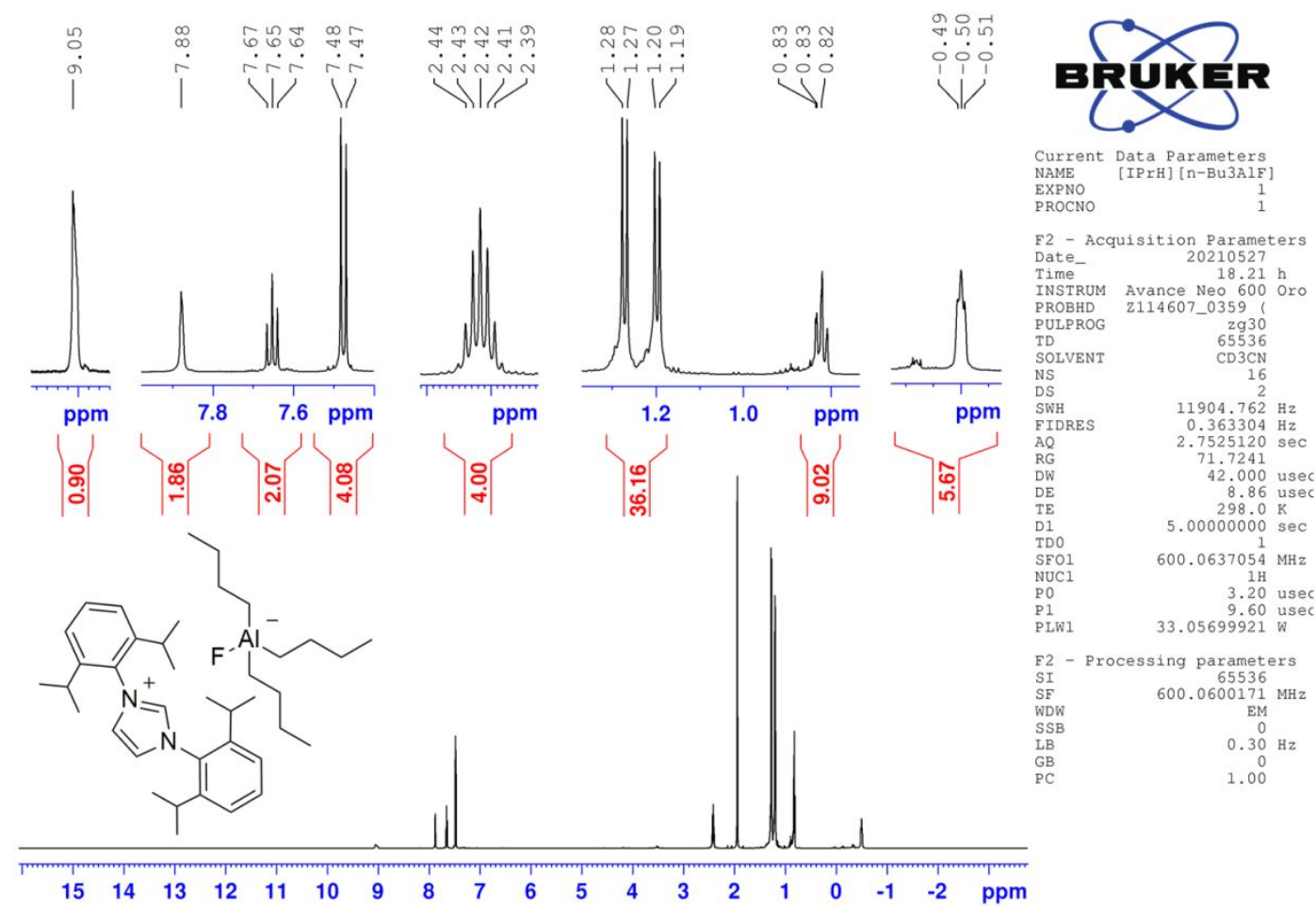

Figure S23. ${ }^{1} \mathrm{H}$ NMR spectrum of $[\mathrm{IPrH}]\left[n-\mathrm{Bu}_{3} \mathrm{AlF}\right](\mathbf{5})$ in acetonitrile solution.

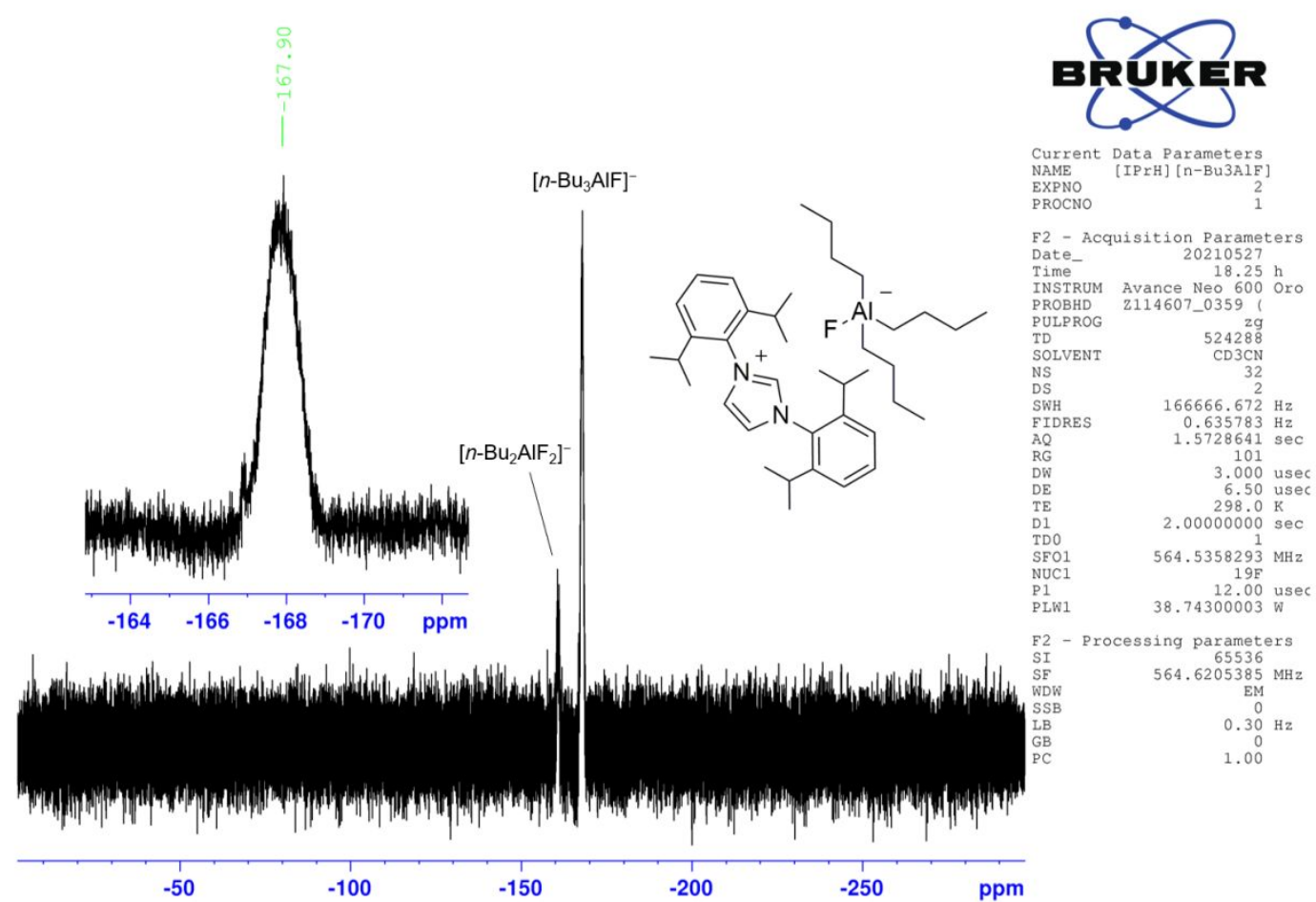

Figure S24. ${ }^{19} \mathrm{~F}$ NMR spectrum of $[\mathrm{IPrH}]\left[n-\mathrm{Bu}_{3} \mathrm{AlF}\right](\mathbf{5})$ in acetonitrile solution. 


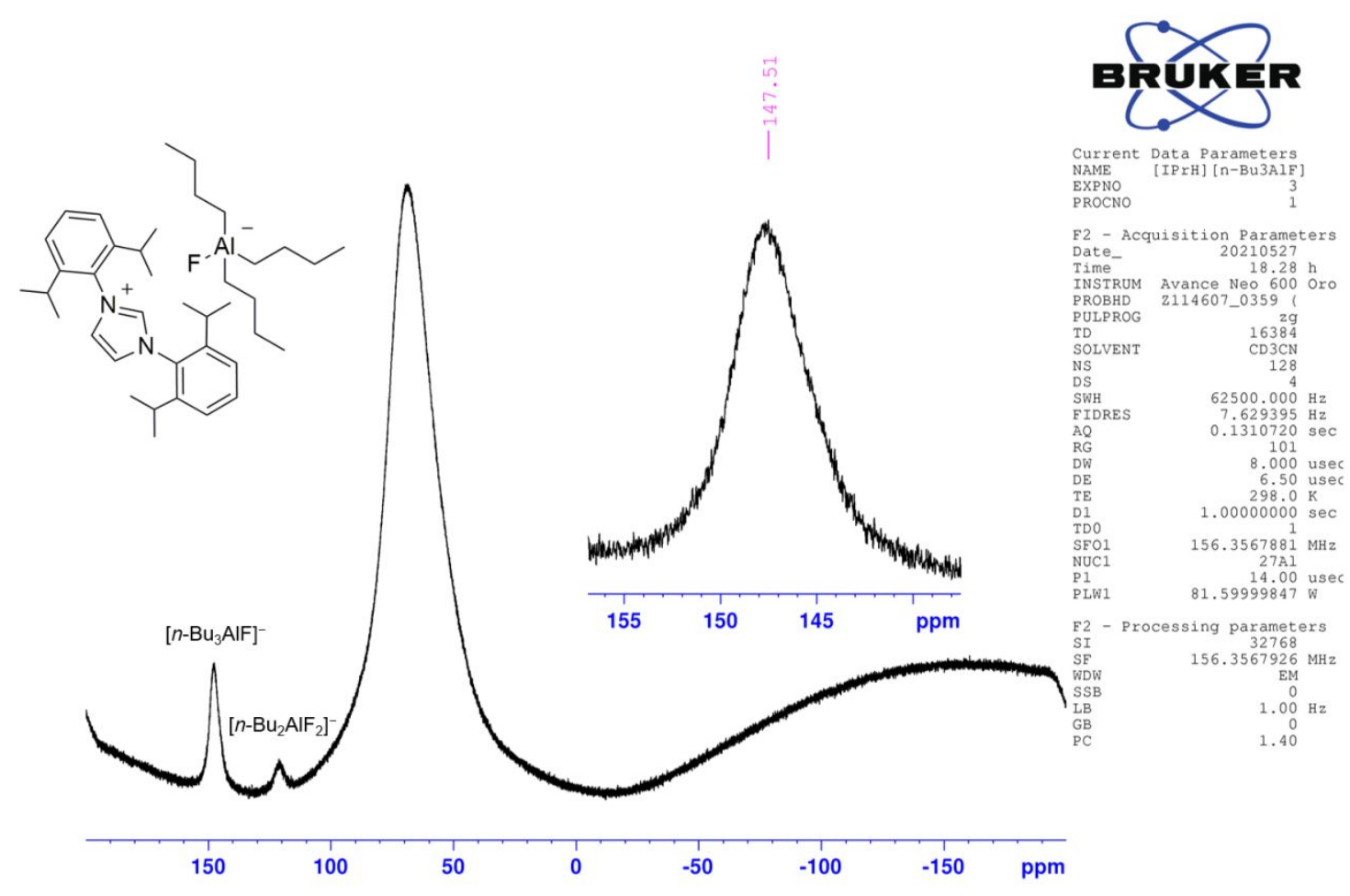

Figure S25. ${ }^{27} \mathrm{Al}$ NMR spectrum of $[\mathrm{IPrH}]\left[n-\mathrm{Bu}_{3} \mathrm{AlF}\right](\mathbf{5})$ in acetonitrile solution.

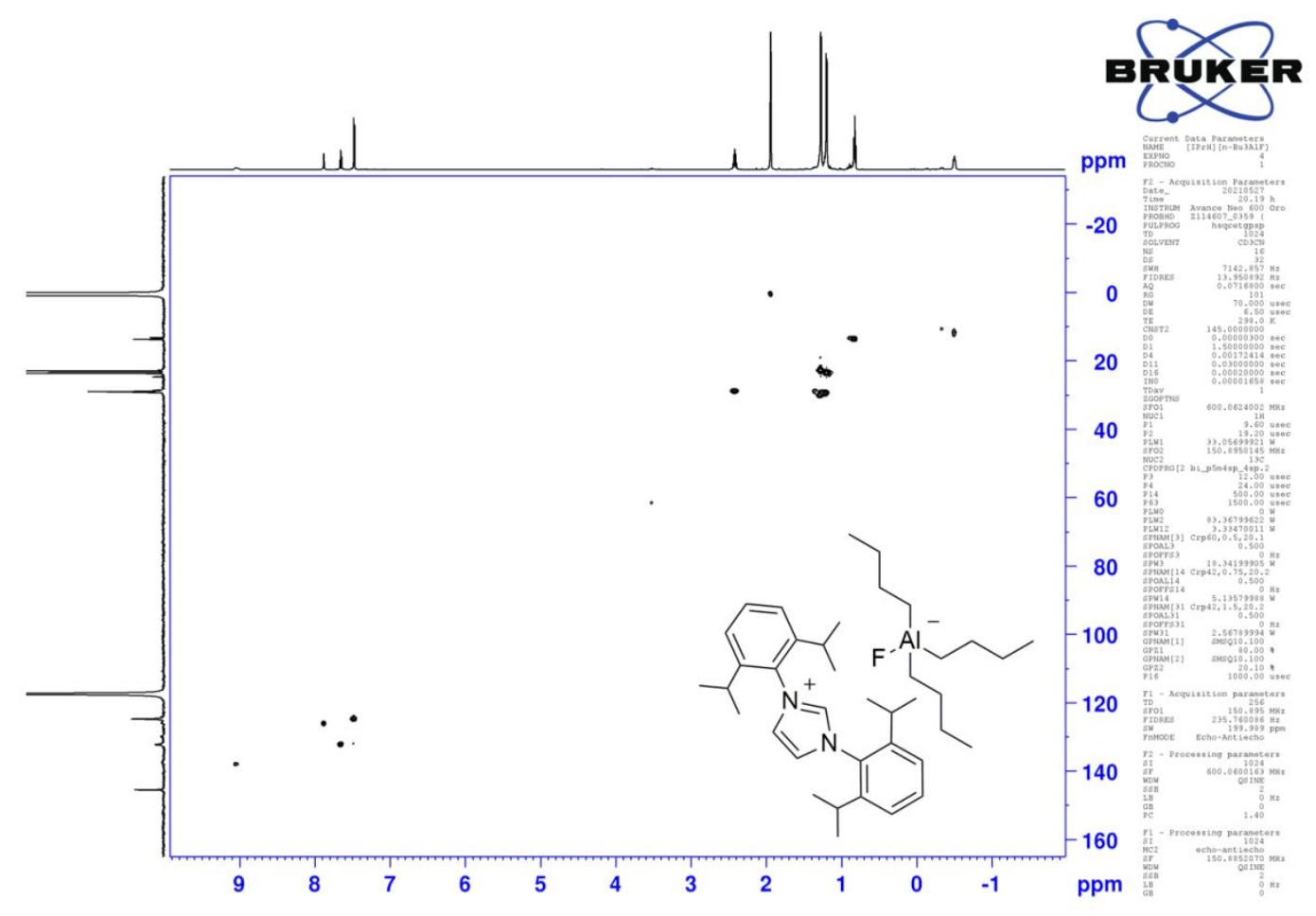

Figure S26. ${ }^{1} \mathrm{H}-{ }^{13} \mathrm{C}$ HSQC NMR spectrum of $[\mathrm{IPrH}]\left[n-\mathrm{Bu}_{3} \mathrm{AlF}\right](5)$ in acetonitrile solution. 


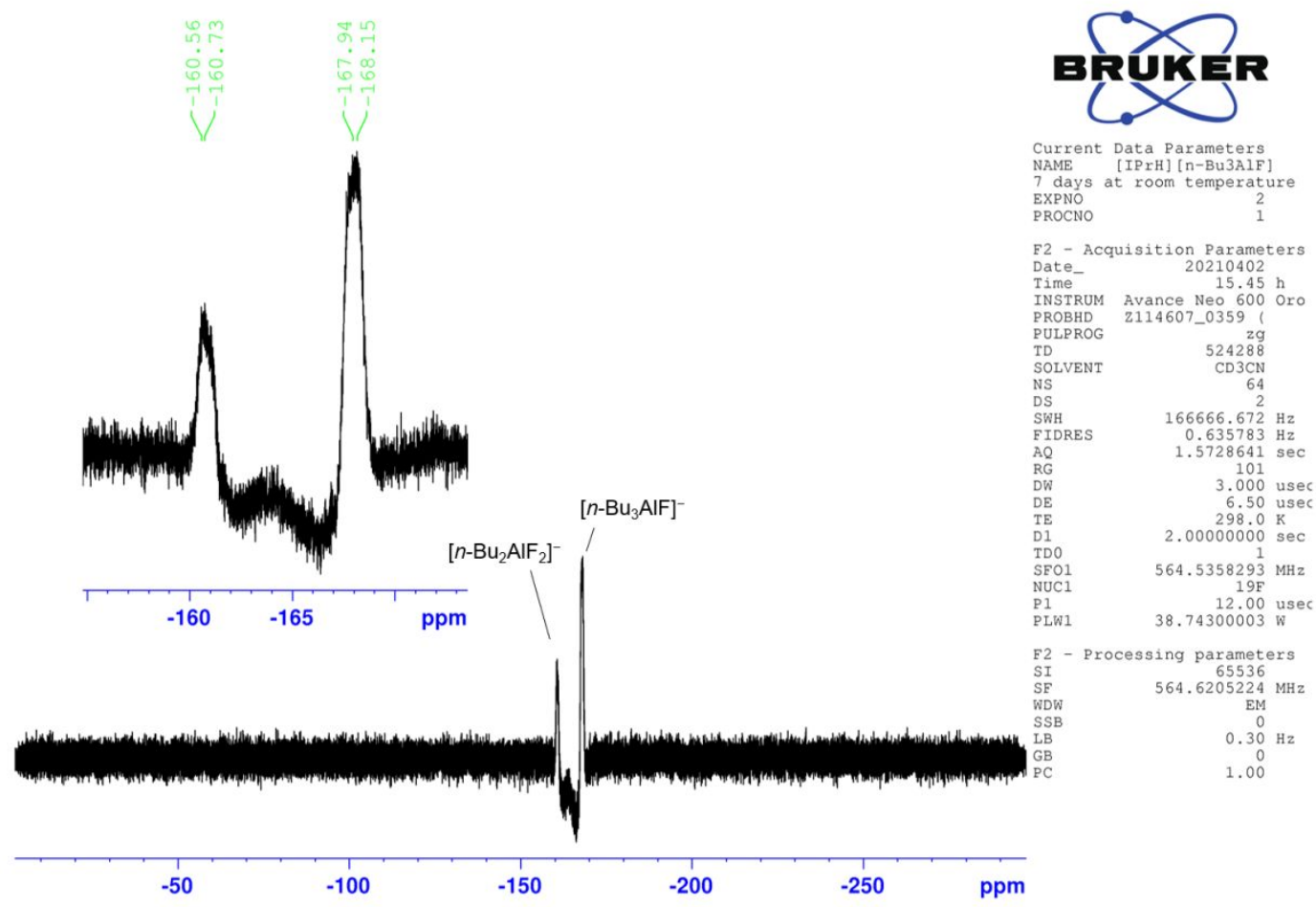

Figure S27. ${ }^{19} \mathrm{~F}$ NMR spectrum of $[\mathrm{IPrH}]\left[n-\mathrm{Bu}_{3} \mathrm{AlF}\right](5)$ stored for 7 days in acetonitrile solution at room temperature inside a glovebox.

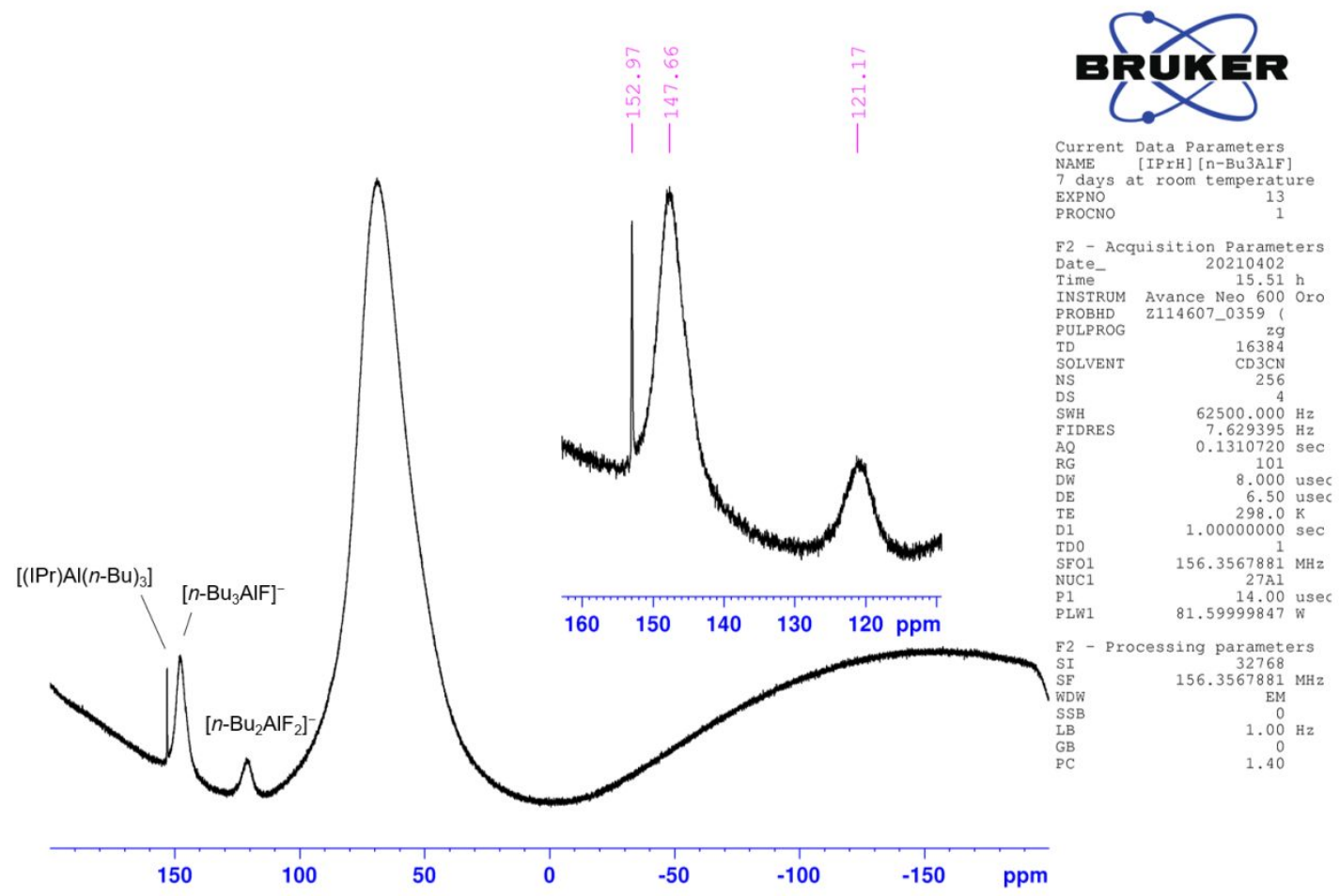

Figure S28. ${ }^{27} \mathrm{Al} \mathrm{NMR}$ spectrum of $[\mathrm{IPrH}]\left[n-\mathrm{Bu}_{3} \mathrm{AlF}\right](\mathbf{5})$ stored for 7 days in acetonitrile solution at room temperature inside a glovebox. 


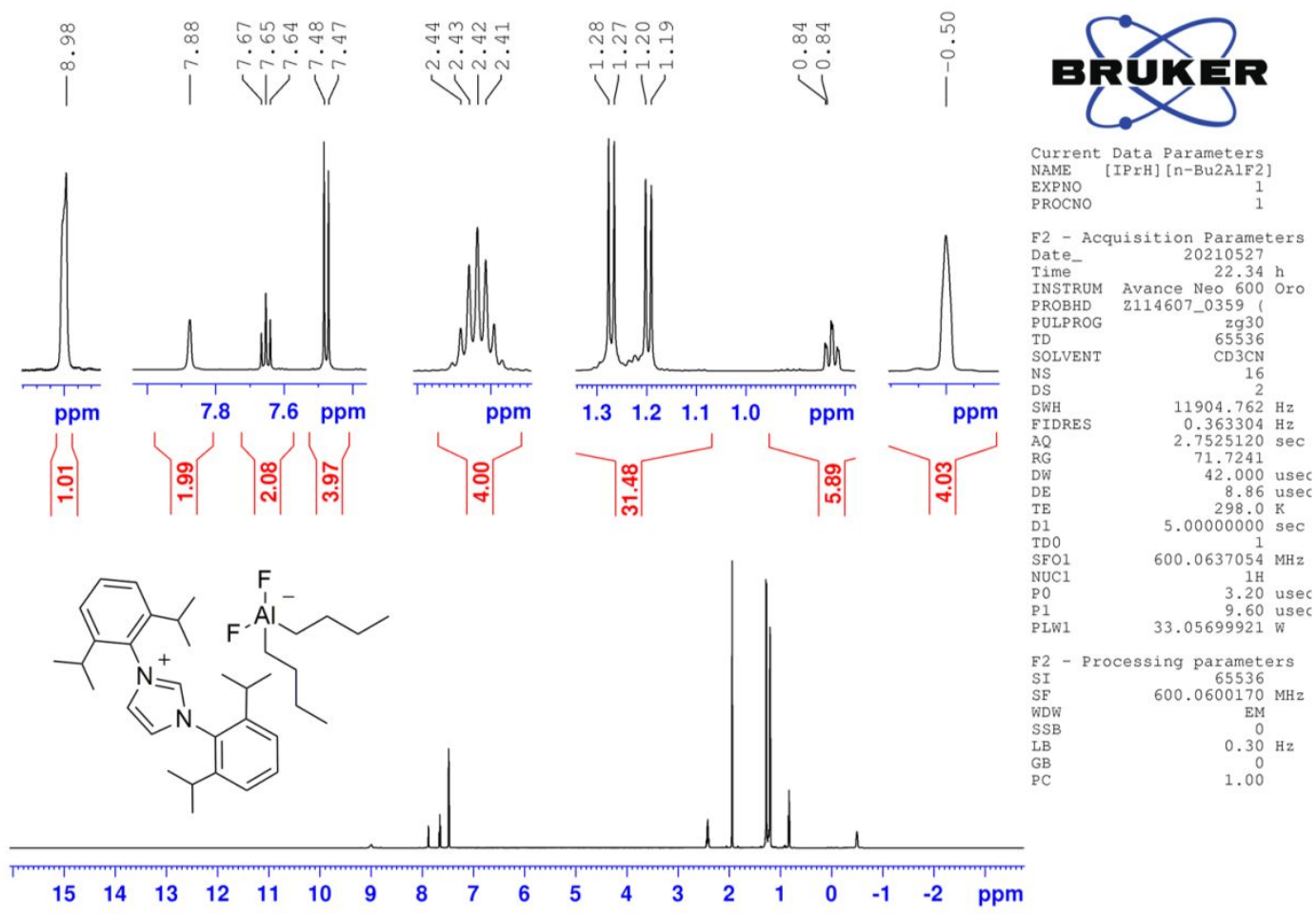

Figure S29. ${ }^{1} \mathrm{H}$ NMR spectrum of $[\mathrm{IPrH}]\left[n-\mathrm{Bu}_{2} \mathrm{AlF}_{2}\right](6)$ in acetonitrile solution.

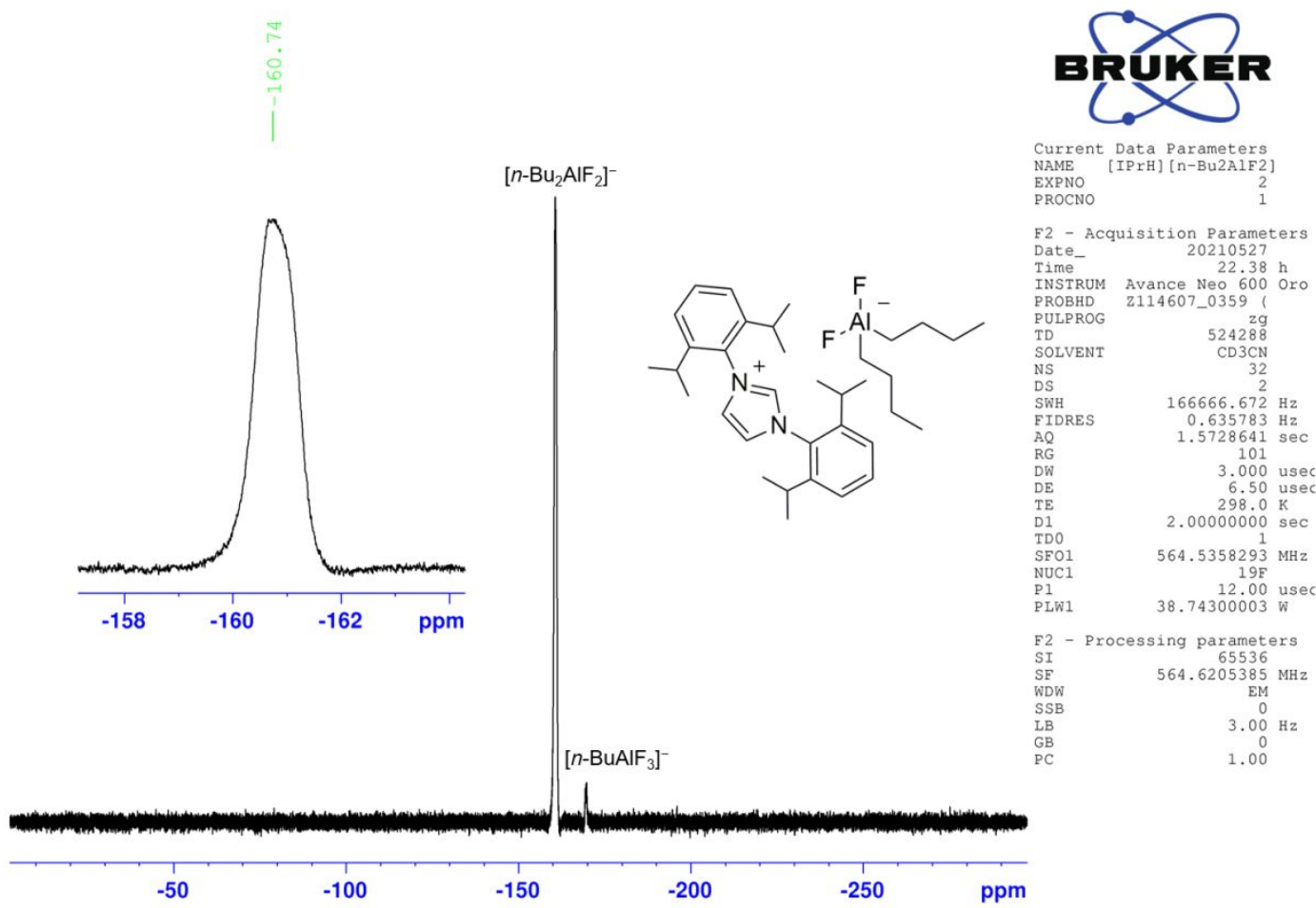

Figure S30. ${ }^{19} \mathrm{~F}$ NMR spectrum of $[\mathrm{IPrH}]\left[n-\mathrm{Bu}_{2} \mathrm{AlF}_{2}\right](\mathbf{6})$ in acetonitrile solution. 


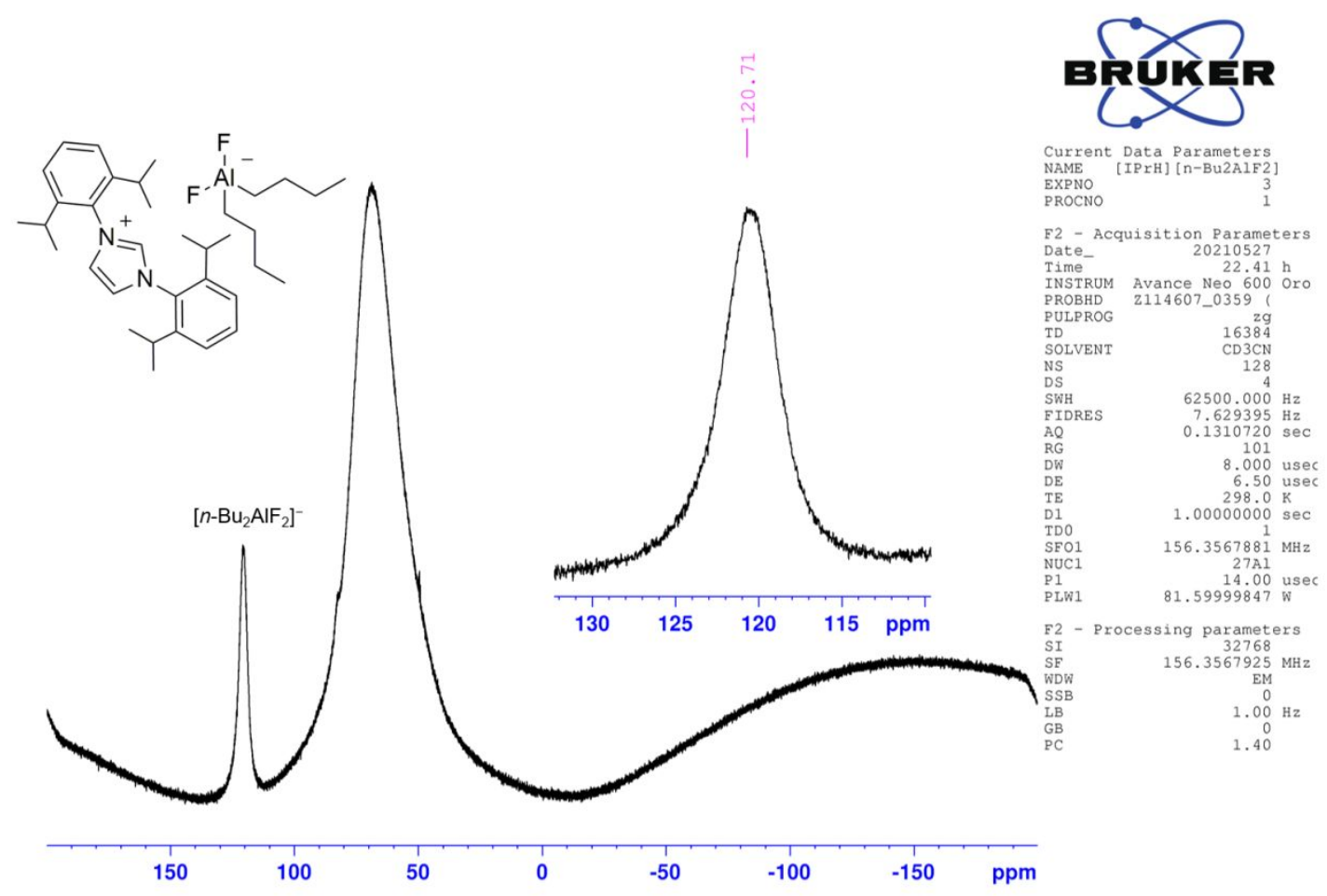

Figure S31. ${ }^{27} \mathrm{Al} \mathrm{NMR}$ spectrum of $[\mathrm{IPrH}]\left[n-\mathrm{Bu}_{2} \mathrm{AlF}_{2}\right](\mathbf{6})$ in acetonitrile solution.

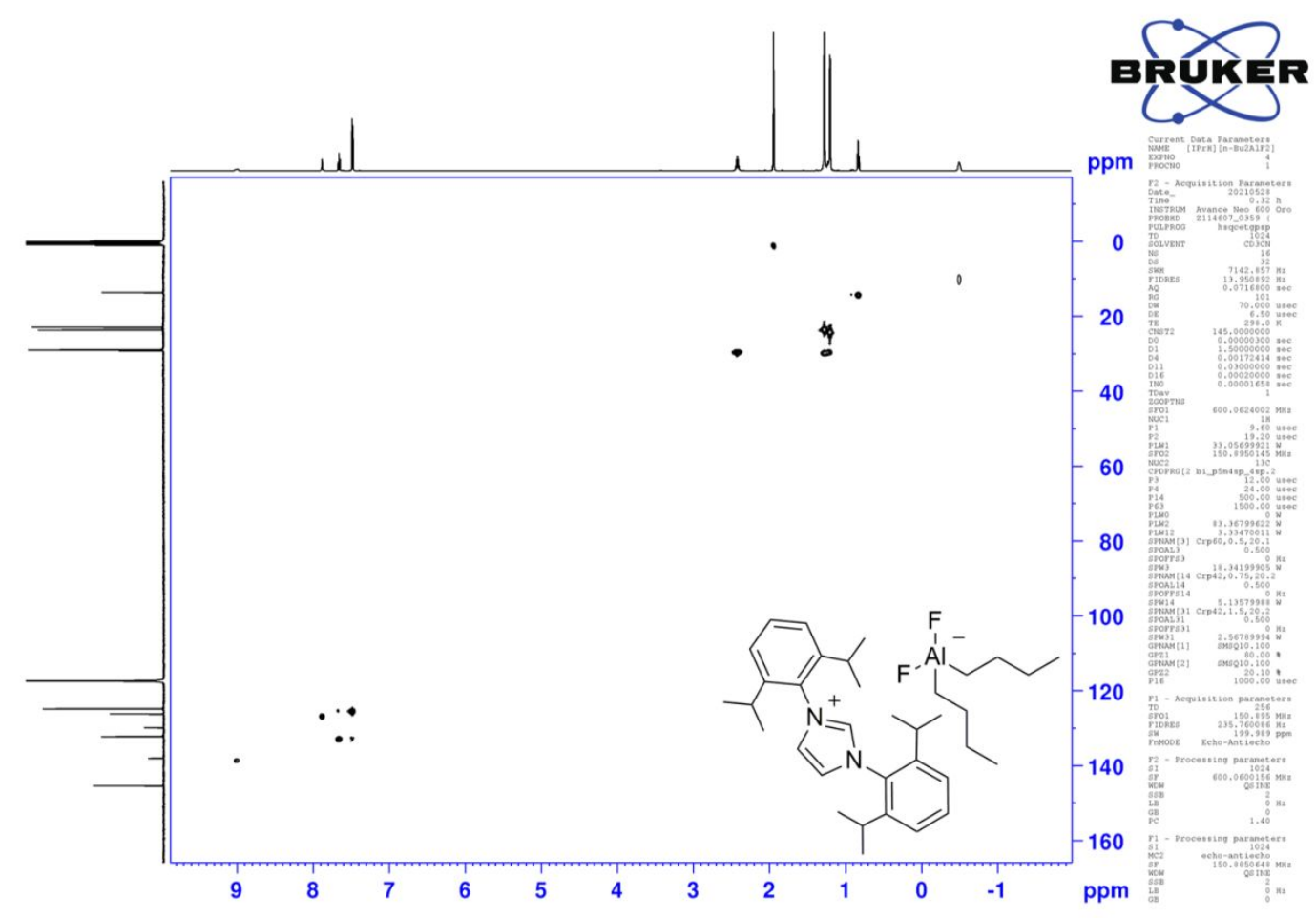

Figure S32. ${ }^{1} \mathrm{H}-{ }^{13} \mathrm{C}$ HSQC NMR spectrum of $[\mathrm{IPrH}]\left[n-\mathrm{Bu}_{2} \mathrm{AlF}_{2}\right](\mathbf{6})$ in acetonitrile solution. 
S1.11[IPrH]/n-BuAlF $]$ (7)

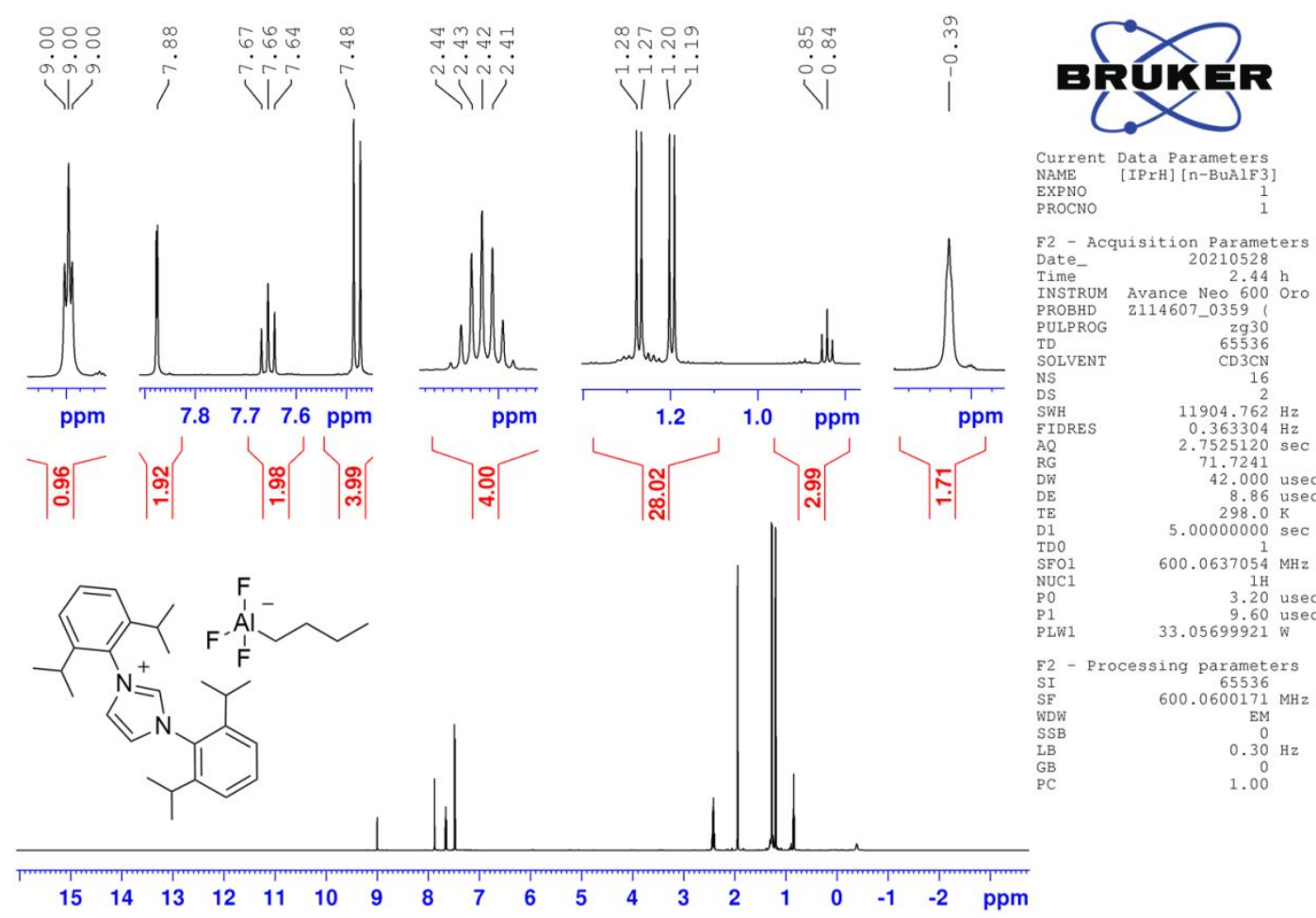

Figure S33. ${ }^{1} \mathrm{H}$ NMR spectrum of $[\mathrm{IPrH}]\left[n-\mathrm{BuAlF}_{3}\right](7)$ in acetonitrile solution.

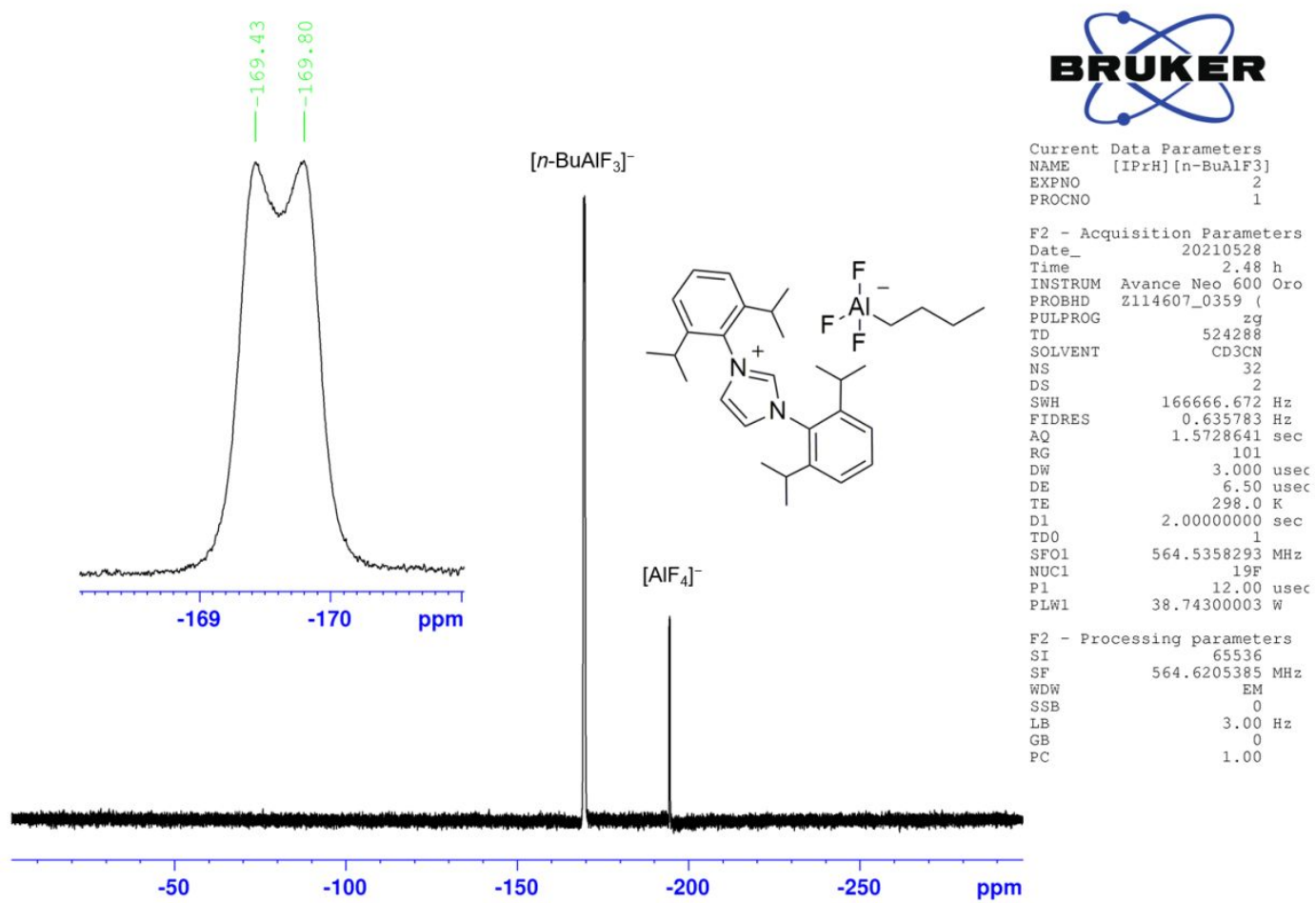

Figure S34. ${ }^{19} \mathrm{~F}$ NMR spectrum of $[\mathrm{IPrH}]\left[n-\mathrm{BuAlF}_{3}\right](7)$ in acetonitrile solution. 


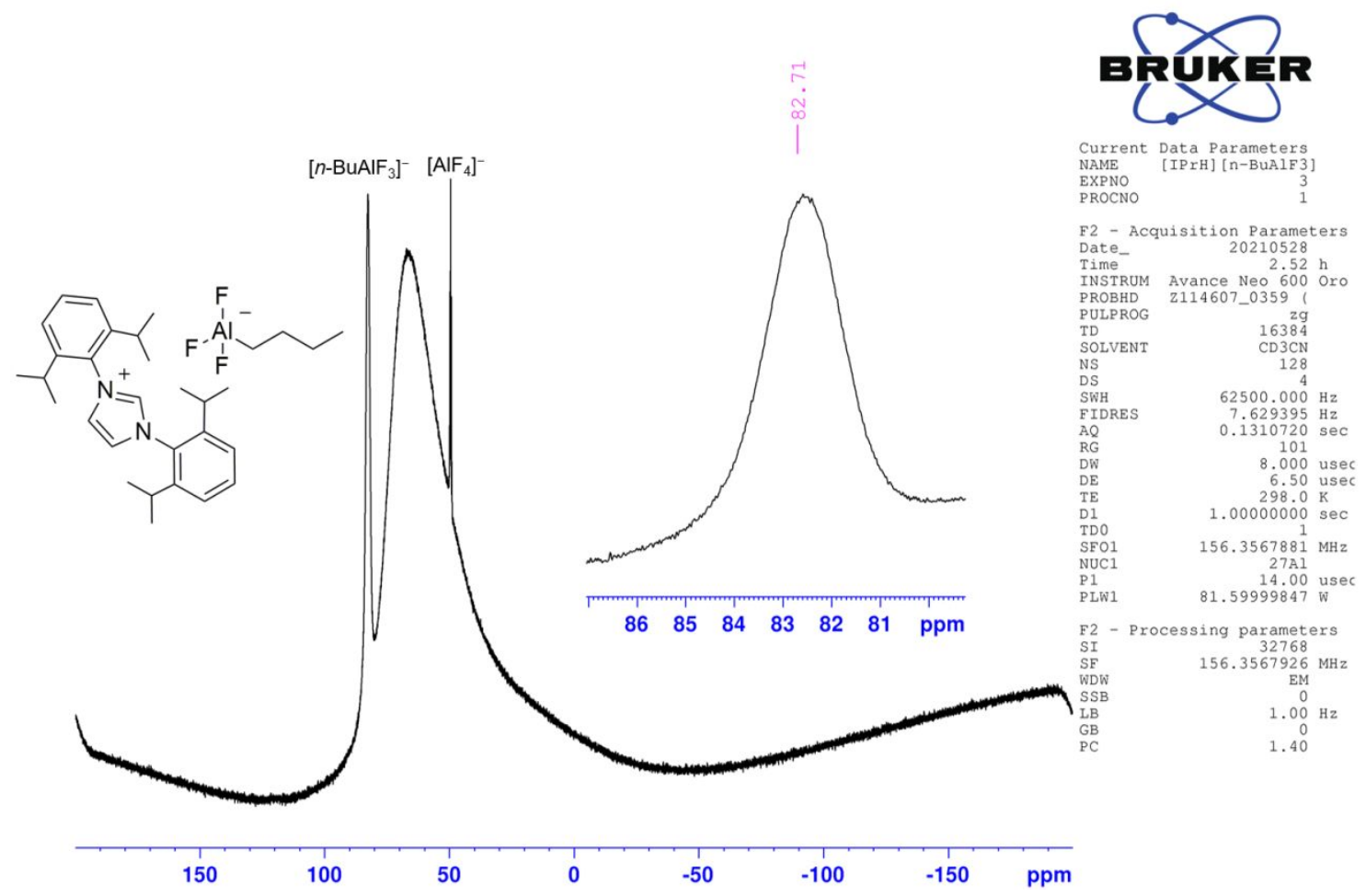

Figure S35. ${ }^{27} \mathrm{Al}$ NMR spectrum of $[\mathrm{IPrH}]\left[n-\mathrm{BuAlF}_{3}\right](7)$ in acetonitrile solution.

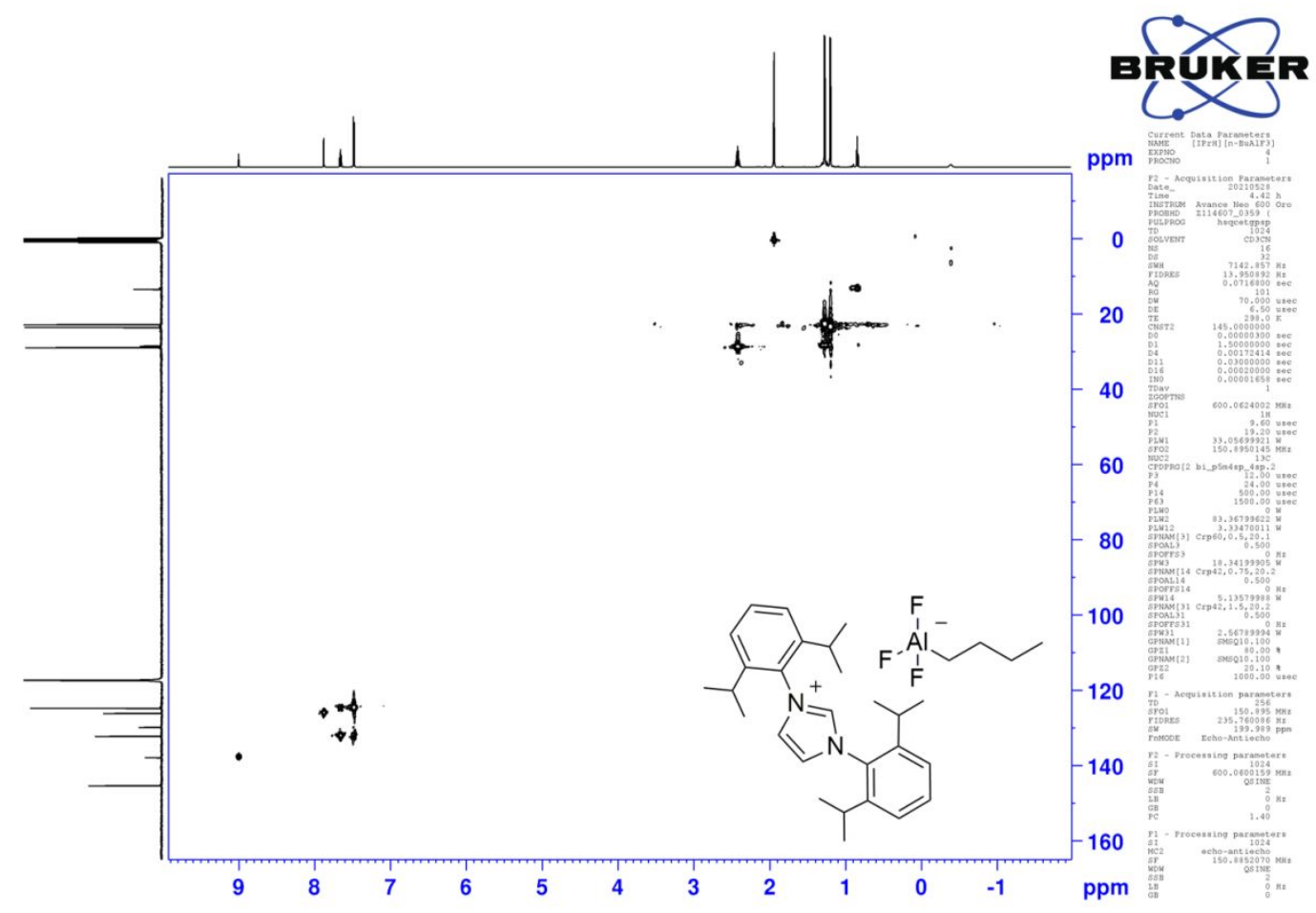

Figure S36. ${ }^{1} \mathrm{H}-{ }^{13} \mathrm{C}$ HSQC NMR spectrum of $[\mathrm{IPrH}]\left[n-\mathrm{BuAlF}_{3}\right](7)$ in acetonitrile solution. 


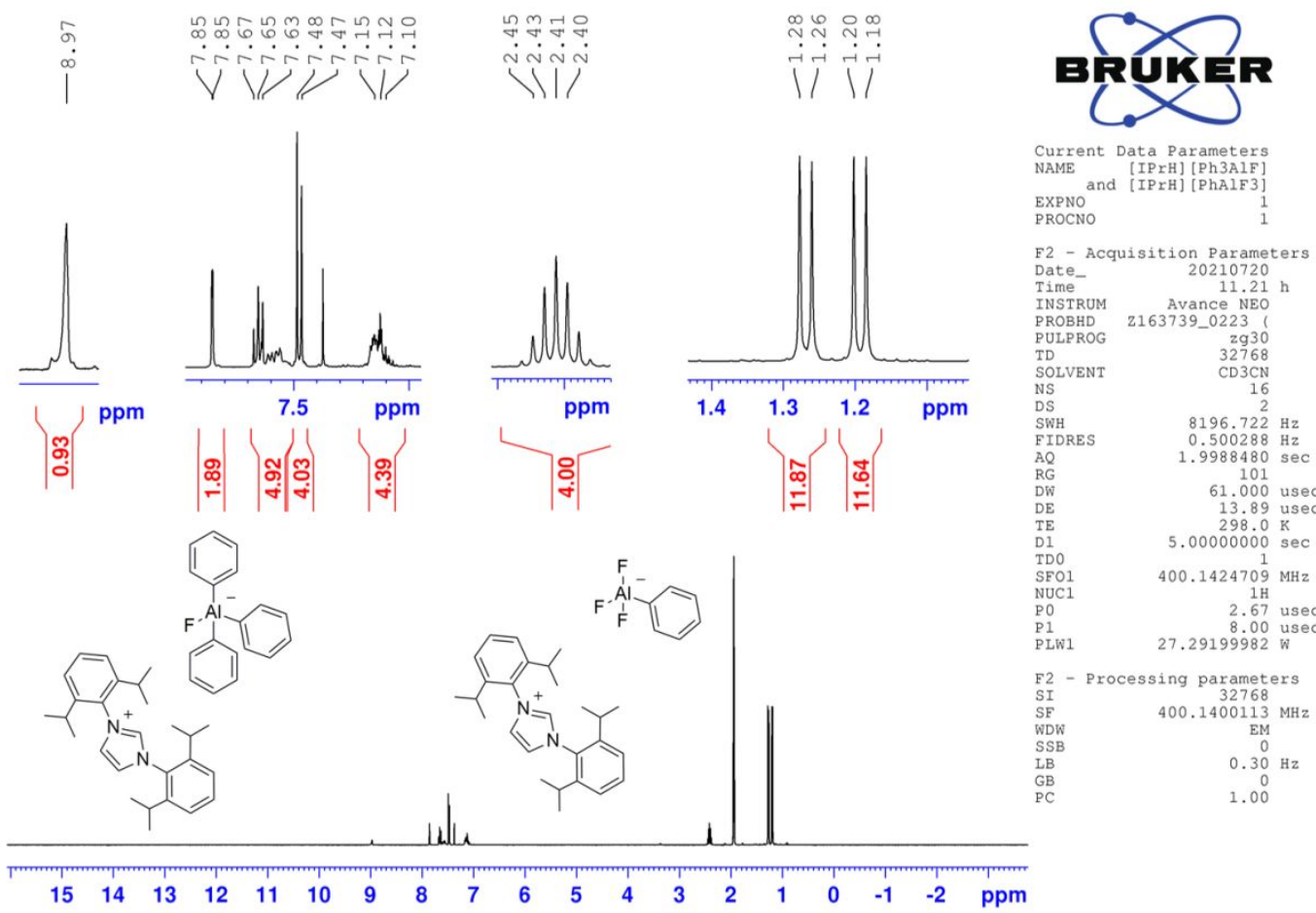

Figure S37. ${ }^{1} \mathrm{H}$ NMR spectrum of mixture of $[\mathrm{IPrH}]\left[\mathrm{Ph}_{3} \mathrm{AlF}\right](\mathbf{8})$ and $[\mathrm{IPrH}]\left[\mathrm{PhAlF}_{3}\right](\mathbf{1 0})$ in acetonitrile solution.

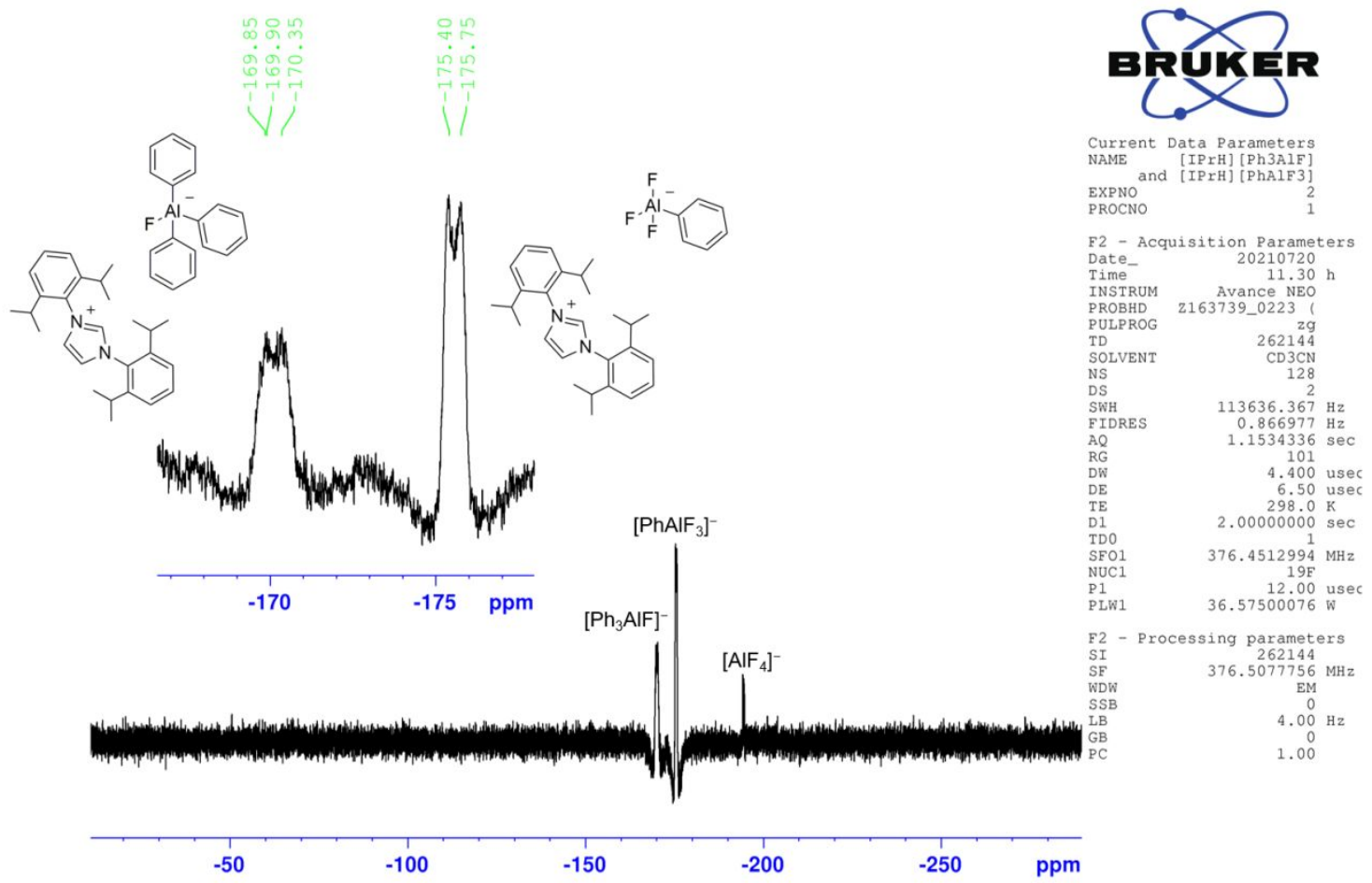

Figure S38. ${ }^{19} \mathrm{~F}$ NMR spectrum of mixture of $[\mathrm{IPrH}]\left[\mathrm{Ph}_{3} \mathrm{AlF}\right](\mathbf{8})$ and $[\mathrm{IPrH}]\left[\mathrm{PhAlF}_{3}\right](\mathbf{1 0})$ in acetonitrile solution. 


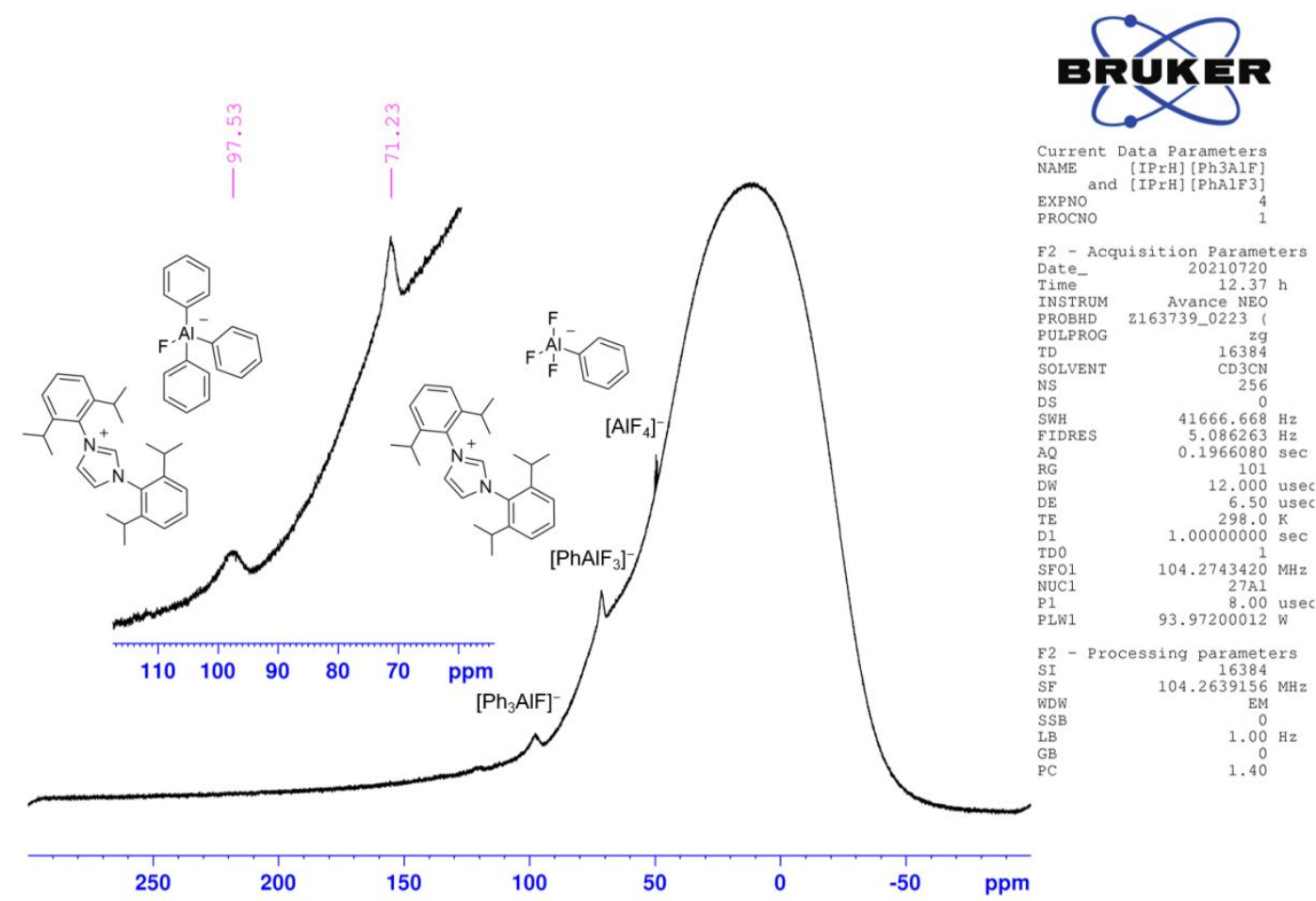

Figure S39. ${ }^{27} \mathrm{Al}$ NMR spectrum of mixture of $[\mathrm{IPrH}]\left[\mathrm{Ph}_{3} \mathrm{AlF}\right](\mathbf{8})$ and $[\mathrm{IPrH}]\left[\mathrm{PhAlF}_{3}\right](\mathbf{1 0})$ in acetonitrile solution.

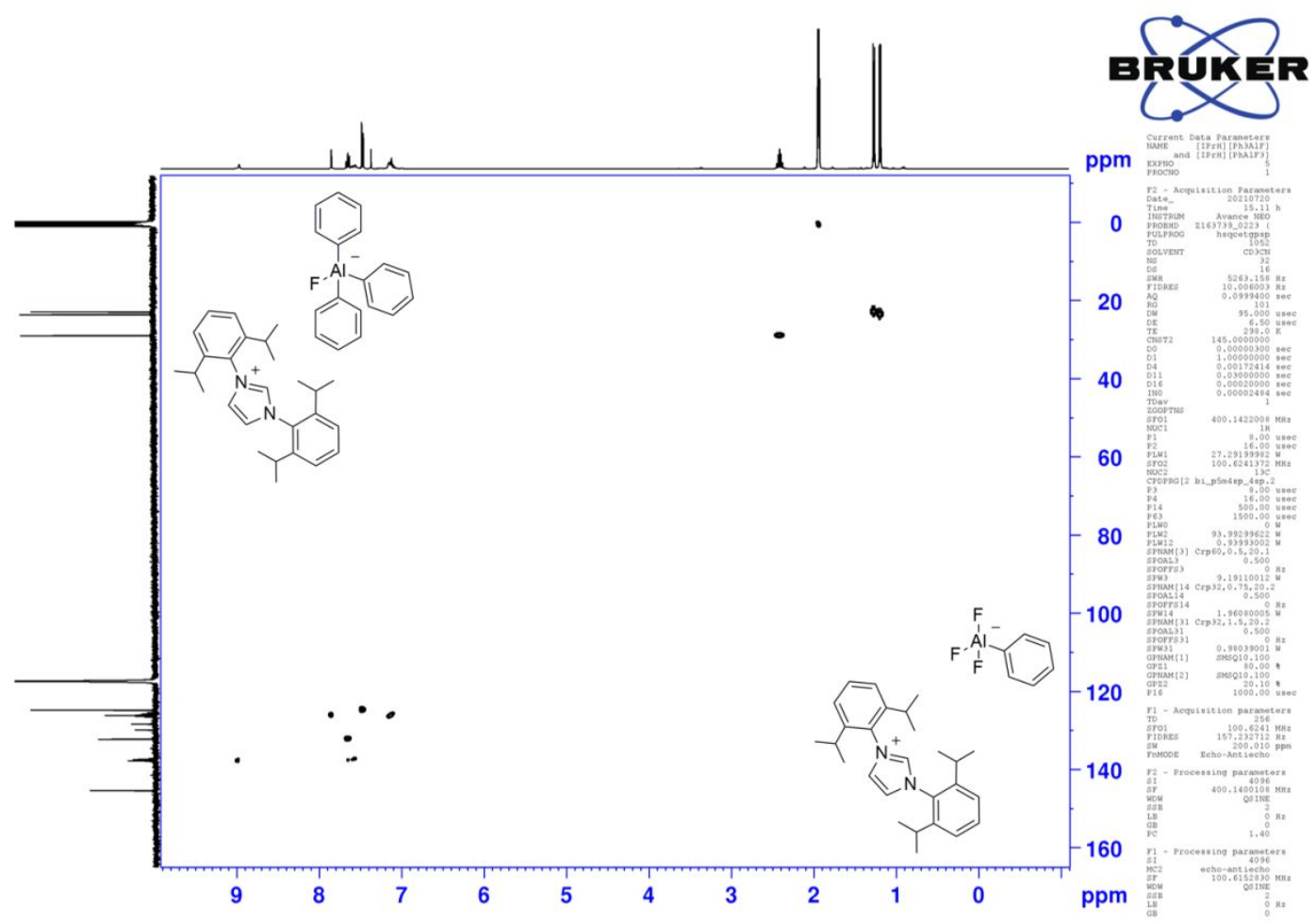

Figure S40. ${ }^{1} \mathrm{H}^{-13} \mathrm{C}$ HSQC NMR spectrum of mixture of $[\mathrm{IPrH}]\left[\mathrm{Ph}_{3} \mathrm{AlF}\right](\mathbf{8})$ and $[\mathrm{IPrH}]\left[\mathrm{PhAlF}_{3}\right](\mathbf{1 0})$ in acetonitrile solution. 


\section{S2 Raman spectroscopy}

S2.1 [IPrH][Me $\left.{ }_{4-n} \mathrm{AlF}_{n}\right]$, where $\mathrm{n}=1-4$

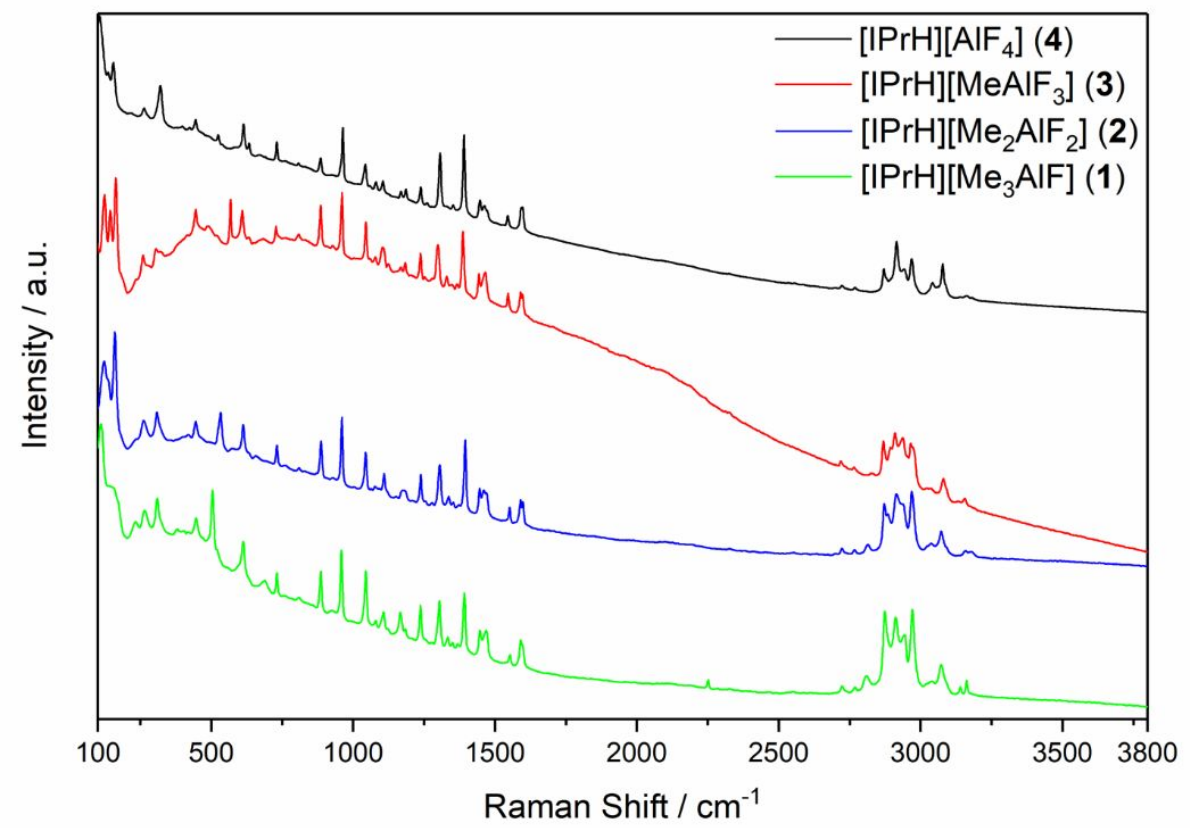

Figure S41. Raman spectra of [IPrH] $\left[\mathrm{Me}_{3} \mathrm{AlF}\right](\mathbf{1})$, [IPrH] $\left[\mathrm{Me}_{2} \mathrm{AlF}_{2}\right](\mathbf{2})$, [IPrH] $\left[\mathrm{MeAlF}_{3}\right](\mathbf{3})$ and [IPr] $\left.\mathrm{AlF}_{4}\right](\mathbf{4})$. 
S2.2 $[\mathrm{PrH}]\left[\mathrm{n}-\mathrm{B} \mathrm{u}_{4-n} A l F_{n}\right]$, where $\mathrm{n}=1-4$

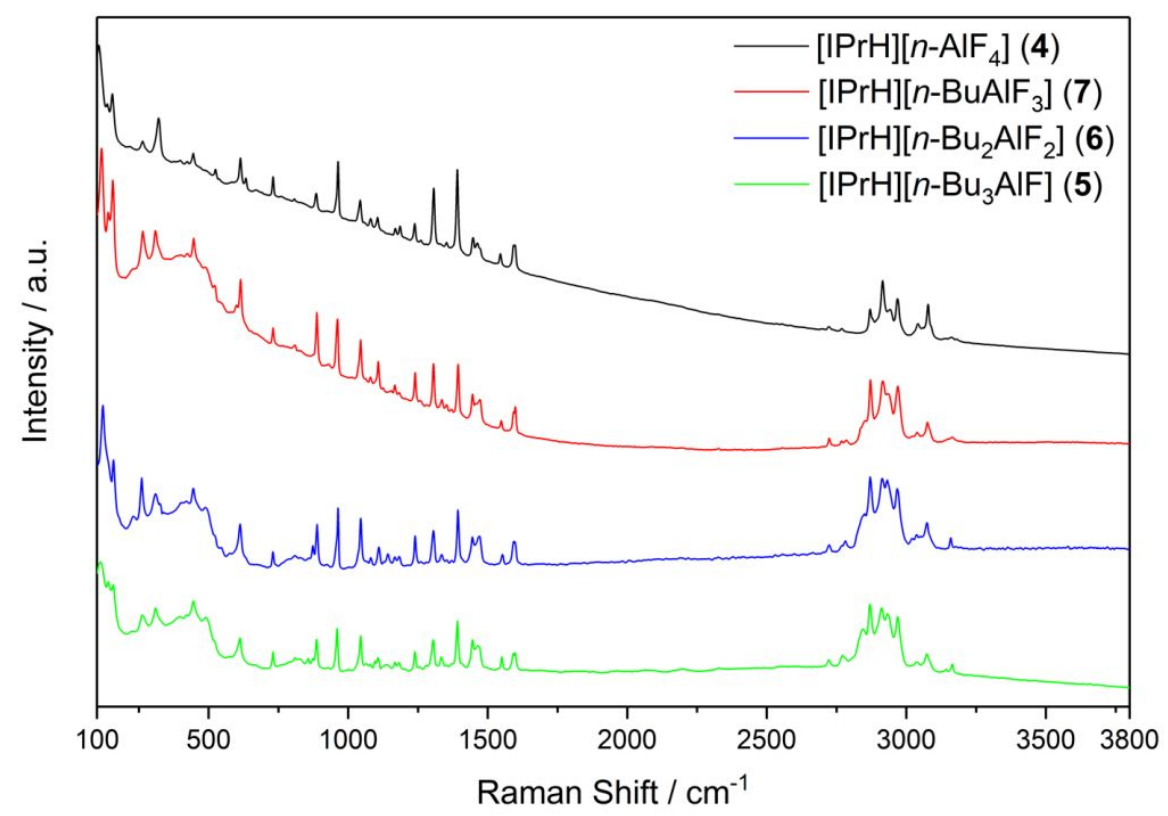

Figure S42. Raman spectra of [IPrH] $\left[n-\mathrm{Bu}_{3} \mathrm{AlF}\right](\mathbf{5})$, [IPrH $]\left[n-\mathrm{Bu}_{2} \mathrm{AlF}_{2}\right]$ (6), $[\mathrm{IPrH}][n-$ $\left.\mathrm{BuAlF}_{3}\right](7)$ and $[\mathrm{IPr}]\left[\mathrm{AlF}_{4}\right](\mathbf{4})$.

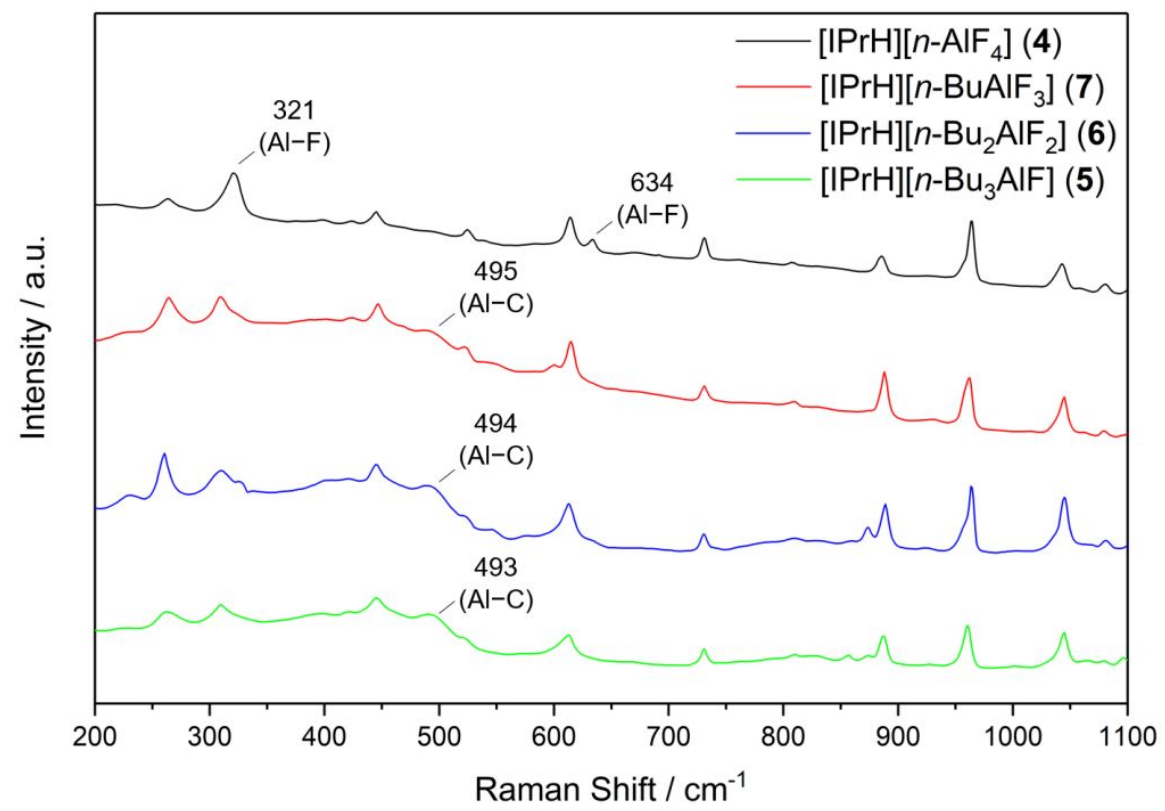

Figure S43. Raman spectra of $[\mathrm{IPrH}]\left[n-\mathrm{Bu}_{3} \mathrm{AlF}\right](\mathbf{5})$, [IPrH $]\left[n-\mathrm{Bu}_{2} \mathrm{AlF}_{2}\right]$ (6), [IPrH $][n-$ $\left.\mathrm{BuAlF}_{3}\right](7)$ and $[\mathrm{IPr}]\left[\mathrm{AlF}_{4}\right](4)$ with assigned bands for $\mathrm{Al}-\mathrm{F}$ and $\mathrm{Al}-\mathrm{C}$ vibrations. 
S2.3 $[\mathrm{IPrH}]\left[\mathrm{Ph}_{4-n} \mathrm{AlF}_{n}\right]$, where $\mathrm{n}=1-4$

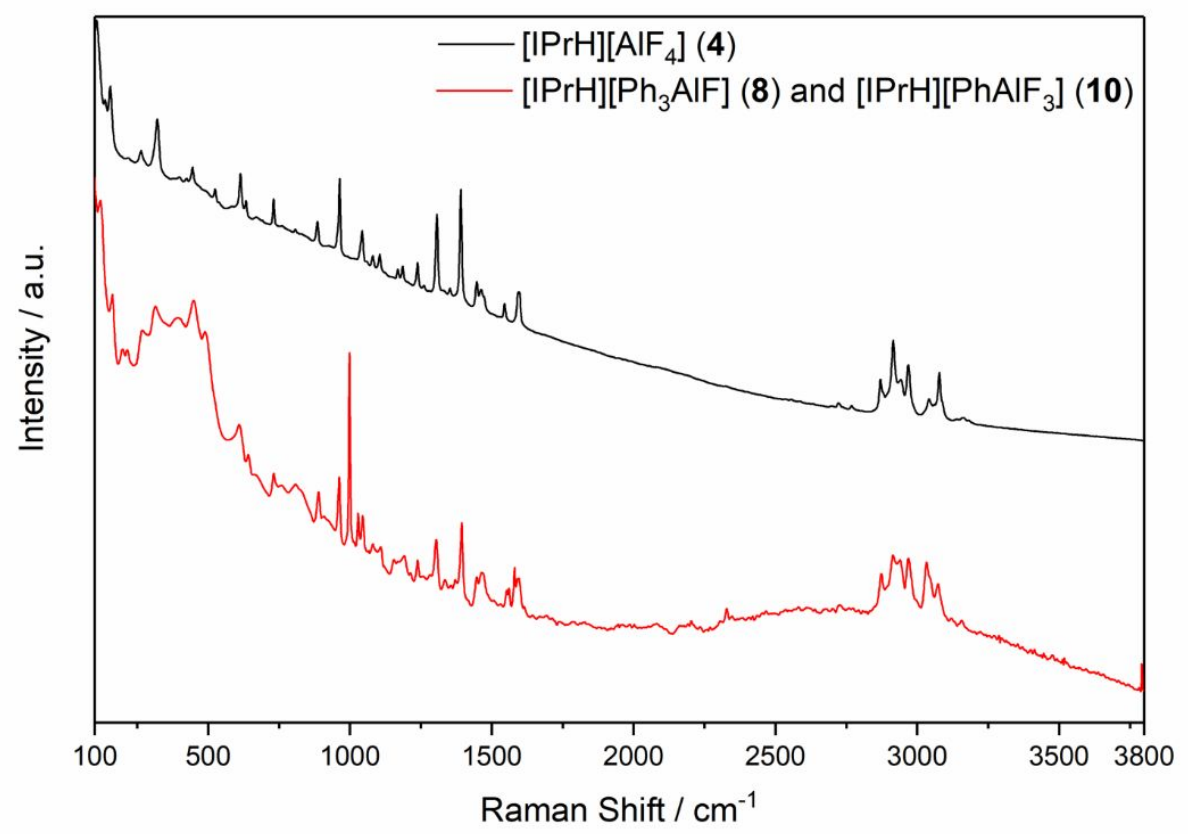

Figure S44. Raman spectra of mixture of $[\mathrm{IPrH}]\left[\mathrm{Ph}_{3} \mathrm{AlF}\right](\mathbf{8})$ and $[\mathrm{IPrH}]\left[\mathrm{PhAlF}_{3}\right](\mathbf{1 0})$.

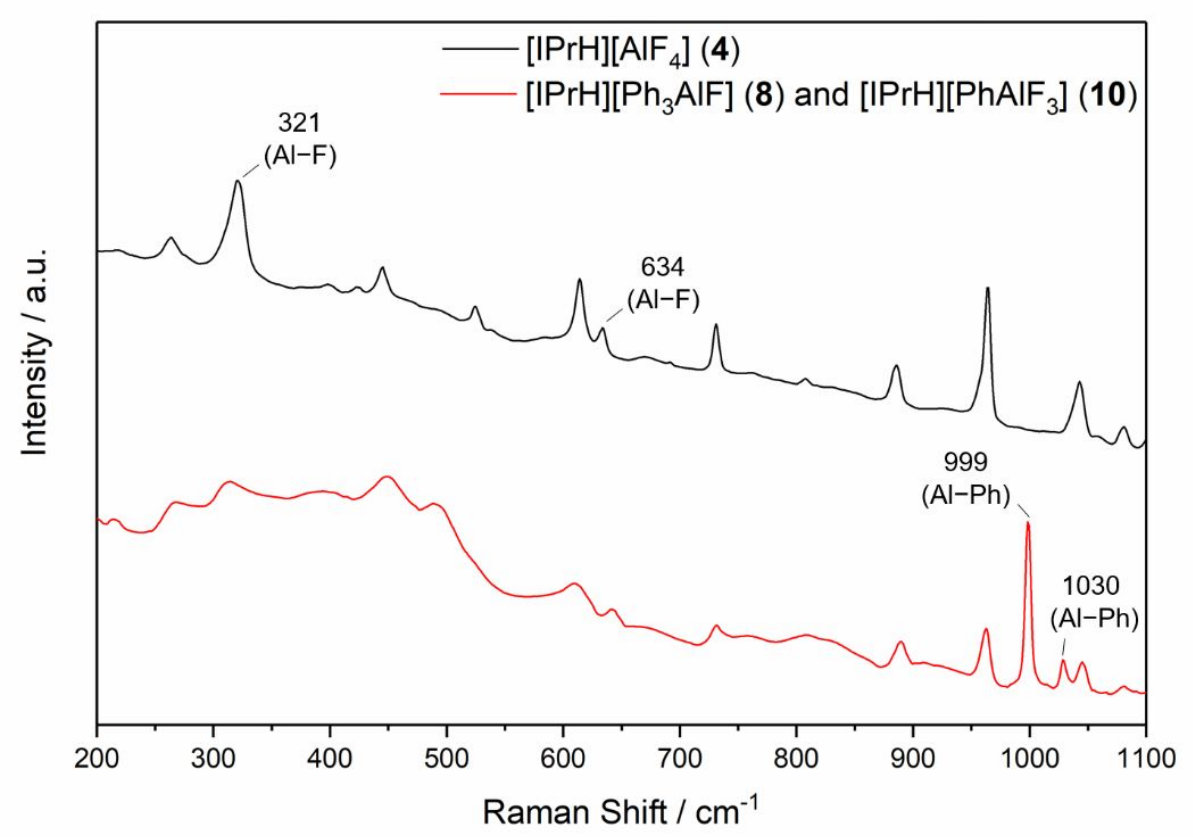

Figure S45. Raman spectra of mixture of $[\mathrm{IPrH}]\left[\mathrm{Ph}_{3} \mathrm{AlF}\right](\mathbf{8})$ and $[\mathrm{IPrH}]\left[\mathrm{PhAlF}_{3}\right](\mathbf{1 0})$ with assigned bands for $\mathrm{Al}-\mathrm{F}$ and $\mathrm{Al}-\mathrm{C}$ vibrations. 


\section{S3 Computational results}

Table S1. Experimental and calculated bond distances of $[\mathrm{IPrH}]\left[n-\mathrm{Bu}_{4-\mathrm{n}} \mathrm{AlF}_{\mathrm{n}}\right]$, where $\mathrm{n}=1-4$.

\begin{tabular}{|c|c|c|c|c|}
\hline Compound & $d(\mathrm{Al}-\mathrm{C})(\AA)$ & & $d(\mathrm{Al}-\mathrm{F})(\AA)$ & \\
\hline & Experimental & Calculated & Experimental & Calculated \\
\hline$[\mathrm{IPrH}]\left[n-\mathrm{Bu}_{3} \mathrm{AlF}\right](\mathbf{5})$ & $\begin{array}{l}2.013(3) \\
1.994(3) \\
1.984(3)\end{array}$ & $\begin{array}{l}2.03 \\
2.02 \\
2.02\end{array}$ & $1.733(1)^{[\mathrm{a}]}$ & $1.81^{[\mathrm{a}]}$ \\
\hline$[\mathrm{IPrH}]\left[n-\mathrm{Bu}_{2} \mathrm{AlF}_{2}\right](\mathbf{6})$ & $\begin{array}{l}2.073(4) \\
1.980(2)\end{array}$ & $\begin{array}{l}2.01 \\
2.00\end{array}$ & $\begin{array}{l}1.7096(9)^{[\mathrm{a}]} \\
1.7072(9)\end{array}$ & $\begin{array}{l}1.78^{[\mathrm{a}]} \\
1.71\end{array}$ \\
\hline$[\mathrm{IPrH}]\left[n-\mathrm{BuAlF}_{3}\right](\mathbf{7})$ & $1.923(18)$ & 1.98 & $\begin{array}{l}1.539(4)^{[\mathrm{b}]} \\
1.592(5)^{[\mathrm{b}]}\end{array}$ & $\begin{array}{l}1.75^{[\mathrm{a}]} \\
1.74 \\
1.71\end{array}$ \\
\hline$[\mathrm{IPrH}]\left[\mathrm{AlF}_{4}\right](4)$ & - & - & $\begin{array}{l}1.669(1) \\
1.660(1)\end{array}$ & $\begin{array}{l}1.73 \\
1.73 \\
1.68 \\
1.68\end{array}$ \\
\hline
\end{tabular}

[a] Al-F distances, where fluorine atoms form the strongest hydrogen bonds with hydrogen atoms on $\mathrm{C} 2$ position. [b] $\mathrm{Al}-\mathrm{F}$ distances reported for domain $\mathrm{A}$, where fluorine atoms are disordered.

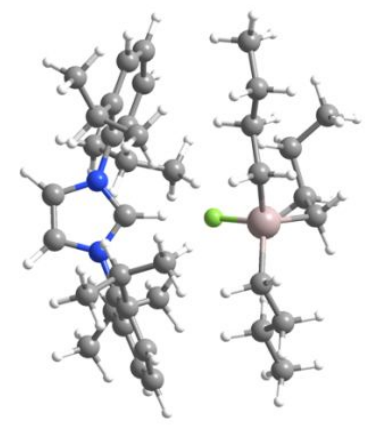

$[\mathrm{IPrH}]\left[n-\mathrm{Bu}_{3} \mathrm{AIF}\right](\mathbf{5})$

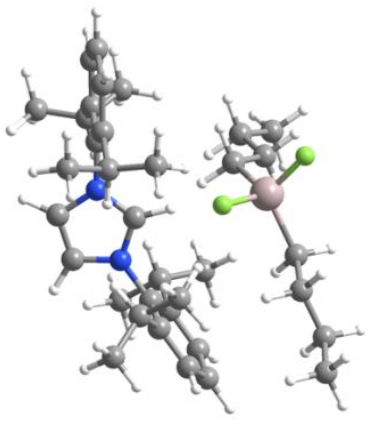

$[\mathrm{IPrH}]\left[n-\mathrm{Bu}_{2} \mathrm{AlF}_{2}\right](\mathbf{6})$

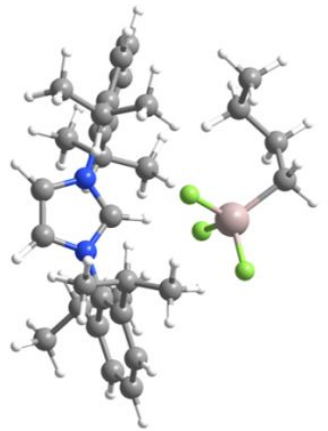

$[\mathrm{IPrH}]\left[n-\mathrm{BuAlF}_{3}\right](\mathbf{7})$

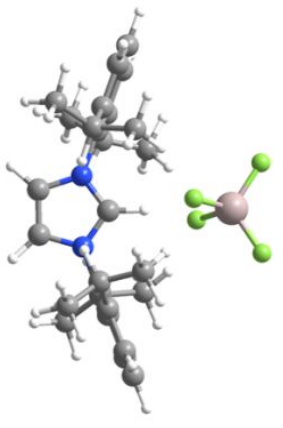

$[\mathrm{IPrH}]\left[\mathrm{AlF}_{4}\right](\mathbf{4})$

Figure S46. Optimized structures of $[\mathrm{IPrH}]\left[n-\mathrm{Bu}_{4-\mathrm{n}} \mathrm{AlF}_{\mathrm{n}}\right]$, where $\mathrm{n}=1-4$. 
Table S2. Experimental and calculated bond distances of $[\mathrm{IPrH}]\left[\mathrm{Ph}_{4-\mathrm{n}} \mathrm{AlF}_{\mathrm{n}}\right]$, where $\mathrm{n}=1-4$.

\begin{tabular}{|c|c|c|c|c|}
\hline Compound & \multicolumn{2}{|l|}{$d(\mathrm{Al}-\mathrm{C})(\AA)$} & \multicolumn{2}{|l|}{$d(\mathrm{Al}-\mathrm{F})(\AA)$} \\
\hline & Experimental & Calculated & Experimental & Calculated \\
\hline$[\mathrm{IPrH}]\left[\mathrm{Ph}_{3} \mathrm{AlF}\right](\mathbf{8})$ & $\begin{array}{l}2.004(2) \\
2.004(2) \\
2.000(2) \\
\end{array}$ & $\begin{array}{l}2.03 \\
2.00 \\
2.00\end{array}$ & $1.730(1)^{[\mathrm{a}]}$ & $1.77^{[\mathrm{a}]}$ \\
\hline$[\mathrm{IPrH}]\left[\mathrm{Ph}_{2} \mathrm{AlF}_{2}\right](\mathbf{9})$ & n.a. & $\begin{array}{l}1.98 \\
1.99 \\
\end{array}$ & n.a. & $\begin{array}{l}1.76^{[\mathrm{a}]} \\
1.75\end{array}$ \\
\hline$[\mathrm{IPrH}]\left[\mathrm{PhAlF}{ }_{3}\right](\mathbf{1 0})$ & $1.960(3)$ & 1.97 & $\begin{array}{l}1.683(2) \\
2.155(4)^{[b]} \\
1.703(6)^{[b]}\end{array}$ & $\begin{array}{l}1.74^{[\mathrm{a}]} \\
1.74 \\
1.69\end{array}$ \\
\hline$[\mathrm{IPrH}]\left[\mathrm{AlF}_{4}\right](\mathbf{4})$ & - & - & $\begin{array}{l}1.669(1) \\
1.660(1)\end{array}$ & $\begin{array}{l}1.73 \\
1.73 \\
1.68 \\
1.68\end{array}$ \\
\hline
\end{tabular}

[a] Al-F distances, where fluorine atoms form the strongest hydrogen bonds with hydrogen atoms on $\mathrm{C} 2$ position are written in italics. [b] $\mathrm{Al}-\mathrm{F}$ distances reported for domain $\mathrm{A}$, where fluorine atoms are disordered.

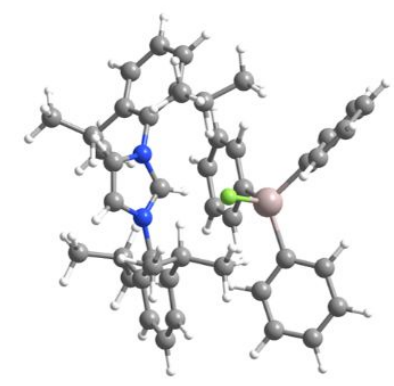

$[\mathrm{IPrH}]\left[\mathrm{Ph}_{3} \mathrm{AIF}\right](8)$

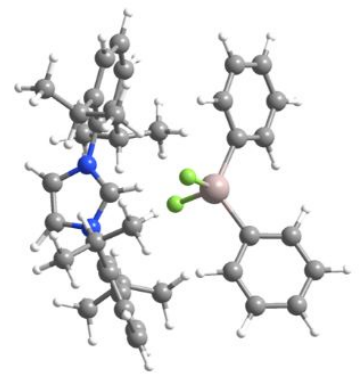

$[\mathrm{IPrH}]\left[\mathrm{Ph}_{2} \mathrm{AlF}_{2}\right](\mathbf{9})$

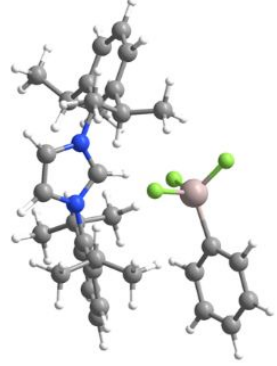

$[\mathrm{IPrH}]\left[\mathrm{PhAlF}_{3}\right](\mathbf{1 0})$

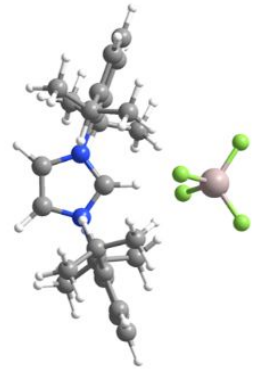

$[\mathrm{IPrH}]\left[\mathrm{AlF}_{4}\right](\mathbf{4})$

Figure S47. Optimized structures of [IPrH] $\left[\mathrm{Ph}_{4-n} \mathrm{AlF}_{n}\right]$, where $\mathrm{n}=1-4$. 
Table S3. Calculated electronic energies $(E)$ at the PBE/def2TZVP level of theory and calculated energies of reactions $\Delta E$ in a.u. and $\mathrm{kJ} / \mathrm{mol}$.

\begin{tabular}{|c|c|c|c|c|}
\hline Compound & $E /$ a.u. & Reaction & $\Delta E /$ a.u. & $\begin{array}{l}\Delta E / \\
\mathrm{kJ} / \mathrm{mol}\end{array}$ \\
\hline [IPrH][F] & -1259.411123 & - & - & (- \\
\hline $\mathrm{HF}$ & -100.3913792 & - & - & - \\
\hline $\mathrm{AlMe}_{3}$ & -361.9220025 & - & - & 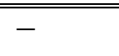 \\
\hline $\mathrm{Al}(n-\mathrm{Bu})_{3}$ & -715.3628002 & - & - & - \\
\hline $\mathrm{AlPh}_{3}$ & -936.6345615 & - & - & - \\
\hline$\overline{\mathrm{CH}_{4}}$ & -40.46417895 & $\overline{-1-}$ & $\overline{-1}$ & $\overline{-1-}$ \\
\hline$n-\mathrm{BuH}$ & -158.2801283 & - & - & - \\
\hline $\mathrm{PhH}$ & -232.0300314 & - & - & - \\
\hline 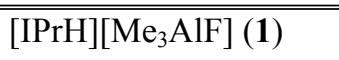 & -1621.399002 & $=[\mathrm{IIPrH}][\mathrm{F}]+\mathrm{AlMe}_{3} \rightarrow[\mathrm{IPrH}]\left[\mathrm{Me}_{3} \mathrm{AlF}\right]$ & "-0.0658759 & 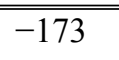 \\
\hline$[\mathrm{IPrH}]\left[\mathrm{Me}_{2} \mathrm{AlF}_{2}\right](2)$ & -1681.422418 & $\begin{array}{l}{[\mathrm{IPrH}][\mathrm{F}]+\mathrm{HF}+\mathrm{AlMe}_{3} \rightarrow} \\
{[\mathrm{IPrH}]\left[\mathrm{Me}_{2} \mathrm{AlF}_{2}\right]+\mathrm{CH}_{4}}\end{array}$ & -0.1620916 & -426 \\
\hline$[\mathrm{IPrH}]\left[\mathrm{MeAlF}_{3}\right](\mathbf{3})$ & -1741.432198 & 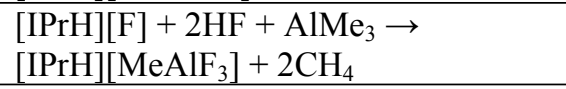 & -0.2446717 & -642 \\
\hline$[\mathrm{IPrH}]\left[\mathrm{AlF}_{4}\right](4)$ & -1801.432451 & $\begin{array}{l}{[\mathrm{IPrH}][\mathrm{F}]+3 \mathrm{HF}+\mathrm{AlMe}_{3} \rightarrow} \\
{[\mathrm{IPrH}]\left[\mathrm{AlF}_{4}\right]+3 \mathrm{CH}_{4}}\end{array}$ & -0.3177247 & -834 \\
\hline$\left[(\mathrm{IPr}) \mathrm{AlMe}_{3}\right]$ & -1520.964628 & $\begin{array}{l}2[\mathrm{IPrH}][\mathrm{F}]+2 \mathrm{AlMe}_{3} \rightarrow \\
{[\mathrm{IPrH}]\left[\mathrm{Me}_{2} \mathrm{AlF}_{2}\right]+\left[(\mathrm{IPr}) \mathrm{AlMe}_{3}\right]+} \\
\mathrm{CH}_{4}\end{array}$ & -0.1849731 & -486 \\
\hline $2[\mathrm{IPrH}]\left[\mathrm{Me}_{3} \mathrm{AlF}\right](\mathbf{1})$ & -3242.798003 & $\begin{array}{l}2[\mathrm{IPrH}][\mathrm{F}]+2 \mathrm{AlMe}_{3} \rightarrow \\
2[\mathrm{IPrH}]\left[\mathrm{Me}_{3} \mathrm{AlF}\right]\end{array}$ & -0.13175173 & -346 \\
\hline$[\mathrm{IPrH}]\left[n-\mathrm{Bu}_{3} \mathrm{AlF}\right](\mathbf{5})$ & -1974.850042 & $\begin{array}{l}\mathrm{IPrH}][\mathrm{F}]+\mathrm{Al}(n-\mathrm{Bu})_{3} \rightarrow \\
{[\mathrm{IPrH}]\left[n-\mathrm{Bu}_{3} \mathrm{AlF}\right]}\end{array}$ & -0.0761185 & -200 \\
\hline$[\mathrm{IPrH}]\left[n-\mathrm{Bu}_{2} \mathrm{AlF}_{2}\right](\mathbf{6})$ & -1917.046026 & $\begin{array}{l}{[\mathrm{IPrH}][\mathrm{F}]+\mathrm{HF}+\mathrm{Al}(n-\mathrm{Bu})_{3} \rightarrow} \\
{[\mathrm{IPrH}]\left[n-\mathrm{Bu}_{2} \mathrm{AlF}_{2}\right]+n-\mathrm{BuH}}\end{array}$ & -0.1608517 & -422 \\
\hline$[\mathrm{IPrH}]\left[n-\mathrm{BuAlF}{ }_{3}\right](7)$ & -1859.249810 & $\begin{array}{l}{[\mathrm{IPrH}][\mathrm{F}]+2 \mathrm{HF}+\mathrm{Al}(n-\mathrm{Bu})_{3} \rightarrow} \\
{[\mathrm{IPrH}]\left[n-\mathrm{BuAlF}{ }_{3}\right]+2 n-\mathrm{BuH}}\end{array}$ & -0.2533845 & -665 \\
\hline$[\mathrm{IPrH}]\left[\mathrm{AlF}_{4}\right](4)$ & -1801.432451 & $\begin{array}{l}{[\mathrm{IPrH}][\mathrm{F}]+3 \mathrm{HF}+\mathrm{Al}(n-\mathrm{Bu})_{3} \rightarrow} \\
{[\mathrm{IPrH}]\left[\mathrm{AlF}_{4}\right]+3 n-\mathrm{BuH}}\end{array}$ & -0.3247751 & -853 \\
\hline$\left[(\operatorname{IPr}) \mathrm{Al}(n-\mathrm{Bu})_{3}\right]$ & -1874.409759 & $\begin{array}{l}2[\mathrm{IPrH}][\mathrm{F}]+2 \mathrm{Al}(n-\mathrm{Bu})_{3} \rightarrow \\
{[\mathrm{IPrH}]\left[n-\mathrm{Bu}_{2} \mathrm{AlF}_{2}\right]+\left[(\mathrm{IPr}) \mathrm{Al}(n-\mathrm{Bu})_{3}\right]} \\
+n-\mathrm{BuH}\end{array}$ & -0.1880667 & -494 \\
\hline $2[\mathrm{IPrH}]\left[n-\mathrm{Bu}_{3} \mathrm{AlF}\right](\mathbf{5})$ & -3949.700084 & $\begin{array}{l}2[\mathrm{IPrH}][\mathrm{F}]+2 \mathrm{Al}(n-\mathrm{Bu})_{3} \rightarrow \\
2[\mathrm{IPrH}]\left[n-\mathrm{Bu}_{3} \mathrm{AlF}\right]\end{array}$ & -0.15223709 & -400 \\
\hline$[\mathrm{IPrH}]\left[\mathrm{Ph}_{3} \mathrm{AlF}\right](\mathbf{8})$ & -2196.136413 & {$[\mathrm{EIPrH}][\mathrm{F}]+\mathrm{AlPh}_{3} \rightarrow[\mathrm{IPrH}]\left[\mathrm{Ph}_{3} \mathrm{AlF}\right]$} & "-0.0907278 & -238 \\
\hline$[\mathrm{IPrH}]\left[\mathrm{Ph}_{2} \mathrm{AlF}_{2}\right](\mathbf{9})$ & -2064.572196 & $\begin{array}{l}{[\mathrm{IPrH}][\mathrm{F}]+\mathrm{HF}+\mathrm{AlPh}_{3} \rightarrow} \\
{[\mathrm{IPrH}]\left[\mathrm{Ph}_{2} \mathrm{AlF}_{2}\right]+\mathrm{C}_{6} \mathrm{H}_{6}}\end{array}$ & -0.1651634 & -434 \\
\hline$[\mathrm{IPrH}]\left[\mathrm{PhAlF}{ }_{3}\right](\mathbf{1 0})$ & -1933.006025 & $\begin{array}{l}{[\mathrm{IPrH}][\mathrm{F}]+2 \mathrm{HF}+\mathrm{AlPh}_{3} \rightarrow} \\
{[\mathrm{IPrH}]\left[\mathrm{PhAlF} \mathrm{Ph}_{3}\right]+2 \mathrm{C}_{6} \mathrm{H}_{6}}\end{array}$ & -0.2376442 & -624 \\
\hline$[\mathrm{IPrH}]\left[\mathrm{AlF}_{4}\right](4)$ & -1801.432451 & $\begin{array}{l}{[\mathrm{IPrH}][\mathrm{F}]+3 \mathrm{HF}+\mathrm{AlPh}_{3} \rightarrow} \\
{[\mathrm{IPrH}]\left[\mathrm{AlF}_{4}\right]+3 \mathrm{C}_{6} \mathrm{H}_{6}}\end{array}$ & -0.3027230 & -795 \\
\hline$\left[(\mathrm{IPr}) \mathrm{AlPh}_{3}\right]$ & -2095.692660 & $\begin{array}{l}2[\mathrm{IPrH}][\mathrm{F}]+2 \mathrm{AlPh}_{3} \rightarrow \\
{[\mathrm{IPrH}]\left[\mathrm{Ph}_{2} \mathrm{AlF}_{2}\right]+\left[(\mathrm{IPr}) \mathrm{AlPh}_{3}\right]+\mathrm{C}_{6} \mathrm{D}_{6}}\end{array}$ & -0.2035181 & -534 \\
\hline $2[\mathrm{IPrH}]\left[\mathrm{Ph}_{3} \mathrm{AlF}\right](\mathbf{8})$ & $-4392,272825$ & $\begin{array}{l}2[\mathrm{IPrH}][\mathrm{F}]+2 \mathrm{AlPh}_{3} \rightarrow \\
2[\mathrm{IPrH}]\left[\mathrm{Ph}_{3} \mathrm{AlF}\right]\end{array}$ & $-0,18145565$ & -476 \\
\hline
\end{tabular}




\section{S3.1 DFT Calculation of Vibrational data}

We have performed DFT calculations of the anions to determine the positions of the peaks in the Raman spectra. We focused on the Raman active Al-F and $\mathrm{Al}-\mathrm{C}$ vibrations. The DFT calculations were performed as described in the experimental section and the results are shown in Table S4. The obtained values of the frequencies are higher when using the B3LYP potential compared with PBE and are closer to the experimental values. For all organofluoroaluminate anions, the intensities of the calculated Raman $\mathrm{Al}-\mathrm{F}$ and $\mathrm{Al}-\mathrm{C}$ vibrations were relatively low compared to the intensities of all the calculated data. This can be clearly seen from the graphical representation of all calculated IR and Raman frequencies in Figures S48, S49 and S50.

Table S4. Selected DFT calculated Raman frequencies at the PBE/def2TZVP and B3LYP/Def2TZVP level of theory. Only the frequencies with the highest Raman intensities that are related to the vibrations of $\mathrm{Al}-\mathrm{F}$ and $\mathrm{Al}-\mathrm{C}$ are presented and compared with experimentally obtained data.

\begin{tabular}{|c|c|c|c|}
\hline $\begin{array}{l}\text { Compound and } \\
\text { vibrations }\end{array}$ & $\begin{array}{l}\text { Raman freq. } / \mathrm{cm}^{-1} \\
\text { PBE/Def2TZVP }\end{array}$ & $\begin{array}{l}\text { Raman freq. / } \mathrm{cm}^{-1} \\
\text { B3LYP/Def2TZVP }\end{array}$ & $\begin{array}{c}\text { Raman freq. } / \mathrm{cm}^{-1} \\
\text { Experimental }\end{array}$ \\
\hline $\begin{array}{l}\left.\mathbf{A I F}_{4}\right]^{-} \\
v(\mathrm{Al}-\mathrm{F}) \\
v(\mathrm{Al}-\mathrm{F})\end{array}$ & $\begin{array}{l}292 \\
588\end{array}$ & $\begin{array}{l}303 \\
610\end{array}$ & $\begin{array}{l}321 \\
634\end{array}$ \\
\hline $\begin{array}{l}{\left[\mathbf{M e}_{3} \mathbf{A l F}\right]^{-}} \\
v(\mathrm{Al}-\mathrm{C}+\mathrm{Al}-\mathrm{F})\end{array}$ & 476 & 488 & 505 \\
\hline $\begin{array}{l}\left.\mathbf{I M e}_{\mathbf{2}} \mathbf{A l F} \mathbf{F}_{2}\right]^{-} \\
v(\mathrm{Al}-\mathrm{C}+\mathrm{Al}-\mathrm{F})\end{array}$ & 498 & 511 & 533 \\
\hline $\begin{array}{l}\left.\mathbf{M e A I F}_{3}\right]^{-} \\
v(\mathrm{Al}-\mathrm{C}+\mathrm{Al}-\mathrm{F})\end{array}$ & 534 & 549 & 567 \\
\hline 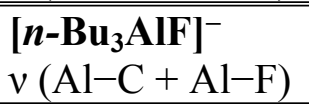 & 473 & 491 & 493 \\
\hline 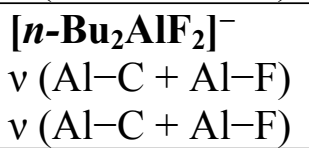 & $\begin{array}{l}395 \\
554\end{array}$ & $\begin{array}{l}408 \\
573\end{array}$ & 494 \\
\hline $\begin{array}{l}\left.\text { [n-BuAlF }_{3}\right]^{-} \\
v(\mathrm{Al}-\mathrm{C}+\mathrm{Al}-\mathrm{F}) \\
v(\mathrm{Al}-\mathrm{C}+\mathrm{Al}-\mathrm{F})\end{array}$ & $\begin{array}{l}441 \\
527\end{array}$ & $\begin{array}{l}457 \\
543\end{array}$ & 495 \\
\hline $\begin{array}{l}\left.\mathbf{P h}_{3} \mathbf{A l F}\right]^{-} \\
v(\mathrm{Al}-\mathrm{F}) \\
v(\mathrm{Al}-\mathrm{C}+\mathrm{Al}-\mathrm{F}) \\
v(\mathrm{Al}-\mathrm{C}+\mathrm{Al}-\mathrm{F})\end{array}$ & $\begin{array}{c}297 \\
636 \\
1019 \\
\end{array}$ & $\begin{array}{c}309 \\
663 \\
1049 \\
\end{array}$ & $999,1030^{[\mathrm{a}]}$ \\
\hline $\begin{array}{l}\left.\mathbf{P h}_{\mathbf{2}} \mathbf{A l F}_{\mathbf{2}}\right]^{-} \\
v(\mathrm{Al}-\mathrm{F}) \\
v(\mathrm{Al}-\mathrm{C}+\mathrm{Al}-\mathrm{F}) \\
v(\mathrm{Al}-\mathrm{C})\end{array}$ & $\begin{array}{c}331 \\
628 \\
1021 \\
\end{array}$ & $\begin{array}{c}345 \\
654 \\
1050 \\
\end{array}$ & \\
\hline $\begin{array}{l}\left.\mathbf{P h}_{\mathbf{P A l F}} \mathbf{A l}_{\mathbf{3}}\right]^{-} \\
v(\mathrm{Al}-\mathrm{F}) \\
v(\mathrm{Al}-\mathrm{C}+\mathrm{Al}-\mathrm{F}) \\
v(\mathrm{Al}-\mathrm{C})\end{array}$ & $\begin{array}{c}375 \\
613 \\
1021 \\
\end{array}$ & $\begin{array}{c}396 \\
637 \\
1051 \\
\end{array}$ & $999,1030^{[\mathrm{a}]}$ \\
\hline
\end{tabular}

[a] Raman spectra of the mixture of $[\mathrm{IPrH}][\mathrm{Ph} 3 \mathrm{AlF}](\mathbf{8})$ and $[\mathrm{IPrH}]\left[\mathrm{PhAlF}_{3}\right]$ (10) 


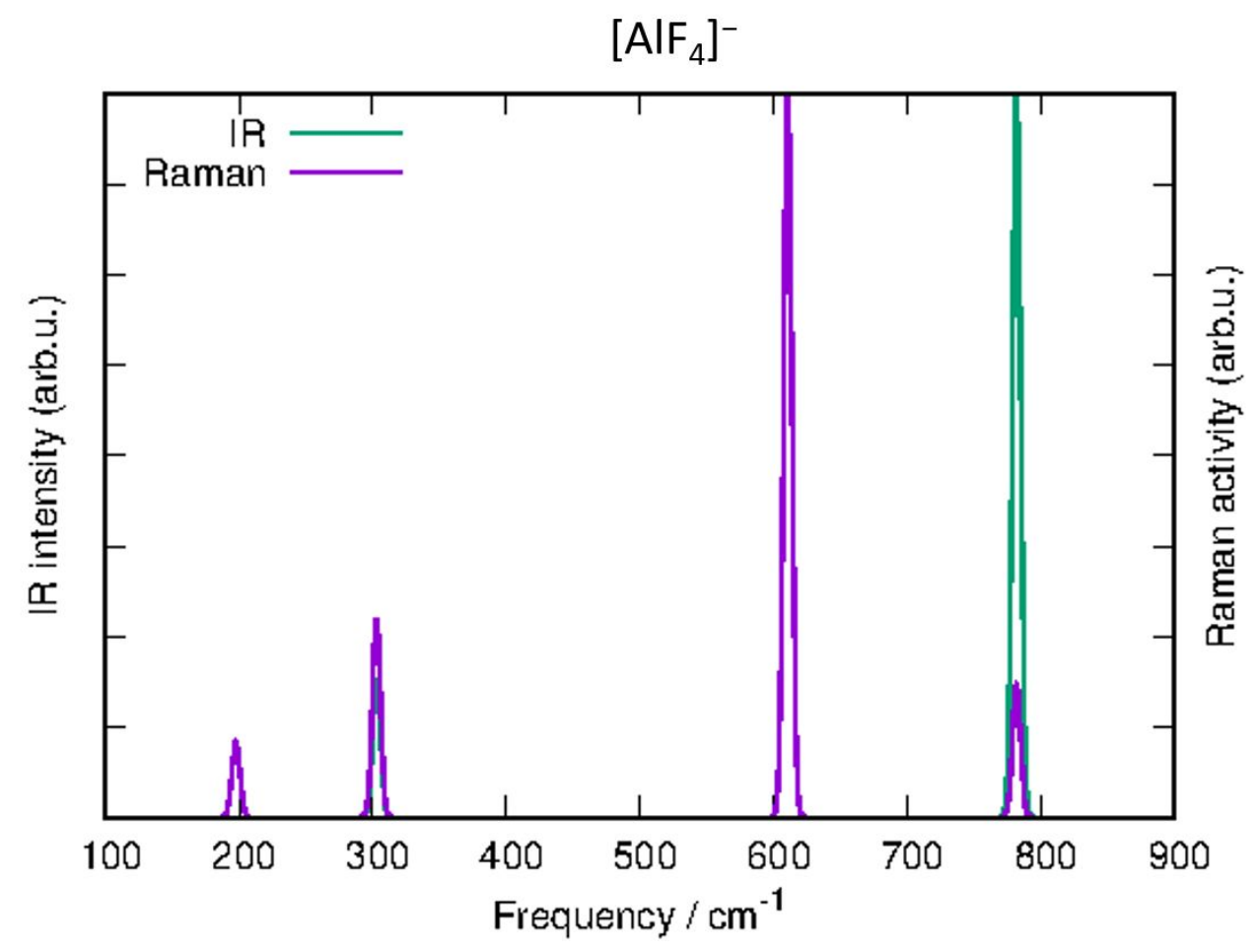

Figure S48. Calculated vibrational frequencies $(\mathrm{B} 3 \mathrm{LYP} / \mathrm{Def} 2 \mathrm{TZVP})$ for $\left[\mathrm{AlF}_{4}\right]^{-}$anion.
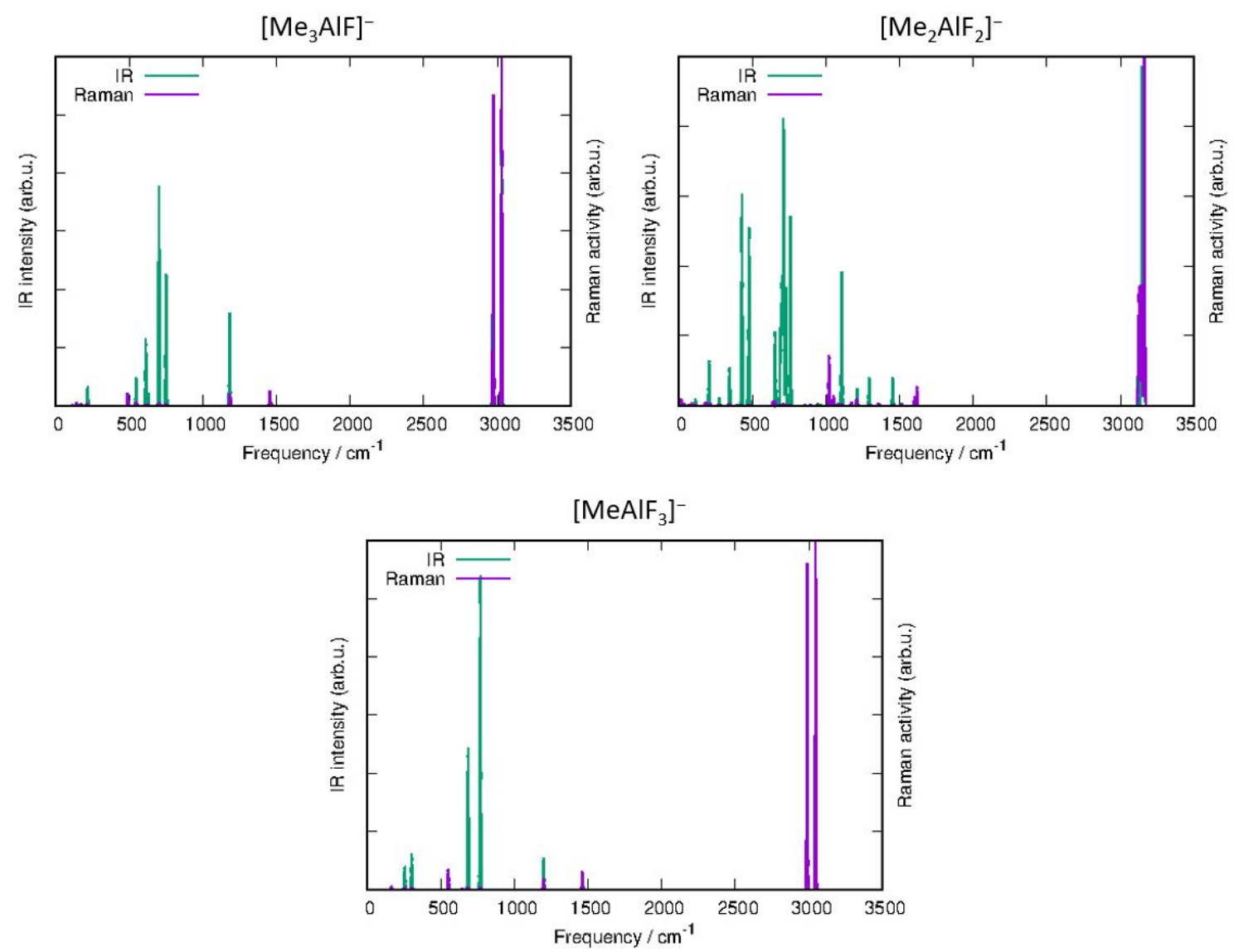

Figure S49. Calculated vibrational frequencies (B3LYP/Def2TZVP) for $\left[\mathrm{Me}_{3} \mathrm{AlF}\right]^{-}$, $\left[\mathrm{Me}_{2} \mathrm{AlF}_{2}\right]^{-}$and $\left[\mathrm{MeAlF}_{3}\right]^{-}$anions. 

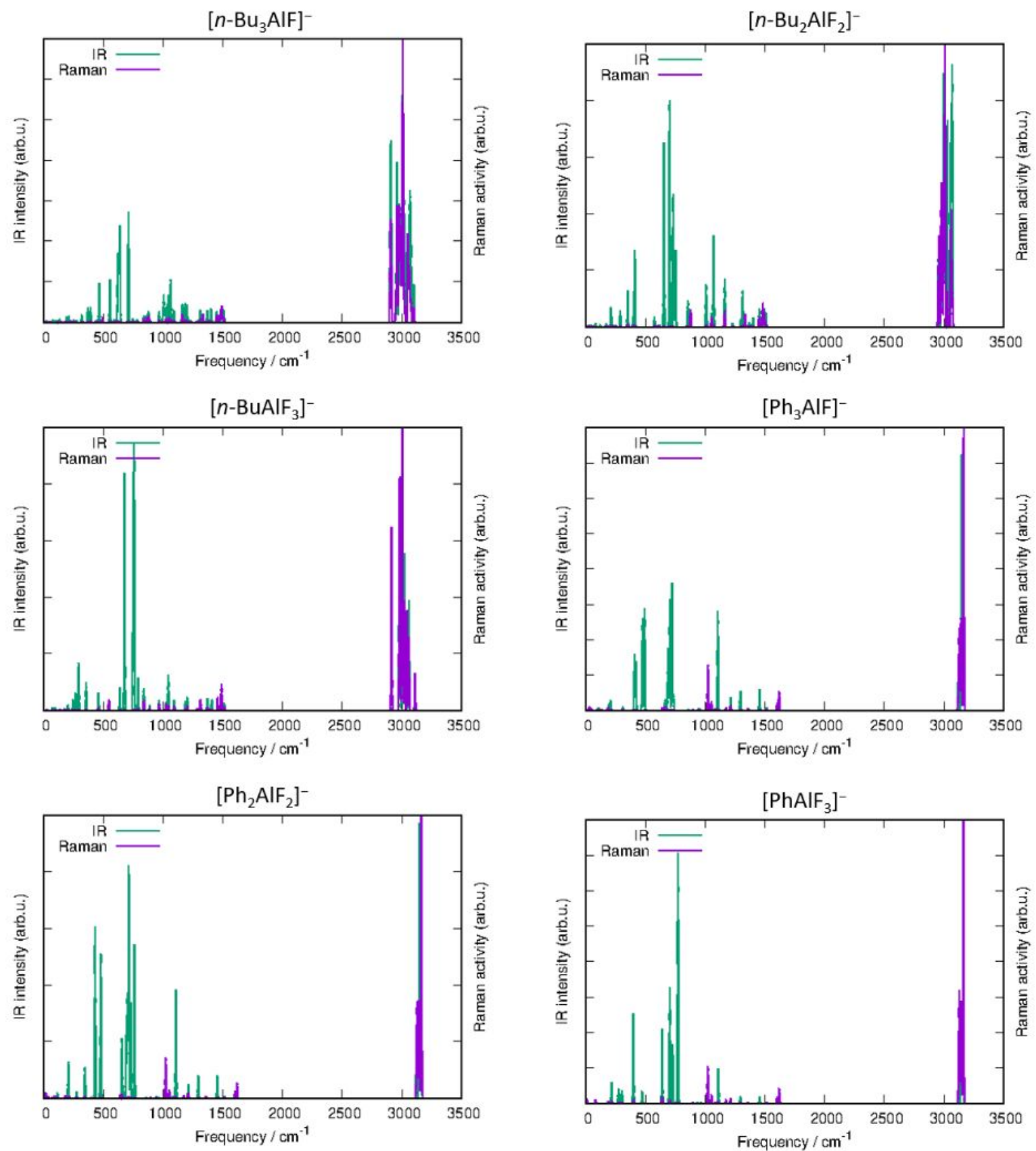

Figure S50. Calculated vibrational frequencies (B3LYP/Def2TZVP) for $\left[n-\mathrm{Bu}_{3} \mathrm{AlF}\right]^{-},[n$ $\left.\mathrm{Bu}_{2} \mathrm{AlF}_{2}\right]^{-},\left[n-\mathrm{BuAlF}_{3}\right]^{-},\left[\mathrm{Ph}_{3} \mathrm{AlF}\right]^{-},\left[\mathrm{Ph}_{2} \mathrm{AlF}_{2}\right]^{-}$and $\left[\mathrm{PhAlF}_{3}\right]^{-}$anions. 


\section{S4 Crystal Structure Data}

Table S5. Selected crystal data for $[\mathrm{IPrH}]\left[\mathrm{Me}_{2} \mathrm{AlF}_{2}\right] \cdot \mathrm{C}_{6} \mathrm{D}_{6}(\mathbf{2 a}),[\mathrm{IPrH}]\left[\mathrm{Me}_{2} \mathrm{AlF}_{2}\right] \cdot 0.95 \mathrm{DCM}$ (2b), [IPrH] $\left[\mathrm{MeAlF}_{3}\right](\mathbf{3}),[\mathrm{IPr}]\left[\mathrm{AlF}_{4}\right](\mathbf{4})$ and $[\mathrm{IPr}]\left[\mathrm{AlF}_{4}\right] \cdot \mathrm{C}_{6} \mathrm{D}_{6}(\mathbf{4 a})$.

\begin{tabular}{|c|c|c|c|c|c|}
\hline & $\begin{array}{l}{[\mathrm{IPrH}]\left[\mathrm{Me}_{2} \mathrm{AlF}_{2}\right]} \\
\cdot \mathrm{C}_{6} \mathrm{D}_{6}(\mathbf{2 a})\end{array}$ & $\begin{array}{l}{[\mathrm{IPrH}]\left[\mathrm{Me}_{2} \mathrm{AlF}_{2}\right]} \\
\cdot 0.95 \mathrm{DCM}(\mathbf{2 b})\end{array}$ & $\begin{array}{l}{[\mathrm{IPrH}]\left[\mathrm{MeAlF}_{3}\right]} \\
(\mathbf{3})\end{array}$ & {$[\mathrm{IPr}]\left[\mathrm{AlF}_{4}\right](4)$} & $\begin{array}{l}\mathrm{IPr}]\left[\mathrm{AlF}_{4}\right] \\
\cdot \mathrm{C}_{6} \mathrm{D}_{6}(\mathbf{4 a}) \\
\end{array}$ \\
\hline CCDC No. & 2116482 & 2116483 & 2116484 & 2116485 & 2116486 \\
\hline $\begin{array}{l}\text { Chemical } \\
\text { formula }\end{array}$ & $\begin{array}{l}\mathrm{C}_{27} \mathrm{H}_{37} \mathrm{~N}_{2} \cdot \\
\mathrm{AlF}_{2} \mathrm{C}_{2} \mathrm{H}_{6} . \\
\mathrm{C}_{6} \mathrm{H}_{6}\end{array}$ & $\begin{array}{l}\mathrm{C}_{27} \mathrm{H}_{37} \mathrm{~N}_{2} \cdot \\
\mathrm{AlF}_{2} \mathrm{C}_{2} \mathrm{H}_{6} . \\
\mathrm{C}_{0.95} \mathrm{H}_{1.9} \mathrm{Cl}_{1.9}\end{array}$ & $\begin{array}{l}\mathrm{C}_{27} \mathrm{H}_{37} \mathrm{~N}_{2} \\
\mathrm{AlF}_{3} \mathrm{CH}_{3}\end{array}$ & $\begin{array}{l}\mathrm{C}_{27} \mathrm{H}_{37} \mathrm{~N}_{2} \\
\mathrm{AlF}_{4}\end{array}$ & $\begin{array}{l}\mathrm{C}_{27} \mathrm{H}_{37} \mathrm{~N}_{2} \cdot \mathrm{AlF}_{4} \cdot \\
\mathrm{C}_{6} \mathrm{D}_{6}\end{array}$ \\
\hline$F_{\mathrm{W}}(\mathrm{g} / \mathrm{mol})$ & 562.74 & 565.31 & 488.60 & 492.56 & 570.67 \\
\hline$T(\mathrm{~K})$ & 150 & 150 & 150 & 150 & 150 \\
\hline$\lambda(\AA)$ & 1.54184 & 1.54184 & 1.54184 & 1.54184 & 1.54184 \\
\hline $\begin{array}{l}\text { Cristal size } \\
(\mathrm{mm})\end{array}$ & $0.88 \times 0.18 \times 0.10$ & $0.46 \times 0.28 \times 0.18$ & $0.38 \times 0.23 \times 0.12$ & $\begin{array}{l}0.35 \times 0.31 \times \\
0.22\end{array}$ & $\begin{array}{l}0.64 \times 0.05 \times \\
0.04\end{array}$ \\
\hline $\begin{array}{l}\text { Crystal } \\
\text { system }\end{array}$ & Triclinic & Triclinic & Orthorhombic & Orthorhombic & Monoclinic \\
\hline Space group & $P-1$ & $P-1$ & Pnma & $P c c n$ & $I 2 / a$ \\
\hline$a(\AA)$ & $10.0113(4)$ & $9.7859(5)$ & $16.7958(3)$ & $11.1930(2)$ & $17.202(2)$ \\
\hline$b(\AA)$ & $13.2295(5)$ & $10.7423(5)$ & $17.7836(4)$ & $12.6491(2)$ & $9.8532(6)$ \\
\hline$c(\AA)$ & $14.3963(6)$ & $16.7898(9)$ & $9.5102(2)$ & $19.9010(3)$ & $21.322(2)$ \\
\hline$\alpha\left(^{\circ}\right)$ & 113.971(4) & $81.600(4)$ & 90 & 90 & 90 \\
\hline$\beta\left(^{\circ}\right)$ & $97.920(3)$ & $85.736(4)$ & 90 & 90 & $112.44(1)$ \\
\hline$\gamma\left({ }^{\circ}\right)$ & $94.020(3)$ & $70.600(5)$ & 90 & 90 & 90 \\
\hline$V\left(\AA^{3}\right)$ & $1708.8(1)$ & $1646.3(2)$ & $2840.6(1)$ & $2817.60(8)$ & $3340.1(5)$ \\
\hline$Z$ & 2 & 2 & 4 & 4 & 4 \\
\hline$\rho_{\text {calc }}\left(\mathrm{g} / \mathrm{cm}^{3}\right)$ & 1.094 & 1.140 & 1.142 & 1.161 & 1.135 \\
\hline$\mu\left(\mathrm{mm}^{-1}\right)$ & 0.789 & 2.204 & 0.930 & 0.995 & 0.905 \\
\hline$F(000)$ & 608 & 604 & 1048 & 1048 & 1216.0 \\
\hline$\Theta$ range $\left(^{\circ}\right)$ & $3.8-73.2$ & $2.6-72.0$ & $3.6-72.2$ & $4.5-72.3$ & $4.4-71.2^{\circ}$ \\
\hline Index ranges & $\begin{aligned}-11 & \leq \mathrm{h} \leq 10 \\
-15 & \leq \mathrm{k} \leq 15 \\
-17 & \leq 1 \leq 17\end{aligned}$ & $\begin{array}{l}-12 \leq \mathrm{h} \leq 12 \\
-13 \leq \mathrm{k} \leq 13 \\
-20 \leq 1 \leq 20\end{array}$ & $\begin{aligned}-19 & \leq \mathrm{h} \leq 20 \\
-21 & \leq \mathrm{k} \leq 21 \\
-11 & \leq 1 \leq 7\end{aligned}$ & $\begin{array}{l}-8 \leq \mathrm{h} \leq 13 \\
-15 \leq \mathrm{k} \leq 15 \\
-23 \leq 1 \leq 24\end{array}$ & $\begin{array}{l}-21 \leq \mathrm{h} \leq 19 \\
-11 \leq \mathrm{k} \leq 9 \\
-24 \leq 1 \leq 26\end{array}$ \\
\hline $\begin{array}{l}\text { Reflections } \\
\text { collected }\end{array}$ & 18218 & 28031 & 19912 & 20987 & 7268 \\
\hline $\begin{array}{l}\text { Independent } \\
\text { reflections }\end{array}$ & 6054 & 6352 & 2891 & 2783 & 3215 \\
\hline $\begin{array}{l}\text { Reflections } \\
\text { with }(I> \\
2 \sigma(I)) \\
\end{array}$ & 5111 & 4773 & 2406 & 2498 & 2140 \\
\hline$R_{\text {int }}$ & 0.024 & 0.0412 & 0.0318 & 0.0232 & 0.0447 \\
\hline $\begin{array}{l}\text { Data / } \\
\text { restrains / } \\
\text { parameters }\end{array}$ & 6054 / 0 / 392 & $6352 / 0 / 381$ & $2891 / 0 / 209$ & $2783 / 0 / 159$ & $3215 / 4 / 207$ \\
\hline$S^{[\mathrm{a}]}$ & 1.035 & 1.030 & 1.044 & 1.063 & 1.020 \\
\hline $\begin{array}{l}R_{1}{ }^{[\mathrm{b}]}, w R_{2}{ }^{[\mathrm{c}]}(I \\
>2 \sigma(I))\end{array}$ & $0.0422,0.1121$ & $0.0600,0.1632$ & $0.0465,0.1234$ & $0.0431,0.1255$ & $0.0649,0.1713$ \\
\hline $\begin{array}{l}R_{1}{ }^{[\mathrm{b}]}, w R_{2}{ }^{[\mathrm{c}]} \\
\text { (all data) }\end{array}$ & $0.0513,0.1201$ & $0.0790,0.1819$ & $0.0563,0.1333$ & $0.0466,0.1300$ & $0.0982,0.1949$ \\
\hline $\begin{array}{l}\Delta \rho_{\min }, \Delta \rho_{\max } \\
\left(\mathrm{e} \AA^{-3}\right)\end{array}$ & $-0.329,0.369$ & $-0.387,0.690$ & $-0.297,0.423$ & $-0.240,0.237$ & $-0.284,0.292$ \\
\hline
\end{tabular}

[a] $S=\left[\Sigma\left(w\left(F_{\mathrm{o}}^{2}-F_{\mathrm{c}}^{2}\right)^{2}\right) /\left(N_{\mathrm{o}}-N_{\mathrm{p}}\right)\right]^{1 / 2}$.

${ }^{[b]} R_{1}=|| F_{\mathrm{o}}|-| F_{\mathrm{c}}|| \Sigma\left|F_{\mathrm{o}}\right|$.

${ }^{[c]} w R_{2}=\left[\Sigma\left(w\left(F_{\mathrm{o}}^{2}-F_{\mathrm{c}}^{2}\right)^{2}\right) / \Sigma\left(w\left(F_{\mathrm{o}}^{2}\right)^{2}\right)\right]^{1 / 2}$. 
Table S6. Selected crystal data for $[\mathrm{IPrH}]\left[n-\mathrm{Bu}_{3} \mathrm{AlF}\right](\mathbf{5}),[\mathrm{IPrH}]\left[n-\mathrm{Bu}_{2} \mathrm{AlF}_{2}\right](\mathbf{6}),[\mathrm{IPrH}][n-$ $\left.\mathrm{BuAlF}_{3}\right]$ (7), [IPrH] $\left[\mathrm{Ph}_{3} \mathrm{AlF}\right](\mathbf{8})$ and $[\mathrm{IPrH}]\left[\mathrm{PhAlF}_{3}\right](\mathbf{1 0})$.

\begin{tabular}{|c|c|c|c|c|c|}
\hline & $\begin{array}{l}{[\mathrm{IPrH}]} \\
{\left[n-\mathrm{Bu}_{3} \mathrm{AlF}\right](\mathbf{5})}\end{array}$ & $\begin{array}{l}{[\mathrm{IPrH}]} \\
{\left[n-\mathrm{Bu}_{2} \mathrm{AlF}_{2}\right](\mathbf{6})}\end{array}$ & $\begin{array}{l}{[\mathrm{IPrH}]} \\
{\left[n-\mathrm{BuAlF}_{3}\right](7)}\end{array}$ & $\begin{array}{l}{[\mathrm{IPrH}]\left[\mathrm{Ph}_{3} \mathrm{AlF}\right]} \\
(\mathbf{8})\end{array}$ & $\begin{array}{l}{[\mathrm{IPrH}]\left[\mathrm{PhAlF}_{3}\right]} \\
\mathbf{( 1 0 )}\end{array}$ \\
\hline CCDC No. & 2116487 & 2116488 & 2116489 & 2116491 & 2116490 \\
\hline $\begin{array}{l}\text { Chemical } \\
\text { formula }\end{array}$ & $\begin{array}{l}\mathrm{C}_{27} \mathrm{H}_{37} \mathrm{~N}_{2} \cdot \\
\mathrm{AlFC}_{12} \mathrm{H}_{27}\end{array}$ & $\begin{array}{l}\mathrm{C}_{27} \mathrm{H}_{37} \mathrm{~N}_{2} \cdot \\
\mathrm{AlF}_{2} \mathrm{C}_{8} \mathrm{H}_{18}\end{array}$ & $\begin{array}{l}\mathrm{C}_{27} \mathrm{H}_{37} \mathrm{~N}_{2} \cdot \\
\mathrm{AlF}_{3} \mathrm{C}_{4} \mathrm{H}_{7}\end{array}$ & $\begin{array}{l}\mathrm{C}_{27} \mathrm{H}_{37} \mathrm{~N}_{2} \cdot \\
\mathrm{AlFC}_{18} \mathrm{H}_{15}\end{array}$ & $\begin{array}{l}\mathrm{C}_{27} \mathrm{H}_{37} \mathrm{~N}_{2} \cdot \\
\mathrm{AlF}_{3} \mathrm{C}_{6} \mathrm{H}_{5}\end{array}$ \\
\hline$F_{\mathrm{W}}(\mathrm{g} / \mathrm{mol})$ & 606.90 & 568.79 & 528.66 & 666.86 & 550.66 \\
\hline$T(\mathrm{~K})$ & 189 & 150 & 240 & 150 & 150 \\
\hline$\lambda(\AA)$ & 1.54184 & 1.54184 & 1.54184 & 1.54184 & 1.54184 \\
\hline $\begin{array}{l}\text { Cristal size } \\
(\mathrm{mm})\end{array}$ & $\begin{array}{l}0.62 \times 0.28 \times \\
0.21\end{array}$ & $\begin{array}{l}0.49 \times 0.42 \times \\
0.20\end{array}$ & $\begin{array}{l}0.47 \times 0.31 \times \\
0.16\end{array}$ & $0.32 \times 0.23 \times 0.14$ & $\begin{array}{l}0.34 \times 0.27 \times \\
0.18\end{array}$ \\
\hline $\begin{array}{l}\text { Crystal } \\
\text { system }\end{array}$ & Monoclinic & Monoclinic & Monoclinic & Monoclinic & Triclinic \\
\hline Space group & $P 2_{1} / c$ & $P 2_{1} / n$ & $P 2_{1} / m$ & $P 2_{1} / n$ & $P-1$ \\
\hline$a(\AA)$ & $18.4658(4)$ & $10.8908(2)$ & $10.0812(6)$ & $17.0471(4)$ & $10.2609(3)$ \\
\hline$b(\AA)$ & $10.4398(2)$ & $19.6662(2)$ & $15.543(1)$ & $12.1991(2)$ & $10.9869(3)$ \\
\hline$c(\AA)$ & $20.1175(5)$ & 17.7021(3) & $10.9707(9)$ & $20.7609(4)$ & $14.6809(4)$ \\
\hline$\alpha\left(^{\circ}\right)$ & 90 & 90 & 90 & 90 & $90.406(3)$ \\
\hline$\beta\left(^{\circ}\right)$ & $95.789(2)$ & $107.246(2)$ & $103.460(8)$ & $112.540(2)$ & $92.012(2)$ \\
\hline$\gamma\left({ }^{\circ}\right)$ & 90 & 90 & 90 & 90 & $105.708(3)$ \\
\hline$V\left(\AA^{3}\right)$ & $3858.5(2)$ & $3621.0(1)$ & $1671.8(2)$ & $3987.6(1)$ & $1592.03(8)$ \\
\hline$Z$ & 4 & 4 & 2 & 4 & 2 \\
\hline$\rho_{\text {calc }}\left(\mathrm{g} / \mathrm{cm}^{3}\right)$ & 1.045 & 1.043 & 1.050 & 1.111 & 1.149 \\
\hline$\mu\left(\mathrm{mm}^{-1}\right)$ & 0.685 & 0.745 & 0.823 & 0.717 & 0.886 \\
\hline$F(000)$ & 1336 & 1240 & 568 & 1432 & 588 \\
\hline$\Theta$ range $\left({ }^{\circ}\right)$ & $3.4-72.3$ & $3.4-72.3$ & $4.1-72.2$ & $4.2-72.3$ & $3.0-72.2$ \\
\hline $\begin{array}{l}\text { Index } \\
\text { ranges }\end{array}$ & $\begin{array}{l}-22 \leq \mathrm{h} \leq 22 \\
-12 \leq \mathrm{k} \leq 12 \\
-17 \leq 1 \leq 23\end{array}$ & $\begin{array}{l}-13 \leq \mathrm{h} \leq 13 \\
-16 \leq \mathrm{k} \leq 26 \\
-21 \leq 1 \leq 20\end{array}$ & $\begin{array}{l}-10 \leq \mathrm{h} \leq 12 \\
-18 \leq \mathrm{k} \leq 18 \\
-11 \leq 1 \leq 13\end{array}$ & $\begin{array}{l}-21 \leq \mathrm{h} \leq 20 \\
-10 \leq \mathrm{k} \leq 14 \\
-23 \leq 1 \leq 25\end{array}$ & $\begin{array}{l}-12 \leq \mathrm{h} \leq 12 \\
-13 \leq \mathrm{k} \leq 13 \\
-17 \leq 1 \leq 17\end{array}$ \\
\hline $\begin{array}{l}\text { Reflections } \\
\text { collected }\end{array}$ & 27379 & 25803 & 7359 & 40111 & 48472 \\
\hline $\begin{array}{l}\text { Independent } \\
\text { reflections }\end{array}$ & 7400 & 7080 & 3346 & 7826 & 5687 \\
\hline $\begin{array}{l}\text { Reflections } \\
\text { with }(I> \\
2 \sigma(I)) \\
\end{array}$ & 5692 & 6308 & 2054 & 6596 & 4327 \\
\hline$R_{\text {int }}$ & 0.0297 & 0.0183 & 0.0257 & 0.0305 & 0.0575 \\
\hline $\begin{array}{l}\text { Data / } \\
\text { restrains / } \\
\text { parameters }\end{array}$ & $7400 / 0 / 477$ & $7080 / 0 / 441$ & $3346 / 0 / 188$ & $7826 / 0 / 450$ & $5687 / 0 / 379$ \\
\hline$S^{[\mathrm{a}]}$ & 1.049 & 1.017 & 1.060 & 1.033 & 1.037 \\
\hline $\begin{array}{l}R_{1}{ }^{[\mathrm{b}]}, w R_{2}{ }^{[\mathrm{c}]} \\
(I>2 \sigma(I))\end{array}$ & $0.0574,0.1571$ & $0.0462,0.1287$ & $0.0828,0.2484$ & $0.0454,0.1255$ & $0.0694,0.1980$ \\
\hline $\begin{array}{l}R_{1}{ }^{[\mathrm{b}]}, w R_{2}{ }^{[\mathrm{c}]} \\
\text { (all data) }\end{array}$ & $0.0743,0.1759$ & $0.0512,0.1344$ & $0.1153,0.2905$ & $0.0538,0.1338$ & $0.0876,0.2148$ \\
\hline $\begin{array}{l}\Delta \rho_{\min }, \Delta \rho_{\max } \\
\left(\mathrm{e} \AA^{-3}\right)\end{array}$ & $-0.238,0.530$ & $-0.341,0.353$ & $-0.332,0.465$ & $-0.291,0.377$ & $-0.316,0.595$ \\
\hline
\end{tabular}


Table S7. Selected bond lengths $(\AA)$ and bond angles $\left({ }^{\circ}\right)$ for $[\mathrm{IPrH}]\left[\mathrm{Me}_{2} \mathrm{AlF}_{2}\right] \cdot \mathrm{C}_{6} \mathrm{D}_{6}(\mathbf{2 a})$, $[\mathrm{IPrH}]\left[\mathrm{Me}_{2} \mathrm{AlF}_{2}\right] \cdot 0.95 \mathrm{DCM}(\mathbf{2 b}),[\mathrm{IPrH}]\left[\mathrm{MeAlF}_{3}\right](\mathbf{3}),[\mathrm{IPr}]\left[\mathrm{AlF}_{4}\right](\mathbf{4}),[\mathrm{IPr}]\left[\mathrm{AlF}_{4}\right] \cdot \mathrm{C}_{6} \mathrm{D}_{6}(\mathbf{4 a})$, $[\mathrm{IPrH}]\left[n-\mathrm{Bu}_{3} \mathrm{AlF}\right](\mathbf{5}),[\mathrm{IPrH}]\left[n-\mathrm{Bu}_{2} \mathrm{AlF}_{2}\right](\mathbf{6}),[\mathrm{IPrH}]\left[n-\mathrm{BuAlF}{ }_{3}\right](7),[\mathrm{IPrH}]\left[\mathrm{Ph}_{3} \mathrm{AlF}\right](\mathbf{8})$ and $[\mathrm{IPrH}]\left[\mathrm{PhAlF}_{3}\right](\mathbf{1 0})$.

\begin{tabular}{|c|c|c|c|}
\hline \multicolumn{4}{|c|}{$[\mathrm{IPrH}]\left[\mathrm{Me}_{2} \mathrm{AlF}_{2}\right] \cdot \mathrm{C}_{6} \mathrm{D}_{6}(\mathbf{2 a})$} \\
\hline \multicolumn{2}{|c|}{ Bond lengths } & \multicolumn{2}{|l|}{ Bond angles } \\
\hline $\mathrm{Al}(1)-\mathrm{F}(1)$ & $1.711(1)$ & $\mathrm{F}(1)-\mathrm{Al}(1)-\mathrm{F}(2)$ & $103.66(6)$ \\
\hline $\mathrm{Al}(1)-\mathrm{F}(2)$ & $1.717(1)$ & $\mathrm{F}(1)-\mathrm{Al}(1)-\mathrm{C}(1)$ & $109.58(7)$ \\
\hline $\mathrm{Al}(1)-\mathrm{C}(1)$ & $1.951(2)$ & $\mathrm{F}(1)-\mathrm{Al}(1)-\mathrm{C}(2)$ & $108.63(7)$ \\
\hline \multirow{2}{*}{$\mathrm{Al}(1)-\mathrm{C}(2)$} & $1.965(2)$ & $\mathrm{F}(2)-\mathrm{Al}(1)-\mathrm{C}(1)$ & $109.47(7)$ \\
\hline & & $\mathrm{F}(2)-\mathrm{Al}(1)-\mathrm{C}(2)$ & $108.93(8)$ \\
\hline \multicolumn{4}{|c|}{$[\mathrm{IPrH}]\left[\mathrm{Me}_{2} \mathrm{AlF}_{2}\right] \cdot 0.95 \mathrm{DCM}(\mathbf{2 b})$} \\
\hline \multicolumn{2}{|c|}{ Bond lengths } & \multicolumn{2}{|l|}{ Bond angles } \\
\hline $\mathrm{Al}(1)-\mathrm{F}(1 \mathrm{~A})$ & $1.601(3)$ & $\mathrm{F}(1 \mathrm{~A})-\mathrm{Al}(1)-\mathrm{F}(2 \mathrm{~A})$ & $104.6(3)$ \\
\hline $\mathrm{Al}(1)-\mathrm{F}(1 \mathrm{~B})$ & $1.923(4)$ & $\mathrm{F}(1 \mathrm{~B})-\mathrm{Al}(1)-\mathrm{F}(2 \mathrm{~B})$ & 104.1(2) \\
\hline $\mathrm{Al}(1)-\mathrm{F}(2 \mathrm{~A})$ & $1.878(4)$ & $\mathrm{F}(1 \mathrm{~A})-\mathrm{Al}(1)-\mathrm{C}(1)$ & $111.1(2)$ \\
\hline $\mathrm{Al}(1)-\mathrm{F}(2 \mathrm{~B})$ & $1.902(5)$ & $\mathrm{F}(1 \mathrm{~A})-\mathrm{Al}(1)-\mathrm{C}(2)$ & $115.6(2)$ \\
\hline $\mathrm{Al}(1)-\mathrm{C}(1)$ & $1.955(3)$ & $\mathrm{F}(1 \mathrm{~B})-\mathrm{Al}(1)-\mathrm{C}(1)$ & $101.4(2)$ \\
\hline \multirow{5}{*}{$\mathrm{Al}(1)-\mathrm{C}(2)$} & $1.977(3)$ & $\mathrm{F}(1 \mathrm{~B})-\mathrm{Al}(1)-\mathrm{C}(2)$ & $98.4(2)$ \\
\hline & & $\mathrm{F}(2 \mathrm{~A})-\mathrm{Al}(1)-\mathrm{C}(1)$ & $98.5(2)$ \\
\hline & & $\mathrm{F}(2 \mathrm{~A})-\mathrm{Al}(1)-\mathrm{C}(2)$ & $107.7(1)$ \\
\hline & & $\mathrm{F}(2 \mathrm{~B})-\mathrm{Al}(1)-\mathrm{C}(1)$ & $121.6(2)$ \\
\hline & & $\mathrm{F}(2 \mathrm{~B})-\mathrm{Al}(1)-\mathrm{C}(2)$ & $109.9(2)$ \\
\hline \multicolumn{4}{|c|}{$[\mathrm{IPrH}]\left[\mathrm{MeAlF}_{3}\right](\mathbf{3})$} \\
\hline \multicolumn{2}{|c|}{ Bond lengths } & \multicolumn{2}{|l|}{ Bond angles } \\
\hline $\mathrm{Al}(1)-\mathrm{F}(1)$ & $1.683(1)$ & $\mathrm{F}(1)-\mathrm{Al}(1)-\mathrm{F}\left(1^{\mathrm{I}}\right)$ & $105.02(9)$ \\
\hline $\mathrm{Al}(1)-\mathrm{F}\left(1^{\mathrm{I}}\right)$ & $1.683(1)$ & $\mathrm{F}(1)-\mathrm{Al}(1)-\mathrm{F}(2)$ & $100.6(6)$ \\
\hline $\mathrm{Al}(1)-\mathrm{F}(2)$ & $1.696(3)$ & $\mathrm{F}\left(1^{1}\right)-\mathrm{Al}(1)-\mathrm{F}(2)$ & $113.0(6)$ \\
\hline \multirow[t]{3}{*}{$\mathrm{Al}(1)-\mathrm{C}(1)$} & $1.945(3)$ & $\mathrm{F}(1)-\mathrm{Al}(1)-\mathrm{C}(1)$ & $114.33(7)$ \\
\hline & & $\mathrm{F}\left(1^{\mathrm{I}}\right)-\mathrm{Al}(1)-\mathrm{C}(1)$ & $114.33(8)$ \\
\hline & & $\mathrm{F}(2)-\mathrm{Al}(1)-\mathrm{C}(1)$ & $108.8(2)$ \\
\hline \multicolumn{4}{|c|}{$[\mathrm{IPr}]\left[\mathrm{AlF}_{4}\right](4)$} \\
\hline \multicolumn{2}{|c|}{ Bond lengths } & \multicolumn{2}{|l|}{ Bond angles } \\
\hline $\mathrm{Al}(1)-\mathrm{F}(1)$ & $1.660(1)$ & $\mathrm{F}(1)-\mathrm{Al}(1)-\mathrm{F}\left(1^{\mathrm{I}}\right)$ & $105.80(9)$ \\
\hline $\mathrm{Al}(1)-\mathrm{F}\left(1^{\mathrm{I}}\right)$ & $1.660(1)$ & $\mathrm{F}(1)-\mathrm{Al}(1)-\mathrm{F}(2)$ & $111.27(6)$ \\
\hline $\mathrm{Al}(1)-\mathrm{F}(2)$ & $1.669(1)$ & $\mathrm{F}\left(1^{1}\right)-\mathrm{Al}(1)-\mathrm{F}(2)$ & $109.75(5)$ \\
\hline \multirow[t]{3}{*}{$\mathrm{Al}(1)-\mathrm{F}\left(2^{\mathrm{I}}\right)$} & $1.669(1)$ & $\mathrm{F}(1)-\mathrm{Al}(1)-\mathrm{F}\left(2^{\mathrm{I}}\right)$ & $109.75(5)$ \\
\hline & & $\mathrm{F}\left(1^{\mathrm{I}}\right)-\mathrm{Al}(1)-\mathrm{F}\left(2^{\mathrm{I}}\right)$ & $111.27(6)$ \\
\hline & & $\mathrm{F}(2)-\mathrm{Al}(1)-\mathrm{F}\left(2^{\mathrm{I}}\right)$ & $109.00(8)$ \\
\hline \multicolumn{4}{|c|}{$[\mathrm{IPr}]\left[\mathrm{AlF}_{4}\right] \cdot \mathrm{C}_{6} \mathrm{D}_{6}(\mathbf{4 a})$} \\
\hline \multicolumn{2}{|c|}{ Bond lengths } & \multicolumn{2}{|l|}{ Bond angles } \\
\hline $\mathrm{Al}(1)-\mathrm{F}(1)$ & $1.660(2)$ & $\mathrm{F}(1)-\mathrm{Al}(1)-\mathrm{F}\left(1^{\mathrm{I}}\right)$ & $105.8(2)$ \\
\hline $\mathrm{Al}(1)-\mathrm{F}\left(1^{\mathrm{I}}\right)$ & $1.660(2)$ & $\mathrm{F}(1)-\mathrm{Al}(1)-\mathrm{F}(2)$ & $110.9(1)$ \\
\hline $\mathrm{Al}(1)-\mathrm{F}(2)$ & $1.672(3)$ & $\mathrm{F}\left(1^{\mathrm{I}}\right)-\mathrm{Al}(1)-\mathrm{F}(2)$ & $110.3(1)$ \\
\hline \multirow[t]{3}{*}{$\mathrm{Al}(1)-\mathrm{F}\left(2^{\mathrm{I}}\right)$} & $1.672(3)$ & $\mathrm{F}(1)-\mathrm{Al}(1)-\mathrm{F}\left(2^{\mathrm{I}}\right)$ & $110.3(1)$ \\
\hline & & $\mathrm{F}\left(1^{\mathrm{I}}\right)-\mathrm{Al}(1)-\mathrm{F}\left(2^{\mathrm{I}}\right)$ & $110.9(1)$ \\
\hline & & $\mathrm{F}(2)-\mathrm{Al}(1)-\mathrm{F}\left(2^{\mathrm{I}}\right)$ & $108.5(2)$ \\
\hline
\end{tabular}




\begin{tabular}{|c|c|c|c|}
\hline \multicolumn{4}{|c|}{$[\mathrm{IPrH}]\left[n-\mathrm{Bu}_{3} \mathrm{AlF}\right](\mathbf{5})$} \\
\hline \multicolumn{2}{|c|}{ Bond lengths } & \multicolumn{2}{|l|}{ Bond angles } \\
\hline $\mathrm{Al}(1)-\mathrm{F}(1)$ & $1.733(1)$ & $\mathrm{F}(1)-\mathrm{Al}(1)-\mathrm{C}(1)$ & $109.0(1)$ \\
\hline $\mathrm{Al}(1)-\mathrm{C}(1)$ & $1.984(3)$ & $\mathrm{F}(1)-\mathrm{Al}(1)-\mathrm{C}(5)$ & 106.2(1) \\
\hline $\mathrm{Al}(1)-\mathrm{C}(5)$ & $1.994(3)$ & $\mathrm{F}(1)-\mathrm{Al}(1)-\mathrm{C}(9)$ & $106.4(1)$ \\
\hline \multirow[t]{3}{*}{$\mathrm{Al}(1)-\mathrm{C}(9)$} & $2.013(3)$ & $\mathrm{C}(1)-\mathrm{Al}(1)-\mathrm{C}(5)$ & $113.4(2)$ \\
\hline & & $\mathrm{C}(1)-\mathrm{Al}(1)-\mathrm{C}(9)$ & $115.0(1)$ \\
\hline & & $\mathrm{C}(5)-\mathrm{Al}(1)-\mathrm{C}(9)$ & $106.3(2)$ \\
\hline \multicolumn{4}{|c|}{$[\mathrm{IPrH}]\left[n-\mathrm{Bu}_{2} \mathrm{AlF}_{2}\right](\mathbf{6})$} \\
\hline Bond lengths & & Bond angles & \\
\hline $\mathrm{Al}(1)-\mathrm{F}(1)$ & $1.7072(9)$ & $\mathrm{F}(1)-\mathrm{Al}(1)-\mathrm{F}(2)$ & $103.50(5)$ \\
\hline $\mathrm{Al}(1)-\mathrm{F}(2)$ & $1.7096(9)$ & $\mathrm{F}(1)-\mathrm{Al}(1)-\mathrm{C}(1)$ & $106.49(6)$ \\
\hline $\mathrm{Al}(1)-\mathrm{C}(1)$ & $1.980(2)$ & $\mathrm{F}(1)-\mathrm{Al}(1)-\mathrm{C}(5 \mathrm{~A})$ & $110.4(1)$ \\
\hline $\mathrm{Al}(1)-\mathrm{C}(5 \mathrm{~A})$ & $2.073(4)$ & $\mathrm{F}(1)-\mathrm{Al}(1)-\mathrm{C}(5 \mathrm{~B})$ & $103.8(2)$ \\
\hline \multirow[t]{3}{*}{$\mathrm{Al}(1)-\mathrm{C}(5 \mathrm{~B})$} & $1.872(6)$ & $\mathrm{F}(2)-\mathrm{Al}(1)-\mathrm{C}(1)$ & $108.94(6)$ \\
\hline & & $\mathrm{F}(2)-\mathrm{Al}(1)-\mathrm{C}(5 \mathrm{~A})$ & $103.7(2)$ \\
\hline & & $\mathrm{F}(2)-\mathrm{Al}(1)-\mathrm{C}(5 \mathrm{~B})$ & $119.9(2)$ \\
\hline \multicolumn{4}{|c|}{$[\mathrm{IPrH}]\left[n-\mathrm{BuAlF}_{3}\right](7)$} \\
\hline \multicolumn{2}{|c|}{ Bond lengths } & \multicolumn{2}{|l|}{ Bond angles } \\
\hline $\mathrm{Al}(1)-\mathrm{F}(1 \mathrm{~A})$ & $1.539(4)$ & $\mathrm{F}(1 \mathrm{~A})-\mathrm{Al}(1)-\mathrm{F}(2 \mathrm{~A})$ & $117.4(3)$ \\
\hline $\mathrm{Al}(1)-\mathrm{F}(1 \mathrm{~B})$ & $1.64(2)$ & $\mathrm{F}(1 \mathrm{~A})-\mathrm{Al}(1)-\mathrm{F}\left(2 \mathrm{~A}^{\mathrm{I}}\right)$ & $117.4(3)$ \\
\hline $\mathrm{Al}(1)-\mathrm{F}\left(1 \mathrm{~B}^{\mathrm{I}}\right)$ & $1.64(2)$ & $\mathrm{F}(2 \mathrm{~A})-\mathrm{Al}(1)-\mathrm{F}\left(2 \mathrm{~A}^{\mathrm{I}}\right)$ & $113.8(6)$ \\
\hline $\mathrm{Al}(1)-\mathrm{F}(2 \mathrm{~A})$ & $1.592(5)$ & $\mathrm{F}(1 \mathrm{~B})-\mathrm{Al}(1)-\mathrm{F}\left(1 \mathrm{~B}^{\mathrm{I}}\right)$ & $79(3)$ \\
\hline $\mathrm{Al}(1)-\mathrm{F}\left(2 \mathrm{~A}^{\mathrm{I}}\right)$ & $1.592(5)$ & $\mathrm{F}(1 \mathrm{~B})-\mathrm{Al}(1)-\mathrm{F}(2 \mathrm{~B})$ & $93(1)$ \\
\hline $\mathrm{Al}(1)-\mathrm{F}(2 \mathrm{~B})$ & $1.66(1)$ & $\mathrm{F}(1 \mathrm{~B})-\mathrm{Al}(1)-\mathrm{F}\left(2 \mathrm{~B}^{\mathrm{I}}\right)$ & $93(1)$ \\
\hline \multirow[t]{3}{*}{$\mathrm{Al}(1)-\mathrm{C}(1)$} & $1.92(2)$ & $\mathrm{F}(1 \mathrm{~A})-\mathrm{Al}(1)-\mathrm{C}(1)$ & $108(1)$ \\
\hline & & $\mathrm{F}(2 \mathrm{~A})-\mathrm{Al}(1)-\mathrm{C}(1)$ & $114.8(8)$ \\
\hline & & $\mathrm{F}\left(2 \mathrm{~A}^{\mathrm{I}}\right)-\mathrm{Al}(1)-\mathrm{C}(1)$ & $79.6(8)$ \\
\hline \multicolumn{4}{|c|}{$[\mathrm{IPrH}]\left[\mathrm{Ph}_{3} \mathrm{AlF}\right](\mathbf{8})$} \\
\hline \multicolumn{2}{|c|}{ Bond lengths } & \multicolumn{2}{|l|}{ Bond angles } \\
\hline $\mathrm{Al}(1)-\mathrm{F}(1)$ & $1.730(1)$ & $\mathrm{F}(1)-\mathrm{Al}(1)-\mathrm{C}(1)$ & $111.31(6)$ \\
\hline $\mathrm{Al}(1)-\mathrm{C}(1)$ & $2.000(2)$ & $\mathrm{F}(1)-\mathrm{Al}(1)-\mathrm{C}(7)$ & $103.75(6)$ \\
\hline $\mathrm{Al}(1)-\mathrm{C}(7)$ & $2.004(2)$ & $\mathrm{F}(1)-\mathrm{Al}(1)-\mathrm{C}(13)$ & $104.93(6)$ \\
\hline \multirow[t]{3}{*}{$\mathrm{Al}(1)-\mathrm{C}(13)$} & $2.004(2)$ & $\mathrm{C}(1)-\mathrm{Al}(1)-\mathrm{C}(7)$ & $110.54(7)$ \\
\hline & & $\mathrm{C}(1)-\mathrm{Al}(1)-\mathrm{C}(13)$ & $111.60(7)$ \\
\hline & & $\mathrm{C}(7)-\mathrm{Al}(1)-\mathrm{C}(13)$ & $114.29(6)$ \\
\hline \multicolumn{4}{|c|}{$[\mathrm{IPrH}]\left[\mathrm{PhAlF}_{3}\right](\mathbf{1 0})$} \\
\hline \multicolumn{2}{|c|}{ Bond lengths } & \multicolumn{2}{|l|}{ Bond angles } \\
\hline $\mathrm{Al}(1)-\mathrm{F}(1)$ & $1.683(2)$ & $\mathrm{F}(1)-\mathrm{Al}(1)-\mathrm{F}(2 \mathrm{~A})$ & $104.3(1)$ \\
\hline $\mathrm{Al}(1)-\mathrm{F}(2 \mathrm{~A})$ & $2.155(4)$ & $\mathrm{F}(1)-\mathrm{Al}(1)-\mathrm{F}(2 \mathrm{~B})$ & $113.7(4)$ \\
\hline $\mathrm{Al}(1)-\mathrm{F}(2 \mathrm{~B})$ & $1.698(9)$ & $\mathrm{F}(1)-\mathrm{Al}(1)-\mathrm{F}(3 \mathrm{~A})$ & $109.2(2)$ \\
\hline $\mathrm{Al}(1)-\mathrm{F}(3 \mathrm{~A})$ & $1.703(6)$ & $\mathrm{F}(1)-\mathrm{Al}(1)-\mathrm{F}(3 \mathrm{~B})$ & 104.3(3) \\
\hline $\mathrm{Al}(1)-\mathrm{F}(3 \mathrm{~B})$ & $1.944(9)$ & $\mathrm{F}(2 \mathrm{~A})-\mathrm{Al}(1)-\mathrm{F}(3 \mathrm{~A})$ & $106.9(3)$ \\
\hline \multirow[t]{5}{*}{$\mathrm{Al}(1)-\mathrm{C}(1)$} & $1.960(3)$ & $\mathrm{F}(2 \mathrm{~B})-\mathrm{Al}(1)-\mathrm{F}(3 \mathrm{~B})$ & $104.0(5)$ \\
\hline & & $\mathrm{F}(2 \mathrm{~A})-\mathrm{Al}(1)-\mathrm{C}(1)$ & $106.7(1)$ \\
\hline & & $\mathrm{F}(2 \mathrm{~B})-\mathrm{Al}(1)-\mathrm{C}(1)$ & $115.4(2)$ \\
\hline & & $\mathrm{F}(3 \mathrm{~A})-\mathrm{Al}(1)-\mathrm{C}(1)$ & $116.8(3)$ \\
\hline & & $\mathrm{F}(3 \mathrm{~B})-\mathrm{Al}(1)-\mathrm{C}(1)$ & $106.2(3)$ \\
\hline
\end{tabular}



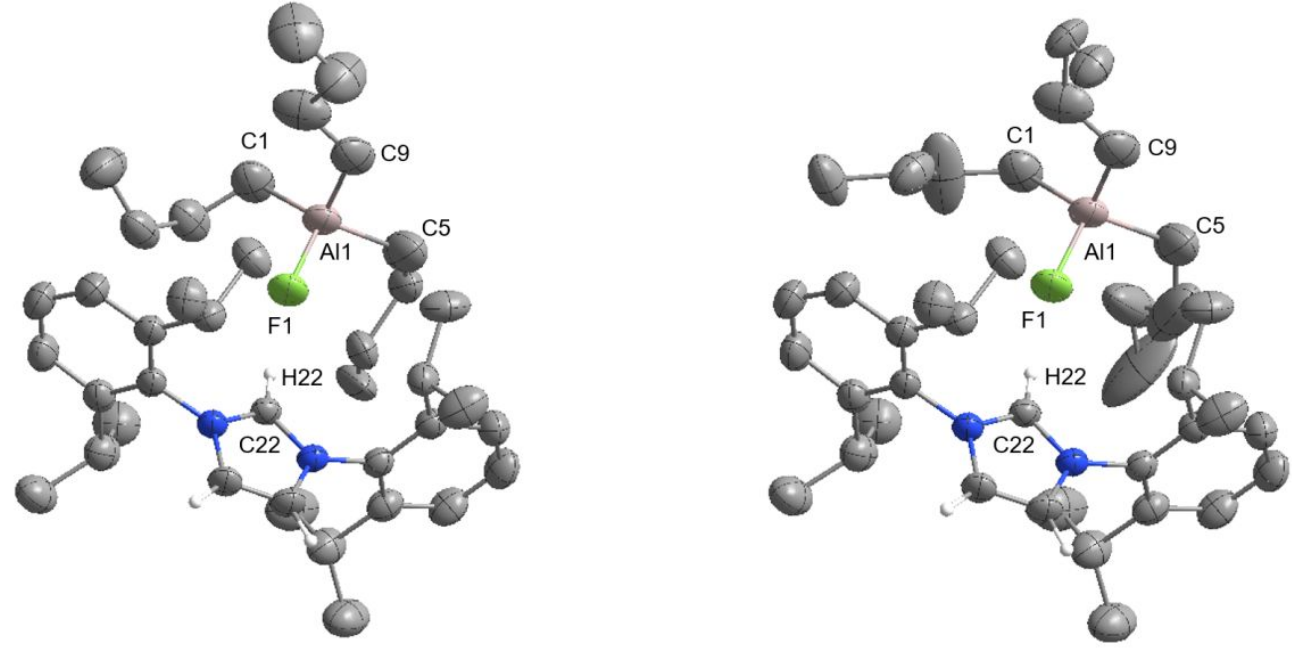

Figure S51. Crystal structure of the asymmetric unit of domain A (left) and domain B (right) of $[\mathrm{IPrH}]\left[n-\mathrm{Bu}_{3} \mathrm{AlF}\right](\mathbf{5})$. The ellipsoids are drawn at $50 \%$ probability. For clarity, all hydrogen atoms are omitted except those on the imidazolium ring.

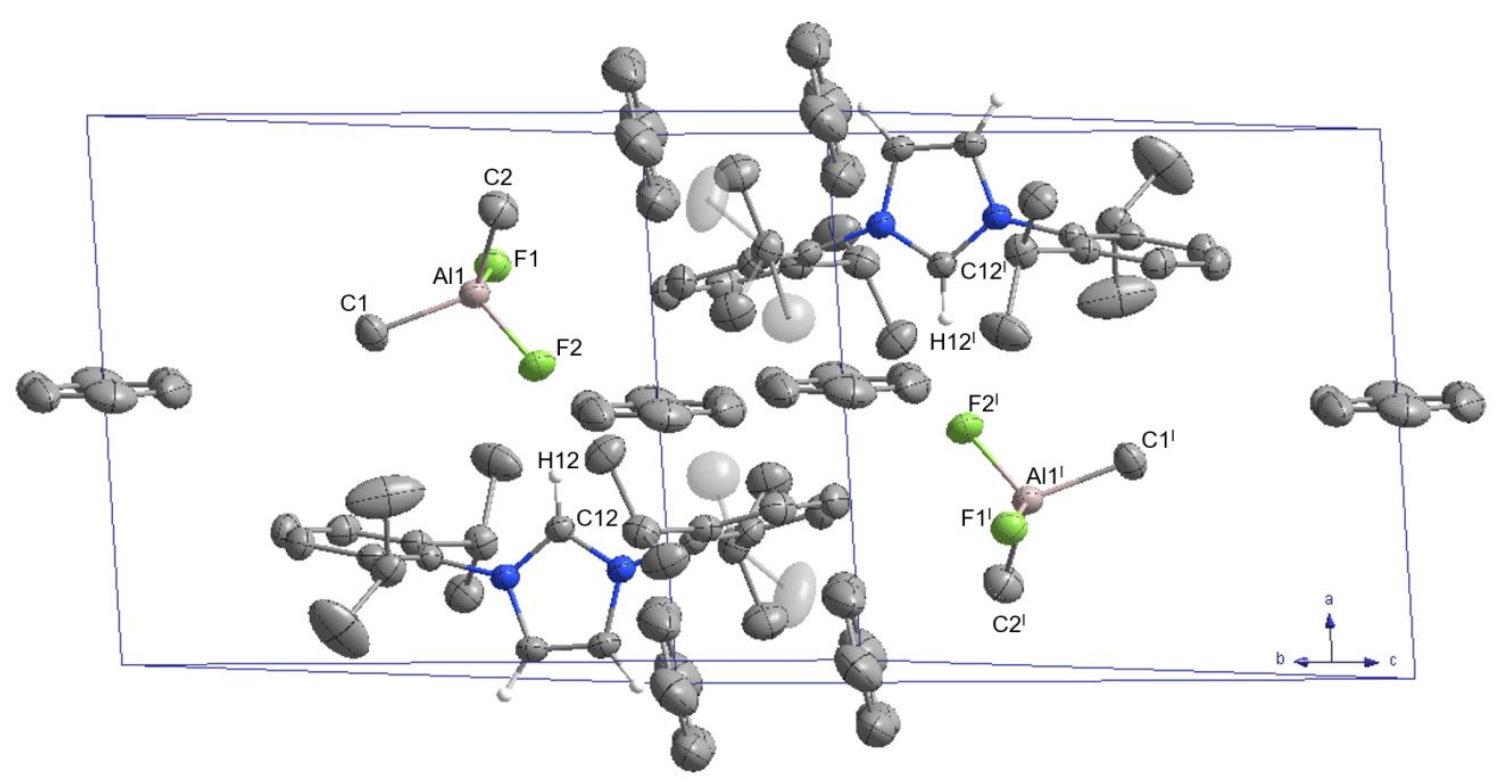

Figure S52. Unit cell packing in the crystal structure of $[\operatorname{IPrH}]\left[\mathrm{Me}_{2} \mathrm{AlF}_{2}\right] \cdot \mathrm{C}_{6} \mathrm{D}_{6}(\mathbf{2 a})$. The ellipsoids are drawn at $50 \%$ probability. The positions of disordered atoms are shown in domain A and B. For clarity, domain B is shaded and all hydrogen atoms are omitted, except those on the imidazolium ring. 

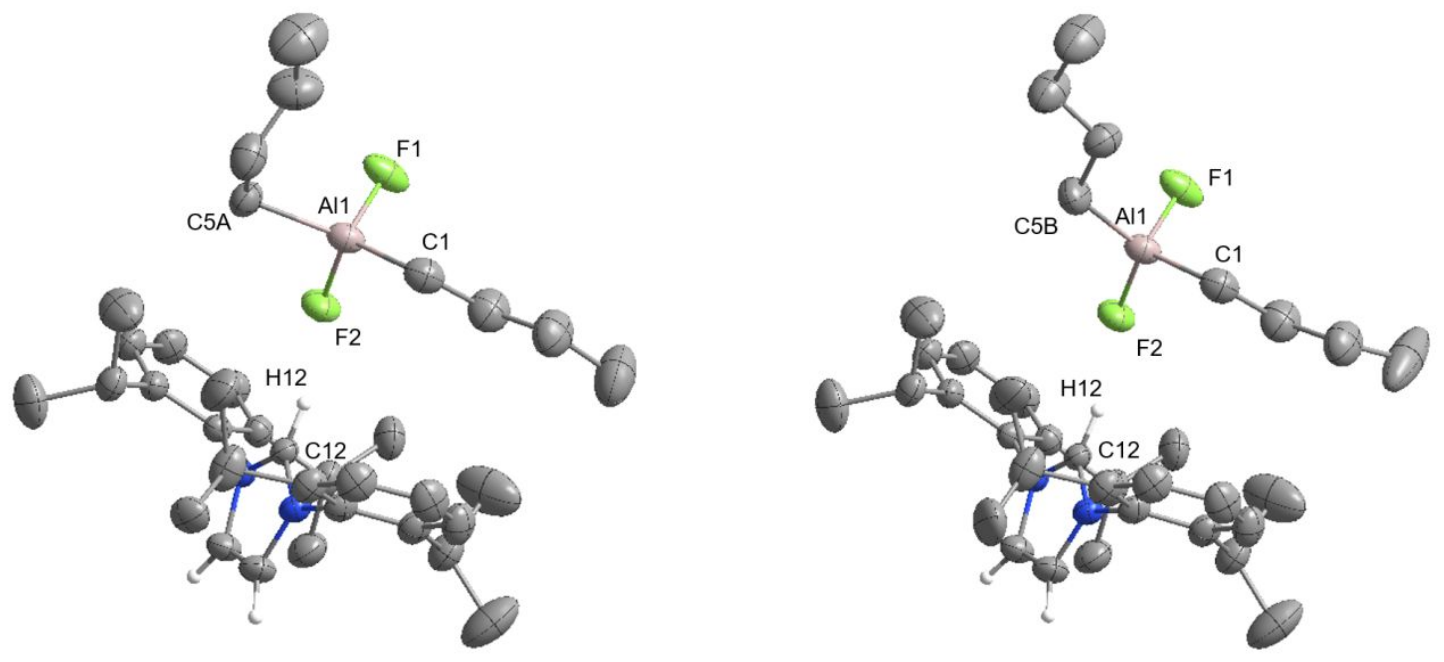

Figure S53. Crystal structure of the asymmetric unit of domain A (left) and domain B (right) of $[\mathrm{IPrH}]\left[n-\mathrm{Bu}_{2} \mathrm{AlF}_{2}\right](6)$. The ellipsoids are drawn at $50 \%$ probability. For clarity, all hydrogen atoms are omitted, except those on the imidazolium ring.
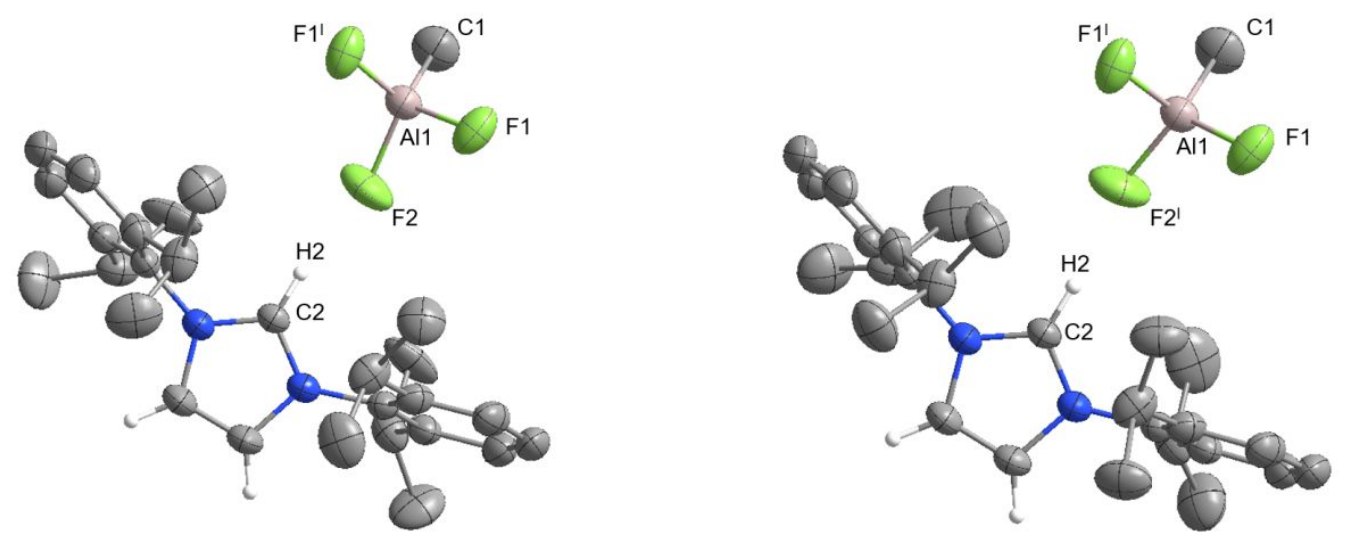

Figure S54. Crystal structure of domain A (left) and domain B (right) of [IPrH] $\left[\mathrm{MeAlF} \mathrm{F}_{3}\right](\mathbf{3})$. The ellipsoids are drawn at 50\% probability. For clarity, all hydrogen atoms are omitted except those on the imidazolium ring. Symmetry code: (i) $x, 1 / 2-y, z$. 

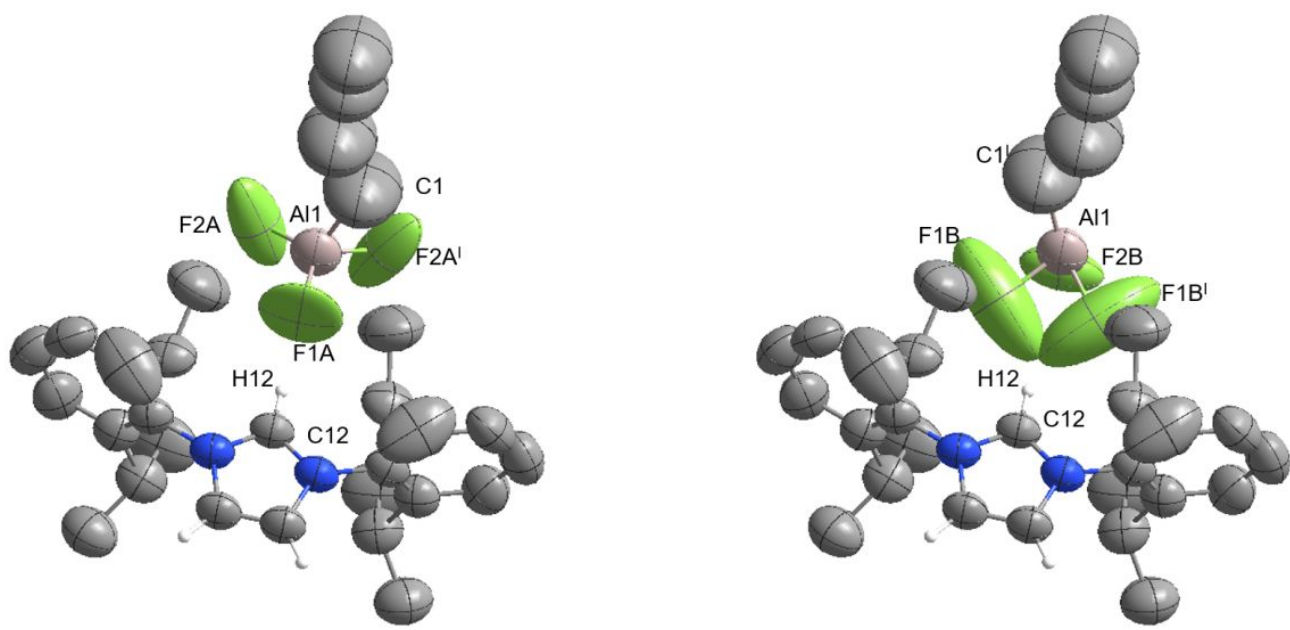

Figure S55. Crystal structure of domain A (left) and domain B (right) of [IPrH] $\left[n-\mathrm{BuAlF}_{3}\right]$ (7). The ellipsoids are drawn at 50\% probability. For clarity, all hydrogen atoms are omitted except those on the imidazolium ring. Symmetry code: (i) $x, 1 / 2-y, z$.
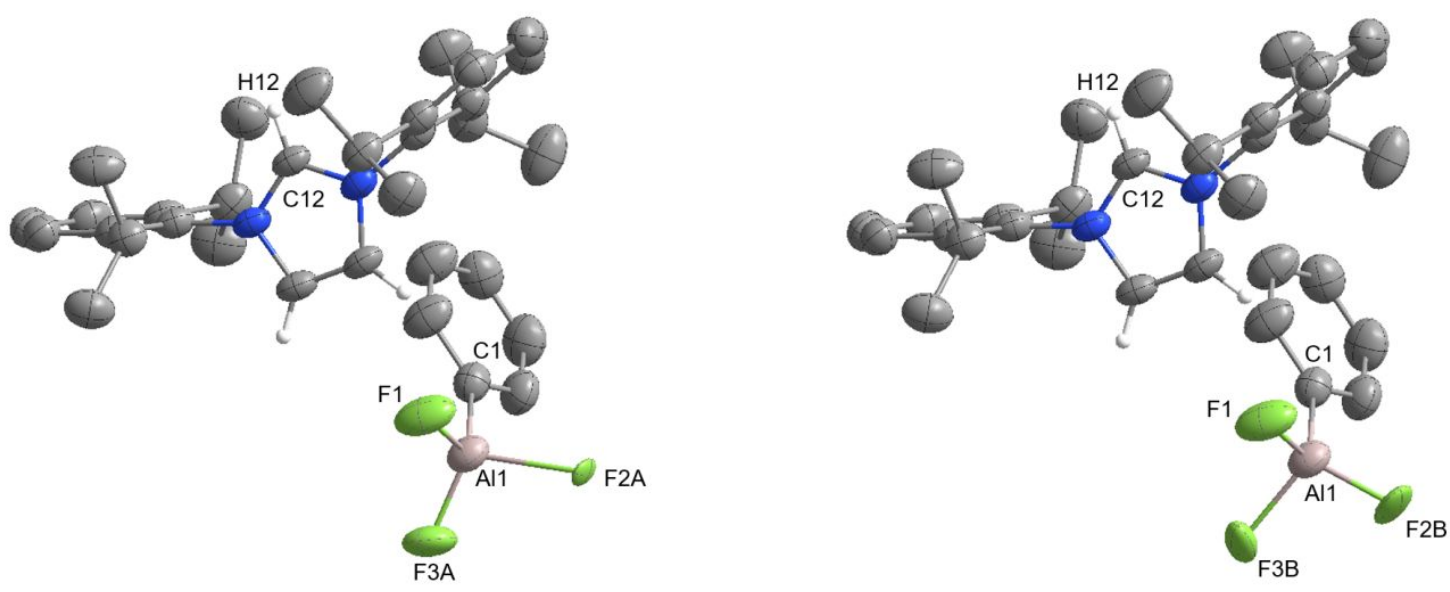

Figure S56. Crystal structure of the asymmetric unit of domain A (left) and domain B (right) of $[\mathrm{IPrH}]\left[\mathrm{PhAlF}_{3}\right](\mathbf{1 0})$. The ellipsoids are drawn at 50\% probability. For clarity, all hydrogen atoms are omitted except those on the imidazolium ring. 